\title{
Optimising the involvement of private practitioners in tuberculosis care and control in India
}

Citation for published version (APA):

Holalkere Yellappa, V. (2019). Optimising the involvement of private practitioners in tuberculosis care and control in India. [Doctoral Thesis, Maastricht University]. Maastricht University. https://doi.org/10.26481/dis.20191111vy

Document status and date:

Published: 11/11/2019

DOI:

10.26481/dis.20191111vy

Document Version:

Publisher's PDF, also known as Version of record

\section{Please check the document version of this publication:}

- A submitted manuscript is the version of the article upon submission and before peer-review. There can be important differences between the submitted version and the official published version of record.

People interested in the research are advised to contact the author for the final version of the publication, or visit the DOI to the publisher's website.

- The final author version and the galley proof are versions of the publication after peer review.

- The final published version features the final layout of the paper including the volume, issue and page numbers.

Link to publication

\footnotetext{
General rights rights.

- You may freely distribute the URL identifying the publication in the public portal. please follow below link for the End User Agreement:

www.umlib.nl/taverne-license

Take down policy

If you believe that this document breaches copyright please contact us at:

repository@maastrichtuniversity.nl

providing details and we will investigate your claim.
}

Copyright and moral rights for the publications made accessible in the public portal are retained by the authors and/or other copyright owners and it is a condition of accessing publications that users recognise and abide by the legal requirements associated with these

- Users may download and print one copy of any publication from the public portal for the purpose of private study or research.

- You may not further distribute the material or use it for any profit-making activity or commercial gain

If the publication is distributed under the terms of Article $25 \mathrm{fa}$ of the Dutch Copyright Act, indicated by the "Taverne" license above, 


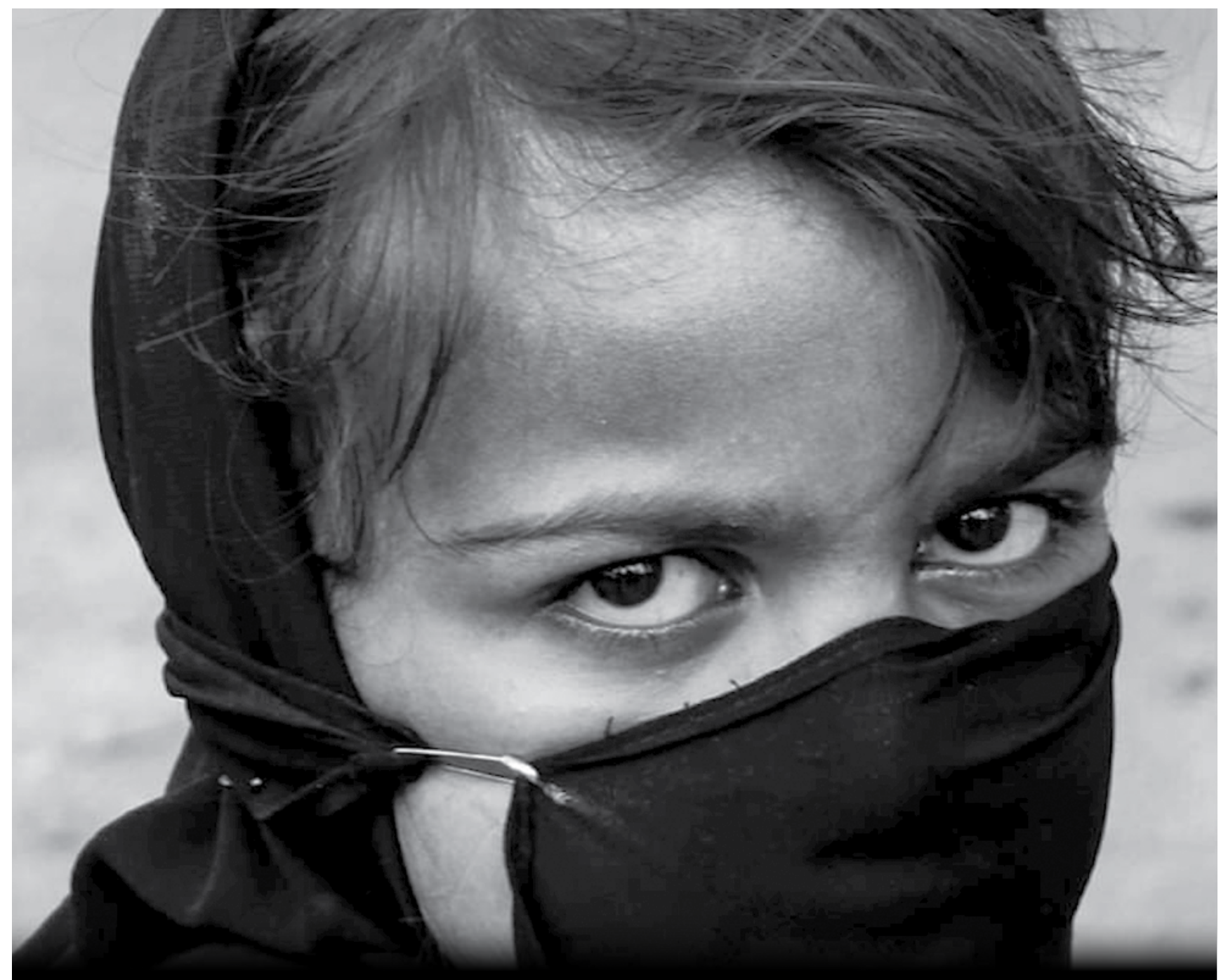

Optimising the Involvement of Private Practitioners in Guberculosis Care and Control in India

Dr. Vijayashree Yellappa 
Dissertation submitted to obtain the degree of Doctor at the Maastricht University, on the authority of the Rector Magnificus Prof.dr. Rianne M. Letschert in accordance with the decision of the Board of Deans

Maastricht University, Netherlands

Faculty of Health, Medicine and Life Sciences

Department of Health, Ethics and Society 
Optimising the Involvement of Private Practitioners in Tuberculosis Care and Control in India

\author{
Dr. Vijayashree Yellappa \\ Institute of Public Health \\ Bengaluru, India
}

Thesis submitted to fulfil the requirements of the doctoral degree at MAASTRICHT UNIVERSITY, NETHERLANDS 


\section{PROMOTERS}

Professor. Dr. Anja Krumeich (supervisor)

Maastricht University, Netherlands

Professor. Dr. Bart Criel(supervisor)

Institute of Tropical Medicine, Belgium

Dr. Narayanan Devadasan (co- supervisor)

Institute of Public Health, Bengaluru

Dr. Nora Engel (co- supervisor)

Maastricht University, Netherlands

\section{JURY}

\section{CHAIR}

Prof. Dr. B. van den Borne

Professor of Patient Information, Maastricht University

\section{MEMBERS}

Prof. Dr. C. Hoebe

Professor of Infectious Disease Control, Maastricht University

Prof. Dr. A. Venkat Raman

Professor of Human Resource Development and Health System, University of Delhi

Prof. Dr. W. Van Damme, Professor of Public Health, with focus on International Health Policies, Institute of Tropical Medicine, Antwerp, Belgium;

Dr. I. van der Putten Assistant Professor, Dept. of Health Service Research, Maastricht University. 


\section{I dedicate this thesis to my local PhD supervisor Dr N. Devadasan Director, Institute of Public Health, Bengaluru, India}

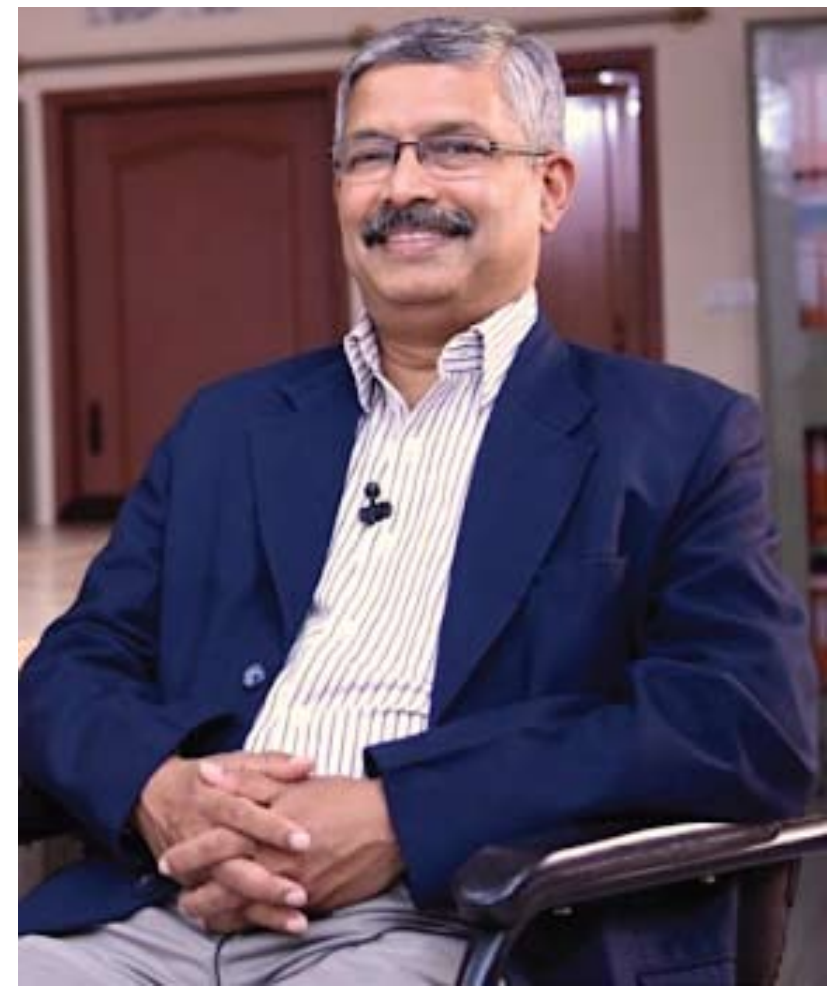

Deva, I will always be indebted for the guidance that I have received from you. I thank you for believing in my capabilities and encouraging me to do my best. As my mentor, you taught me more than I could ever give credit for. You have shown me, by your example, how a public health professional should conduct and contribute to the society. I dedicate this thesis and my $\mathrm{PhD}$ work to you. 



\section{TABLE OF CONTENTS}

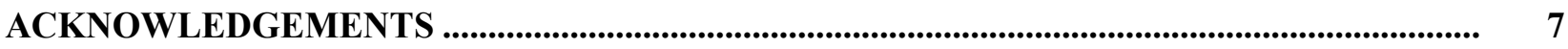

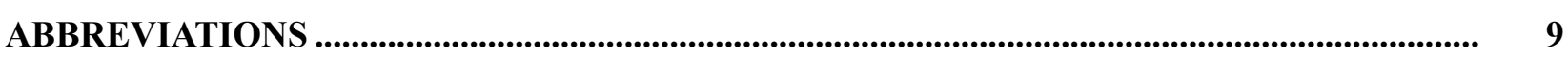

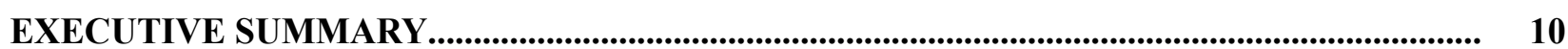

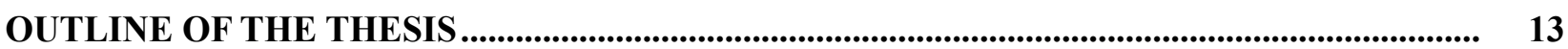

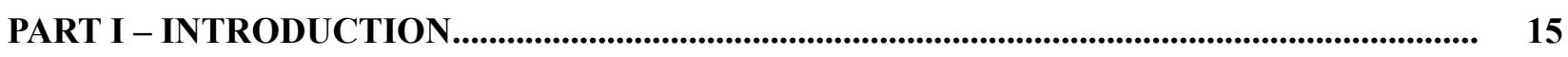

1. TUBERCULOSIS BURDEN AND THE RESPONSE ...................................................... 15

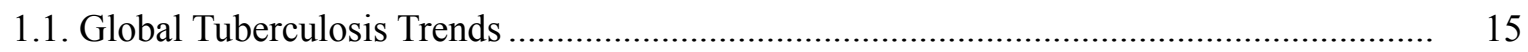

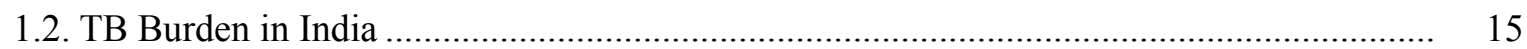

2. DOMINANT ROLE OF PPS IN THE PROVISION OF TB CARE AND CONTROL...... 17

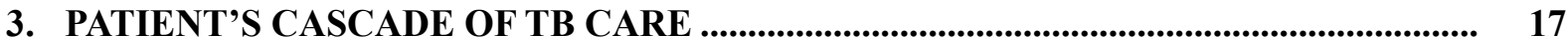

4. PUBLIC-PRIVATE MIX (PPM) IN TB CARE AND CONTROL....................................... 18

5. ASSESSMENT OF PUBLIC PRIVATE PARTNERSHIPS (PPPS) IN THE INDIAN HEALTH SYSTEM .............................................................................................................................. 20

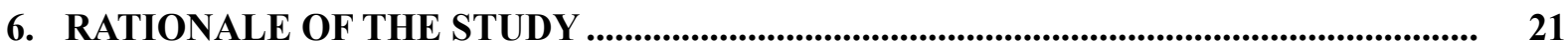

PART II - METHODS ......................................................................................................................... 23

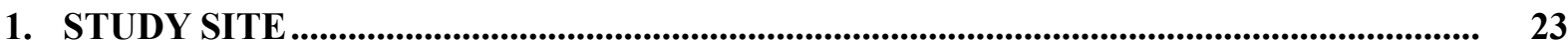

1.1. Profile of the District ......................................................................................................... 23

1.2. RNTCP in Tumkur District ..................................................................... 24

1.3. The Private Health Sector Landscape in Tumkur district............................................. 25

1.4. Administration and Implementation of PPM Schemes ..................................................... 26

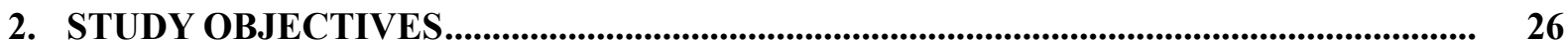

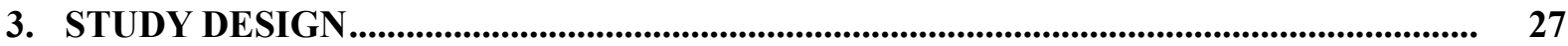

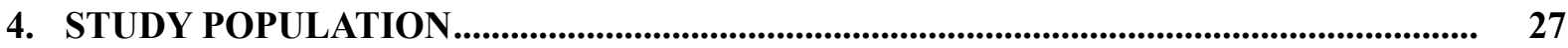

4.1. Inclusion and Exclusion Criteria ............................................................................................. 27

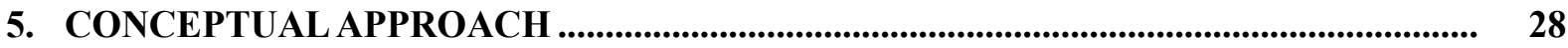

I. Pre-conditions phase (2012-2013)....................................................................... 28

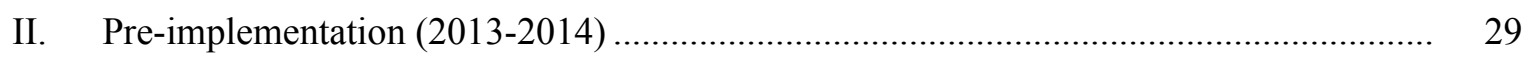

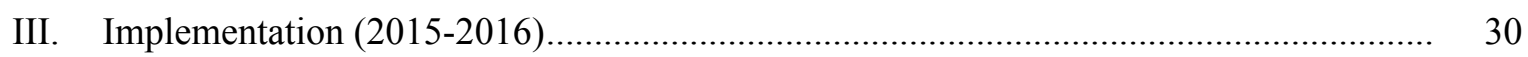

IV. Maintenance and Evolution (2016-2017) ..................................................................... $\quad 30$

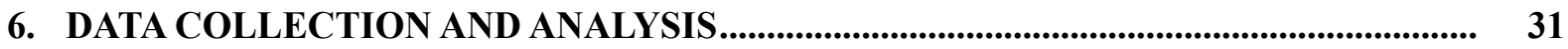

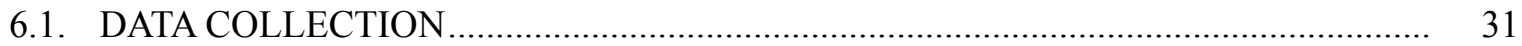

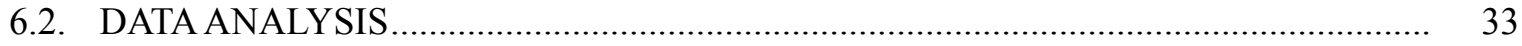

7. ETHICAL APPROVAL ................................................................................................................ 34

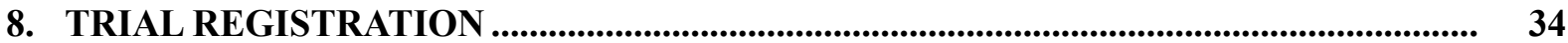




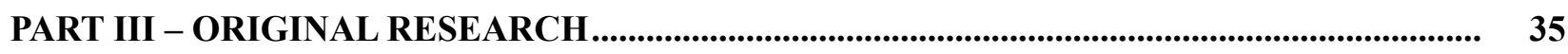

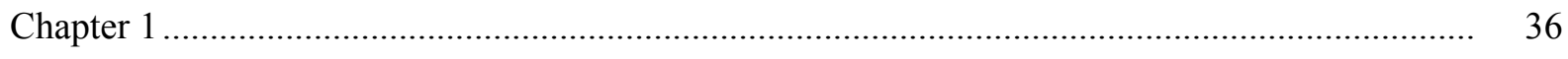

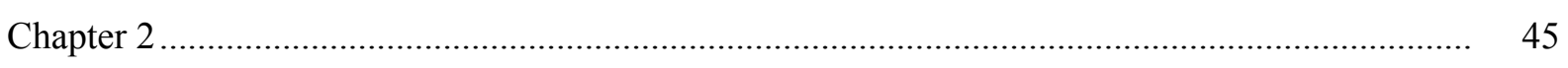

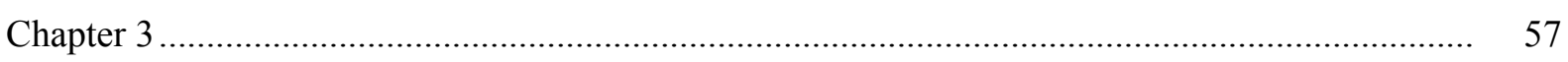

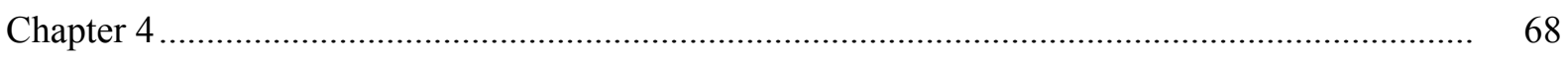

PART IV - GENERAL DISCUSSION_................................................................................. 79

1. ASSESSMENT OF LOCAL PRE-CONDITIONS FOR PARTNERSHIPS....................... 79

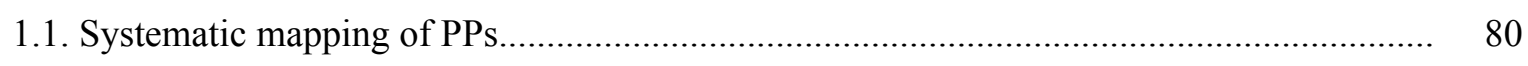

1.2. Recording and Reporting of Patient's Referrals from PPs to RNTCP ......................... 81

1.3. Patient's Therapeutic Itineraries to RNTCP \& PP's Cross Referral Practices ................ 82

2. TYPE OF PROVIDERS PRIORITISED FOR ENGAGEMENT .................................. 82

3. BUILDING PARTNERSHIP BETWEEN PPS \& RNTCP.............................................. 83

3.1. Designing the Intervention Package................................................................... 84

3.2. Training of PPs and the Key RNTCP Staff in PPM Strategy ...................................... 85

3.3. Building the Capacity of RNTCP Field Staff as An Interface of PPM Strategy ............ 86

3.4. Critical Need of Communication to Build Partnerships ............................................. 87

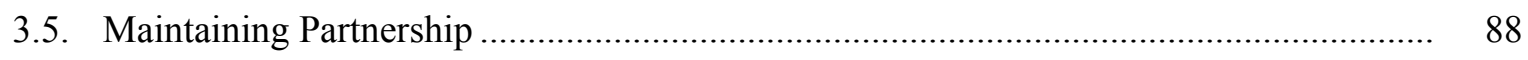

4. BUDGET ALLOCATION FOR PPM ACTIVITIES UNDER RNTCP............................ 89

5. PATIENT CENTERED CARE ...................................................................................... 89

6. STRENGTHENING GENERAL HEALTH SYSTEM FOR EFFECTIVE

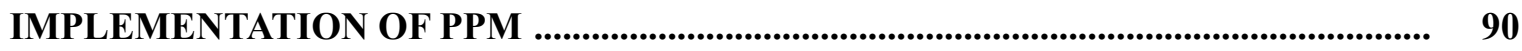

6.1. Local Health System Barriers to Engage PPs in RNTCP........................................ 91

6.2. Health System Regulations and Their Enforcement ................................................ 92

7. WAY FORWARD .................................................................................................. 93

7.1. Developing a Supportive Policy and Programmatic Environment .............................. 93

7.2. Moving from Pilots to Large-Scale Initiatives ...................................................... 95

7.3. Developing Monitoring and Evaluation Tools for PPM........................................... 96

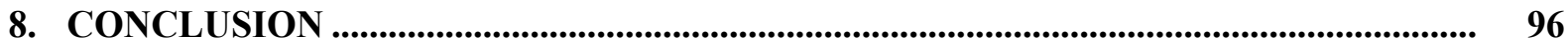

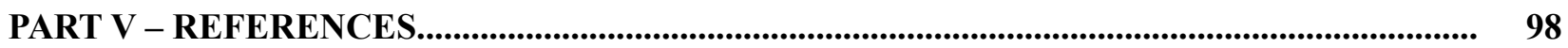




\section{ACKNOWLEDGEMENTS}

I have been supported by several people in my pursuit of knowledge spanning over six years. It was a period of intense learning, not only in the scientific arena, but also on a personal level.

The exposure I received at the Institute of Tropical Medicine, Belgium, one of the top public health schools of the world, was an incredible, impactful and pivotal one in my life. The educational training there had its own set of challenges, but every bit of it was worth the efforts. This work would not have been possible without the financial support that I received in the form of scholarship from the Director General of Development Corporation, Belgium Government.

I express my gratitude to my Ph.D. supervisor Professor Patrick Van Der Stuyft at Institute of Tropical Medicine. He taught me that 'science is a culture of doubt' and pushed me to think and see beyond what was evidently visible. I consider it as a great opportunity to do my doctoral programme under his guidance and learn from his vast research expertise. My special thanks to Pierre Lefèvre, who coached me in qualitative research methodologies. I also appreciate the assistance provided by Dr Tullia Battaglioli in carrying out complex quantitative data analysis and interpretation.

I would like to thank Professor Anja Krumeich from Maastricht University for her guidance and encouragement in completing my doctoral thesis. Special mention goes to my enthusiastic cosupervisor, Nora Engel. I thank her wholeheartedly for extending academic support and providing me with her concise insightful comments on the draft. I owe a lot to her for this achievement!

In this journey, I have not only gained research skills at the Institute of Tropical Medicine but had an opportunity to meet and interact with some of the most awesome people, who made a great impact on me. Firstly, I thank Professor Bart Criel, who was always there for me when I required help. His presence in the institute itself was enough for me to sail through this tough journey. I thank Ms. Greet Verhulst who received me with a smile whenever I landed in Belgium and made me feel at home. Fiona and others at the student service, were always there to answer my queries. I have to specially mention my friend Valeria Campos da Silveira for helping me remain calm. Roeland Scholtalbers, Head of Communications at Institute of Tropical Medicine, exposed me to the art of communication in public health. Thanks, Roe, for giving me an opportunity to work in the documentary movie and showcase my Ph.D. work.

Zamora and Marie who hosted me in Antwerp in 2014 and 2015, have become friends for life. I owe both of them a lot for their care and hospitality. 
I thank Dr. Sanath Kumar, District Tuberculosis Officer, Tumkur district, who discernably deserve the credit for success of this project. I thank field staff of Tumkur district for participating in this project, without whom this project would not have succeed the way it has today. I am indebted to the patients for thier time and for sharing their experiences. I thank private practitioners, especially Dr. Sudheer, Dr. Sandesh, and Dr. Muddurangappa and several others, who provided their valuable inputs.

I thank the team at Institue of Public Health, for all the support, especially my colleague Dr Dorothy who extended her support in a very special way. I thank Upendra and Prashanth for scholarly interactions at various points of my research programme. I thank my several friends, who kept cheering me up during the way.

I am indebted to my family, especially to my daughter Mrudula. She has been my greatest strength in this journey. We both embarked on a new academic journey in the same year, 2012 - she joined medical school and I enrolled for Ph.D. And now the time has come, when we both reach our destinations; she completes her degree to become a medical graduate and I complete my $\mathrm{PhD}$ journey.

Above all, I owe it all to Almighty God for giving me the wisdom, health and strength to undertake this research project and enabling me to see its completion. 


\section{ABBREVIATIONS}

AYUSH

Ayurveda, Yoga and Naturopathy, Unani, Sidda and Homeopathy

CTD

Central TB Division

$\mathrm{DMCs}$

Designated Microscopic Centers

DOTS

Directly Observed Treatment Short Course

DTO

District Tuberculosis Officer

FGD

Focus Group Discussions

GP

General Practitioners

MO

Medical Officer

MO-TB

Medical Officer -TB

$\mathrm{MoU}$

Memorandum of Understanding

NGO

Non-Governmental Organisations

PPP

Public Private Partnership

PPM

Public-Private Mix

PPs

Private Practitioners

RNTCP

Revised National Tuberculosis Control Programme

STO

State TB Officer

STS

Senior Treatment Supervisor

STLS

Senior Tuberculosis Laboratory Supervisor

TB

Tuberculosis

TUs

Tuberculosis Units

TB-HV

Tuberculosis-Health Visitor

WHO

World Health Organisation 


\section{EXECUTIVE SUMMARY}

Tuberculosis (TB) is the world's leading infectious disease, killing more people than any other infectious disease. India has the highest number of TB patients among the high TB burden countries in the world. The Government of India launched the Revised National TB Control Programme (RNTCP) in 1997 based on the World Health Organisation (WHO) endorsed Directly Observed Treatment, short course (DOTS). In spite of the availability of free diagnostic and treatment services at the public facilities, more than $50 \%$ of TB patients, irrespective of the income group, are estimated to seek care from private practitioners (PPs). Studies have demonstrated that PP's TB management practices in India are sub-optimal, and often TB is inaccurately diagnosed and ineffectively treated, thus posing a higher risk of mortality and drug resistance. Moreover, India is reported to have more than a million 'missing' TB patients every year that are not notified to the RNTCP. Thus, these patients do not get the standardised treatment.

Studies analysing the reasons for patient's diagnostic delay have concluded that seeking care from PPs in the initial period of illness was a clear risk factor. TB patients had to resort to multiple contacts with PPs because of PP's limitations in diagnosing and/or failure to direct patients to the RNTCP. Also, TB has resulted in a devastating socio-economic impact on patients from low-income households in the process of paying for inappropriate diagnostics and treatments. Though TB is a notifiable disease in India, TB patients diagnosed by PPs are often not notified to the RNTCP. Consequently, these patients are not included into the national data and mostly remain inadequately treated in the private sector.

Recognising the important role of PPs in the provision of TB care, the Indian Government is involving PPs in the RNTCP through the WHO recommended PPM (Public Private Mix) strategy since 2001. The PPM approach aims to strengthen TB care and control efforts through referral systems between the public and private providers and to provide standardised TB care. This partnership enables PPs to refer patients to the RNTCP for either free diagnosis and/or treatment. Thereafter, the patient can either continue treatment within the RNTCP or alternatively PPs themselves can provide DOT (Directly Observed Treatment) at their health facilities by procuring RNTCP drugs.

Several assessments of PPM initiatives in India have demonstrated that the PPM approach is feasible and effective. Yet, despite two decades of attempts to improve collaboration, PP's involvement in PPM strategy remains insignificant. Available published PPM studies have mostly focused on reporting the performance of PPM projects and little is known about the successful efforts to scale up PPM in TB control. The numbers demonstrating progress in PPM do not fully reflect the ground realities and questions remain as to how best to pursue sustainability and scalability of such initiatives. It is essential to recognise 'what works for whom, and under what conditions' to improve the understanding of 'how' partnerships with private sector providers work (or do not) by unpacking the dynamics between the different health system components. 
With this as the context, we designed a PPM model to optimise the involvement of for-profit allopathic PPs in RNTCP in a south Indian district. The study was conducted in four phases to improve referrals of presumptive pulmonary TB patients from PPs to RNTCP for sputum smear microscopy, and notification of TB patients to RNTCP who were either diagnosed and/or started on private TB treatment by PPs.

(i) Pre-conditions phase (2012-2013): We assessed the involvement of formal for-profit allopathic PPs in RNTCP in terms of referrals of presumptive pulmonary TB patients through review of RNTCP reports and registers.

(ii) Pre-implementation (2013-2014): In-depth interviews were conducted with stakeholders (PPs, TB patients, and RNTCP staff and managers) to understand the barriers for PPM implementation and PP's referred modes of collaboration. A systematic literature review was carried out simultaneously to identify effective interventions to engage PPs in TB care. After analysing participant's recommendations coupled with findings from the literature review, intervention package was designed to improve the collaboration between PPs and RNTCP.

(iii) Implementation phase (2014-2015): 316 PPs were identified through census and they were randomly allocated to an intervention and a control group. Intervention was implemented from December 2014 to January 2016, consisting of series of activities targeted towards key RNTCP staff and intervention PPs: (i) strengthening RNTCP staff capacity to collaborate with PPs; (ii) strengthening the documentation of TB patients referrals in laboratory registers; (iii) improving patient's accessibility to microscopic centres; (iv) counselling of patients by laboratory technicians to go back to the treating physician; (v) strengthening communication between the RNTCP and PPs through and (vi) training of PPs on PPM strategy and provision of TB related materials to PPs.

As the main outcome of the study, we assessed the proportion of PPs referring presumptive pulmonary TB patients to RNTCP and the proportion of PPs notifying TB patients started on private treatment to RNTCP in the intervention and control group. During the study period, PPs referred 836 patients and 176 were diagnosed with pulmonary TB. The proportion of referring PPs, mean referral rate per PP-year and smear-positive TB case-finding rate per PP-year were significantly higher in the intervention arm than the control arm. PP's referrals contributed to $20 \%$ of the sputumpositive pulmonary TB patients detected by RNTCP in Tumkur city (14\% were from intervention arm PPs).

(iv) Maintenance Phase (2016-2017): The positive findings from the study were integrated into the programme. During the project, the capacity of RNTCP staff was built to collaborate with PPs which enabled them to adapt the positive findings from the study. Further, PPM activities were integrated into the routine monitoring, which made the project self-sustainable. 
We demonstrated that a system-oriented intervention implemented by RNTCP staff based on the establishment of a strong referral and communication system with PPs, improved PP's referrals of presumptive pulmonary TB patients to RNTCP. Our study conducted in routine programmatic settings provides important information about the systemic impediments that affects engaging PPs in public health programmes. Nonetheless, replication warrants examination because the ability of a programme to transplant its model from one context to another context. Based on the outcomes of this study, intervention is extended to the control arm in the study area. Further, the model is rolled out and evaluated in rural parts of the same district. 


\section{OUTLINE OF THE THESIS}

The thesis aims to design, implement and test an intervention package to optimise the involvement of for-profit, allopathic private practitioners (PPs) in India's Revised National Tuberculosis (TB) Control Programme (RNTCP). This study is based on three sequential and interconnected sub-studies aimed at answering a series of specific research questions. The thesis comprises four major parts: Introduction, Methods, Results section consisting of original research papers, Discussion and Conclusion.

Part I, Introduction: It presents a review of the burden of TB globally and in India. Then it proceeds to describe the dominant role played by PPs in the provision of TB care and the challenges thereof, leading to complex therapeutic itineraries of TB patients. Following this, there is an overview of India's mixed health system dominated by the unregulated private health sector resulting in the poor quality of care. We then discuss about Public Private Partnerships (PPPs) which has emerged as an option to involve PPs in the public health programmes. Then we focus on RNTCP, a national health programme which engages PPs through Public Private Mix (PPM) strategy and discuss the status of PPM approach in India. The thesis then points to the development of rationale for the doctoral study, the concepts of PPM sustainability and scalability in India within the context of larger (PPP) in health care delivery.

Part II, Methods: This section describes the study setting, data collection and analysis, conceptual framework (Replicating Effective Programme-REP) that is used for the implementation of evidencebased interventions into community-based settings. This framework has four phases: pre-conditions (identifying need, target population and suitable intervention), pre-implementation (documentation and intervention packaging), implementation (package dissemination, training, technical assistance and evaluation), and maintenance and evolution (preparing the intervention for sustainability). In this section, we discuss how research activities were designed in each phase, how they were related to each other and how they collectively attempt to answer the research questions.

Part III, Results: This section provides research findings from the different phases of study in a chronological order:

Chapter 1 sheds light on the situational analysis of PP's participation in the PPM activities of RNTCP and the pre-conditions available for PPM in the study area.

Chapters II and III describe TB patient's experiences of completing treatment in RNTCP and their complex itineraries to TB care respectively. The latter chapter also sheds light on PP's TB management practices and their preferred modes of collaboration with RNTCP. These chapters form a part of the pre-implementation phase and guided designing of intervention package to involve PPs in RNTCP. 
Chapter IV demonstrates the implementation and the effect of intervention package, and the health system challenges in building a partnership between private and public counterparts.

Part IV, General Discussion and Conclusion: It brings together the findings of the research study and discusses them in relation to the existing literature. While making specific recommendations, it also outlines areas that require further research in order to better understand and frame the health systems' response to TB control activities by engaging PPs and PPPs in health broadly. 


\section{PART I - INTRODUCTION}

\section{TUBERCULOSIS BURDEN AND THE RESPONSE}

\subsection{Global Tuberculosis Trends}

Tuberculosis (TB) is the world's leading infectious disease, killing more people than any other infectious disease. Globally, an estimated 10.4 million people fell ill and an estimated 1.3 million people died of TB in 2016 (Organisation 2017). Around 45\% of these estimated number of new TB patients were reported in the South-East Asia Region (Organisation 2017). In 1993, World Health Organisation (WHO) declared TB as a global emergency (World health organisation 1994). Since then, there have been concerted global efforts to address TB as a global health problem. Millions of people are diagnosed and successfully treated for TB each year, averting millions of deaths (53 million in the period 2000-2016). However, there are still large gaps in the timely detection of TB and successful treatment completion (Organisation 2017). It is estimated that 3 million patients a year still go undetected and/or unreported. Many of these missing patients are likely to be in the private sector (World Health Organization 2016). Globally, systematic efforts to engage the Private Practitioners (PPs) in TB control have been underway for the past two decades. These efforts under the 'Public-Private Mix (PPM)' strategy aim to strengthen TB care and management efforts through referral systems between the public and the private providers, to reach out to more patients and to provide standardised diagnosis and treatment (WHO 2006). Central to the concept of PPM is the identification of the 'mix' of clinical tasks (e.g. identifying and referring people with TB symptoms, making a diagnosis, prescribing treatment) and public health tasks (e.g. undertaking laboratory quality assurance, supporting patient follow-up, recording and reporting data) that are essential for the delivery of quality TB care (M Uplekar 2016).

\subsection{TB Burden in India}

India accounts for about a quarter of the world's total TB patients and it remains a major public health problem in India. The number of new TB patients per year is estimated to be 2.8 million (Organisation 2017) and an estimated 480,000 patients die every year (Central TB Division, Directorate General of Health Services 2017). According to the Registrar General of India's Millions Death study, which documented causes of death in 1.4 million households, TB remained one of the top five causes of death among people aged 30-69 years (Aleksandrowicz et al. 2014). The report released by the Department of Health Research, Government of India, further reported TB as the leading cause 
among the infectious diseases (Indian Council of Medical Research, Public Health Foundation of India 2017). The Government of India launched the Revised National TB Control Programme (RNTCP) in 1997 based on the WHO-endorsed Directly Observed Treatment Short Course (DOTS) (Sachdeva et al. 2012). The targets have been set to detect at least $70 \%$ of the estimated new sputum smear-positive pulmonary TB patients and cure at least $85 \%$ of all newly detected pulmonary TB patients (Central TB Division; Government of India; 2017). The programme follows a 'passive case finding' approach, which assumes that people are able to recognise their symptoms and seek health care. Patients can avail RNTCP services free of cost either by directly accessing public health facilities or by being referred by private practitioners (PPs) to RNTCP.

India has done well to rapidly expand RNTCP services to cover its huge population by March 2006. The incidence of TB has reduced from 289 per lakh per year in 2000 to 217 per lakh per year in 2015, and the mortality due to TB has reduced from 56 per 100,000 per year in 2000 to 36 per 100,000 per year in 2015 (Central TB Division; Government of India; 2017). However, in spite of these efforts, the burden of TB in India continues to remain high (Central TB Division 2018). Going by the current rate of decline, India is far from reaching the goals of End TB strategy, which has goals of a 95\% reduction in TB deaths (compared with 2015), a 90\% reduction in TB incidence (10 patients per 100000 population) by the year 2035 (Mukund Uplekar et al. 2015). Further, the Government of India has announced a goal to achieve a rapid decline in the burden of $\mathrm{TB}$, morbidity and mortality, while working towards the elimination of TB in India by 2025 in its National Strategic Plan for TB elimination 2017-2015 (Central TB Division, Directorate General of Health Services 2017). But the ground reality is that India tops the list of 10 countries that account for $74 \%$ (2.4 million) of the estimated 'missed cases' globally (World Health Organization 2016). 'Missed cases' are those patients which are neither detected nor notified to RNTCP. This can be attributed to either patients delay in accessing healthcare or health system delays (Veesa et al. 2018), (Mistry et al. 2017) or to PP's reluctance to notify patients to RNTCP (Geeta Pardeshi, Andrea Deluca, Sutapa Agarwal 2018), (Philip et al. 2015), (Thomas et al. 2016). Providing universal access to early diagnosis and treatment and improving case detection were the main goals of the national strategic plan 201217 (central TB Division, Directorate general of health services 2012). But RNTCP failed on both counts, as pointed out by the Joint Monitoring Mission report of 2015 (World Health Organisation 2016). Further, the use of non-standard diagnosis and TB treatment protocols by PPs remain a huge challenge in India (World Health Organisation 2016). Mostly the TB patients are diagnosed only after several months of seeing multiple providers, since most of PPs do not follow standard treatment guidelines (Cazabon et al. 2017), (Das et al. 2015), (S Satyanarayana et al. 2015). Apart from the poor quality of care, TB in India is fuelled by broader socio-economic factors, including extreme poverty, high prevalence of malnutrition, smoking and diabetes (Bishwajit, Ide, and Ghosh 2014). 


\section{DOMINANT ROLE OF PPS IN THE PROVISION OF TB CARE AND CONTROL}

In India RNTCP is implemented mainly through public sector health facilities, which provide free diagnostic and treatment services. In spite of the availability of free public services, more than $50 \%$ patients, irrespective of the income group, are estimated to seek care in the private health sector, because of the perceived good quality of care as compared to the public sector (Srinath Satyanarayana, Nair, Chadha, Shivashankar, Sharma, Yadav, Mohanty, Kamineni, Charles, et al. 2011), (Hazarika 2011), (Nimbarte, Wagh, and Selokar 2011), (Mistry et al. 2017), (Kapoor, Raman, Sachdeva, \& Satyanarayana, 2012). A study which documented the enormous quantities of anti-TB drugs that are used in the private sector, underscored the importance of PPs in the provision of TB care (Arinaminpathy et al. 2016). Recognizing the important role of PPs in TB care, the government is trying to involve PPs in RNTCP through WHO recommended PPM (Public Private Mix) strategy (WHO 2010). This partnership enables PPs to refer patients to RNTCP for either free diagnosis or treatment. Thereafter, the patient can either continue treatment within RNTCP or alternatively PPs themselves can provide DOTS (Central TB Division, Directorate general of health services 2014).

Best practices for TB diagnosis and treatment are enshrined in the Indian Standards of TB Care (Organisation 2014). However, PP's rarely follow standard TB management guidelines in India (Achanta et al. 2013). Studies have shown that PP's TB management practices are sub-optimal, where TB is often inaccurately diagnosed and ineffectively treated (S Satyanarayana et al. 2015), (Udwadia, Pinto, and Uplekar 2010a), (C T Sreeramareddy et al. 2014a), (Anand et al. 2017), (Jarosawlski S 2012), (Pai, Yadav, and Anupindi 2014), (Bhargava, Pinto, and Pai 2011). Supporting this fact, a systematic review has shown that seeking initial care from a PP is clearly a significant risk factor for diagnostic delay (Chandrashekhar T Sreeramareddy et al. 2014), (Mishra and Mulani 2013). Uplekar, et al. in his study (Uplekar, Mukund, S. Juvekar, S. Morankar, S. Rangan 1998) showed that many PPs referred patients to government hospitals only late in the course of the patient's illness and consequently making patient itineraries complex. These study findings make a compelling case to engage PPs in TB care to shorten a patient's care-seeking pathway. Further, TB patients that are treated by PPs are neither notified nor their treatment outcomes recorded. Therefore, these patients are not included into the national data and they mostly remain inadequately treated in the private sector (Subbaraman et al. 2016). A model projected that if these practices are not corrected over the next 20 years, (Law et al. 2017) the TB epidemic will shift gradually from one that is predominantly drug susceptible to one with increasing drug resistance.

\section{PATIENT'S CASCADE OF TB CARE}

RNTCP is implemented mainly through government health facilities, which provide quality assured free diagnosis and treatment. Currently, RNTCP follows the 'passive case finding approach', which assumes that people are able to recognise their symptoms and access health care in time. PPs are 
often the first choice for seeking TB care in India (Satyanarayana et al., 2011), (C T Sreeramareddy et al. 2014a), before they turn to public institutions (Kapoor, Raman, Sachdeva, \& Satyanarayana, 2012), (Anand et al. 2017), (Mistry et al. 2017). This is because of PP's ability to provide greater ease of access, shorter waiting periods, more flexible opening hours, and better availability of staff. But PPs in India rarely follow standard TB management guidelines (Jarosawlski S 2012), (Achanta et al. 2013), (S Satyanarayana et al. 2015), (C T Sreeramareddy et al. 2014b) and thier widespread empirical management and poor quality of care in India has led to poor treatment outcomes (McDowell and Pai 2016), (Das et al. 2015). Further, because of the provider's inability to diagnose the disease, or failure to direct the patients to RNTCP, patients are forced to concurrently and/or iteratively seek care from public and private providers, causing important diagnostic and treatment delays leading to long care-seeking pathways (Mistry et al. 2017), (Kapoor et al. 2012), (Nimbarte, Wagh, and Selokar 2011), (Srinath Satyanarayana et al. 2015), (Jaggarajamma et al. 2009), (Charles et al. 2010), (Udwadia, Pinto, and Uplekar 2010b). The Stop TB progress report on 90-90-90 (STOP TB Partnership 2017) targets shows a similar trend, where only 1 in 2 patients with drug-sensitive TB gets adequate diagnosis and treatment, while 1 in 5 patients with drug-resistant TB gets adequate care.

These broken TB care cascades have disastrous consequences for patients especially from lowincome households (Veesa et al. 2018), who tend to lose several months of their income in the process of paying for inappropriate diagnostics and treatments before starting therapy under RNTCP (Tanimura et al. 2014), (Pantoja, Floyd, et al. 2009a), (John et al. 2009), (Floyd et al. 2006), (Nimbarte, Wagh, and Selokar 2011). When patients finally reach the public sector health facilities, they are financially constrained, and in many cases, they are found to have developed drug-resistant TB (Udwadia 2012), (Charles et al. 2010). These results demonstrate the importance of private sector engagement in TB care and management to shorten the care-seeking pathway. Further, TB advocates have been urging for a patient-centric solution for TB control delivered with dignity and compassion (Reid and Goosby 2017), (Pai, Yadav, and Anupindi 2014). This requires a meaningful engagement of PPs with RNTCP, with due attention to patient's needs and expectations from RNTCP. Currently, systematic efforts in this direction seem to be inadequate.

\section{PUBLIC-PRIVATE MIX (PPM) IN TB CARE AND CONTROL}

The Indian government involves PPs through the WHO recommended PPM strategy (WHO 2010). The RNTCP has designed a set of guidelines to develop partnership and to effectively link RNTCP with all public and private healthcare providers so as to detect and provide standardised treatment to TB patients (Central TB Division, Directorate general of health services 2014). Currently, there are four broader options for engagement, such as advocacy communication and social mobilisation, diagnosis and treatment, managing TB and comorbidities and programme management option. The PPM guidelines provide different partnership options for engagement of NGOs and PPs, principally based on results-based financing. Each scheme has specific objectives that the partnership is expected 
to fulfil by signing a memorandum of understanding with the district TB officer, depending on their managerial and technical capacity (Neeraj Kulshrestha a, Sreenivas Achuthan Nair b, K. Rade c, A. Moitra c, P. Diwand 2015).

The Government of India's efforts of to implement PPM-TB strategy date back to the period 19972003. During this period, PPM models were piloted in many places in the country such as Delhi (Arora, Sarin, and Lönnroth 2003), Hyderabad (Murthy et al. 2001), Kannur (Granich and Chauhan 2005), Mumbai (Ambe, Lönnroth, Dholakia, Copreaux, Zignol, Borremans, and Uplekar 2005), and tea garden hospitals in North East India (Agarwal, S P 2005). Using the positive experiences gained from these collaborations, RNTCP published formal PPM guidelines to structure collaborations between RNTCP and an NGO (central TB Division, Directorate general of health services 2001), and PPs (Division, Central TB,directorate general of health services 2000). These guidelines were subsequently revised in 2008 (Central TB Division 2008) and 2014 (Central TB Division, Directorate general of health services 2014). Additionally, the Standards for TB Care in India published in 2014 (Organisation 2014) lays down the uniform standards for TB care for all stakeholders in the country. Apart from these PPM schemes, any PPP, community health worker or social worker can become a DOT provider without formally getting engaged with RNTCP. DOT providers are given a financial incentive of INR 500 when a TB patient completes RNTCP treatment and INR 2000 for multi-drug resistance TB cases (Tb et al., n.d.). In parallel, the Government of India undertook certain policy initiatives in RNTCP to improve the engagement with PPs. TB has been classified as a notifiable disease from 2012 making it mandatory for all private health establishments (laboratories, allopathic hospitals/clinics/nursing homes and individual allopathic PPs) to register with RNTCP for notifying TB patients diagnosed and/or treated by them to the government authorities ( Ministry of Health and Family Welfare, Government of India 2012). NIKSHAY, a web-enabled application was launched in 2012 with an objective to create a database of all TB patients across the country (M. of H. \& F. W. Government of India 2015). Details of TB patients can be notified through hardcopy, email, phone call or SMS, or can be directly uploaded on NIKSHAY. Besides the improvement in TB surveillance, RNTCP sees the notification as an opportunity to extend the support mechanisms for ensuring treatment adherence and its subsequent completion, especially for TB patients treated by PPs (Sachdeva et al. 2012).

Despite two decades of efforts to improve collaboration between PPs and RNTCP, it has not yielded desired results (Sachdeva et al. 2012). Assessments of RNTCP-PPM initiatives in India have demonstrated that the PPM approach is feasible and effective in achieving the improved case detection as well as adequate levels of treatment outcomes (Dewan et al. 2006), (Granich and Chauhan 2005), (Ambe, Lönnroth, Dholakia, Copreaux, Zignol, Borremans, Uplekar, et al. 2005, Kumar et al. 2005; Murthy et al. 2001), (Lal, Sahu, et al. 2011b), (Ambe, Lönnroth, Dholakia, Copreaux, Zignol, Borremans, Uplekar, et al. 2005; Murthy et al. 2001; Floyd et al. 2006; Kumar et al. 2005), (Arora, Sarin, and Lönnroth 2003), (Balasubramanian et al. 2006). Economic analysis of the PPM strategy has further suggested that such collaborations are cost-effective interventions (Floyd et al. 2006), 
(Pantoja, Floyd, et al. 2009b), (Ferroussier et al. 2007). However, lessons from these successful experiences have not been pursued or scaled up across the country. The studies cited above have focused merely on performance indicators (Baloch and Pai 2012), and have not paid enough attention to the implementation problems of PPM activities. Moreover, the numbers demonstrating progress in PPM do not fully reflect the ground realities (Udwadia, Pinto, and Uplekar 2010c) and questions remain as how to best pursue sustainability and scalability with such initiatives.

\section{ASSESSMENT OF PUBLIC PRIVATE PARTNERSHIPS (PPPs) IN THE INDIAN HEALTH SYSTEM}

When we focus on the scope of broader PPPs in healthcare in India, use of PPPs has grown almost five-fold worldwide (PwC Health Research Institution 2010). Further, different types of partnerships have been developed based on the purpose, outcomes, and partners involved in partnerships (Widdus, 2005), (Berlan \& Shiffman, 2012), (Basu et al. 2012). However, experiences in PPP is still limited and poorly documented (Government of India 2012). Researchers have opined that PPPs in developing countries suffer from conceptual imprecision and that the debate is often rich in opinions and short on facts (Nishtar, 2004), (Gajda, 2004), (Reich, 2000), and they do not inform us about under 'what' conditions they work successfully (Nishtar 2007), (Buse \& Tanaka, 2011). Baru and Nundy (Baru and Nundy 2008), who studied PPPs in healthcare reasoned that PPPs have been built without organisational and administrative preparedness, raising questions regarding their role, accountability and effectiveness. Reiterating this fact, Barbara McPake, while comparing international health sector reforms, reported that India never had a national policy for PPPs and that the arrangements were established on an ad hoc basis (McPake and Mills 2000). Others reported shortcomings of PPPs such as lack of accountability, non-transparent mechanisms and lack of stakeholder consultation (Annigeri, Vinod B, Prosser Lizann and Roy 2004), (P. C. Government of India 2004).

The Indian government's task force (Planning Commsion 2012) which assessed the performance of PPPs in health sector reported that the existing evidence for PPPs in health sector does not allow for easy generalisation and PPPs are not documented well enough to understand the PPP implementation processes. Additionally, it reported that the success of PPPs was mostly dependent on charismatic individuals who managed the PPPs. Supporting this fact, other authors have debated that the success of complex partnerships depends heavily on the relationship between the actors and how they adjust with each other over time and space in a larger health system (Thomason \& Rodney, 2009), (Buse \& Harmer, 2004). It is also reasoned that if the success of an PPP intervention is overly dependent on a key individual, long-term sustainability of the partnership is mostly unreliable (Venkat Raman 2008). Hence it is essential to recognise 'what works for whom, and under what conditions' to improve our understanding of 'how' partnerships with private sector providers work (or does not), (Buse \& Waxman, 2001), (Quelin 2012), (Jens K. Roehrich, Michael A. Lewis 2014), by unpacking the dynamics between the different health system components (Jamali, 2004), (Kapilashrami, 2010). 


\section{RATIONALE OF THE STUDY}

The above cited evidence holds good for the PPM approach in TB care. Despite two decades of efforts to improve collaboration between PPs and RNTCP, the results have not been desirable (Sachdeva et al. 2012). Notwithstanding the promises shown, the PPM strategies have not been scaled up and the national coverage by these models has often not been successful. The evidence and literature fails to account for the variations that exist in the designs across states and rural/ urban areas, and the processes of building these partnerships (Kumar et al. 2005), (Dewan et al. 2006), (M Uplekar 2016). Moreover, the documented examples of successful collaboration on the ground are few and there are certain unknowns which persist to sustainably engage PPs in RNTCP (M. S. Khan, Salve, and Porter 2015). Several challenges remain in the implementation of PPM, in terms of distrust between private and public providers (Ayesha De Costa, Eva Johansson 2008), (Vyas, Small, and Deriemer 2003), lack of training and information, as well as poor incentives and regulatory mechanisms in implementing the PPM (Uplekar et al., 2001a).

Notwithstanding the promises shown, the PPM strategies have not been scaled up and the national coverage by these models has often not been successful. The evidence and literature fail to account for the variations that exist in the designs across states and rural/urban areas, and also the processes of building these partnerships (Kumar et al. 2005, Dewan et al. 2006, Uplekar et al. 2001). Moreover, the documented examples of successful collaboration on the ground are few and there are certain unknowns which persist to sustainably engage PPs in RNTCP (M. S. Khan, Salve, and Porter 2015). Several challenges remain in the implementation of PPM, in terms of distrust between private and public providers (De Costa, Johansson, \& Diwan, 2007; Vyas, Small, \& DeRiemer, 2003), lack of training and information, as well as poor incentives and regulatory mechanisms in implementing the PPM (Uplekar et al., 2001a). A study on PPM in TB control in India as a case of organisational innovation Engel, et al. (Engel and Bijker 2012) reported that resistance and apprehensions against PPM and the external partners continue at the field level, by district- and subdistrict-level RNTCP staff. The different PPM initiatives are built around different problem definitions of what PPM should solve and different control practices, including supervision, standardisation and work culture, which continue to clash and ultimately hinder the scaling up of PPM (Engel and van Lente 2014).

There are arguments that the PPM strategy is based on a meagre evidence base (Mahendradhata et al. 2007a) and that the success of the PPM strategy depends on the capacity of the general health systems within which they are delivered (Lei et al. 2015), (Mahendradhata et al. 2003), (Lönnroth et al. 2004), (Unger, J.P., De Paepe, P., Ghilbert 2010) and how the processes of collaboration are managed (Probandari A, Utarini A, Lindholm L 2011). There are important gaps in the scholarly and practitioner's understanding of how the concept has been applied at the ground level. Evidence, though limited, underscores the need for careful assessment of PPM arrangements, the role of the partner organisations in relation to the State, the programme and the health system in general. 
Further, understanding the potential for replication and the modalities for scaling up the successful approaches is important. However, it currently remains unclear within health systems.

In this context, we developed a PPM model to optimise the involvement of for-profit allopathic PPs in RNTCP and assessed the effectiveness of the model. This study has been designed within the broader scope of the National Strategic Plan of RNTCP (2012-2017), (central TB Division,Directorate general of health services 2012). We believed that the PPM approach is really a form of behaviour change for both sides: for the public sector in their approach towards engaging the private sector and for the private sector in changing their TB management practices. Therefore, the model focused on strengthening RNTCP functioning to collaborate with PPs and streamlining the organisational mechanisms to improve referrals of presumptive pulmonary TB patients and notification of TB patients from PPs to RNTCP. This study is timely and important, because there is stress on the goal to improve the sustainability of private sector engagement to end the TB epidemic by 2025 in the National Strategic Plan for TB Elimination (Central TB Division, Directorate General of Health Services 2017) to provide universal access to quality TB diagnosis and treatment. This study, conducted in routine programmatic settings, provides evidence supporting the use of low-cost interventions built within national TB programme to increase PP's involvement in the programme. 


\section{PART II - METHODS}

\section{STUDY SITE}

\subsection{Profile of the District}

The study was carried out in Tumkur city (population 384,016), the district headquarters of Tumkur district (population $\sim 28$ million), situated $70 \mathrm{~km}$ North West of Bangalore, the capital of Karnataka state. The district has 10 sub-districts. See Figures 1,2 and 3 for the location of Karnataka state in the map of India, the location of Tumkur district in Karnataka state and Tumkur district respectively.

Like everywhere in India, Tumkur district also has a free government healthcare service. There are three tiers of healthcare providers based on the Indian Public Health Standards (Directorate General of Health Services Ministry of Health \& Family Welfare and India 2012). Figure 4 shows the structure. Each primary health centre carries out outreach activities through health sub-centres, wherein auxiliary nurses are in charge of providing antenatal care, immunising children and detecting communicable diseases at an early stage.

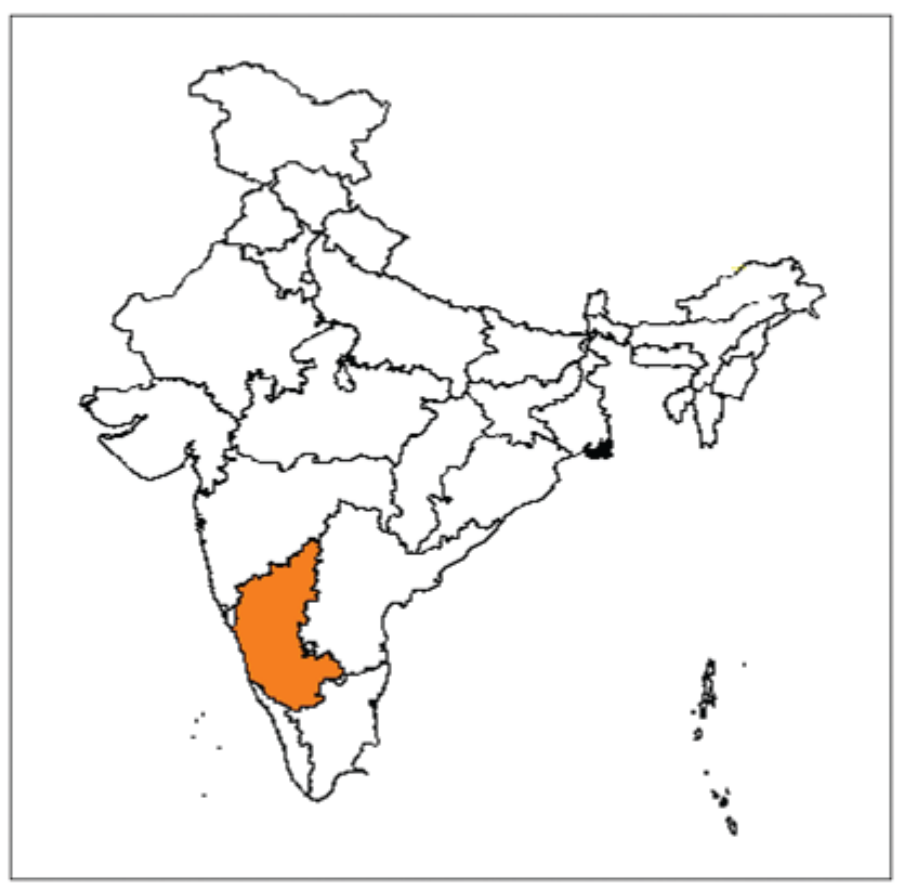

Figure 1: Map of India showing Karnataka province in colour

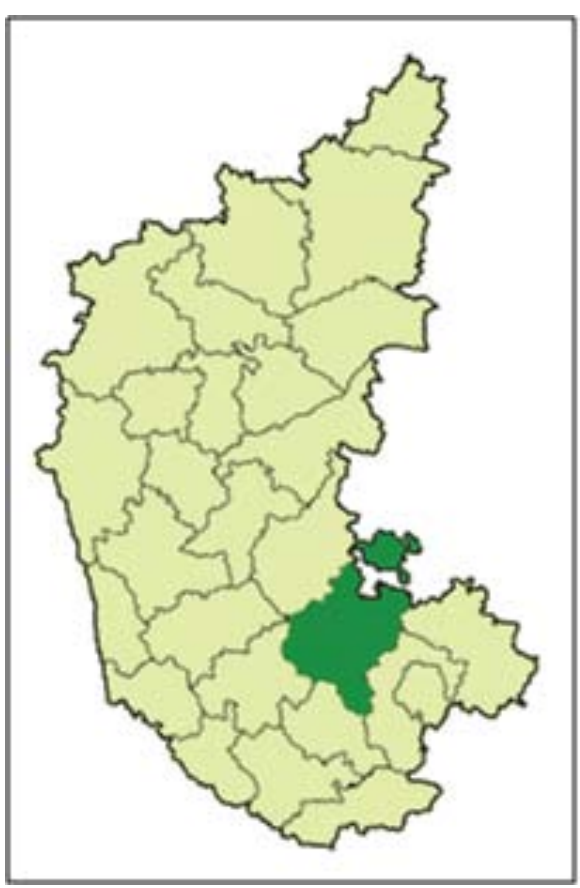

Figure 2: Location of Tumkur district in Karnataka province 


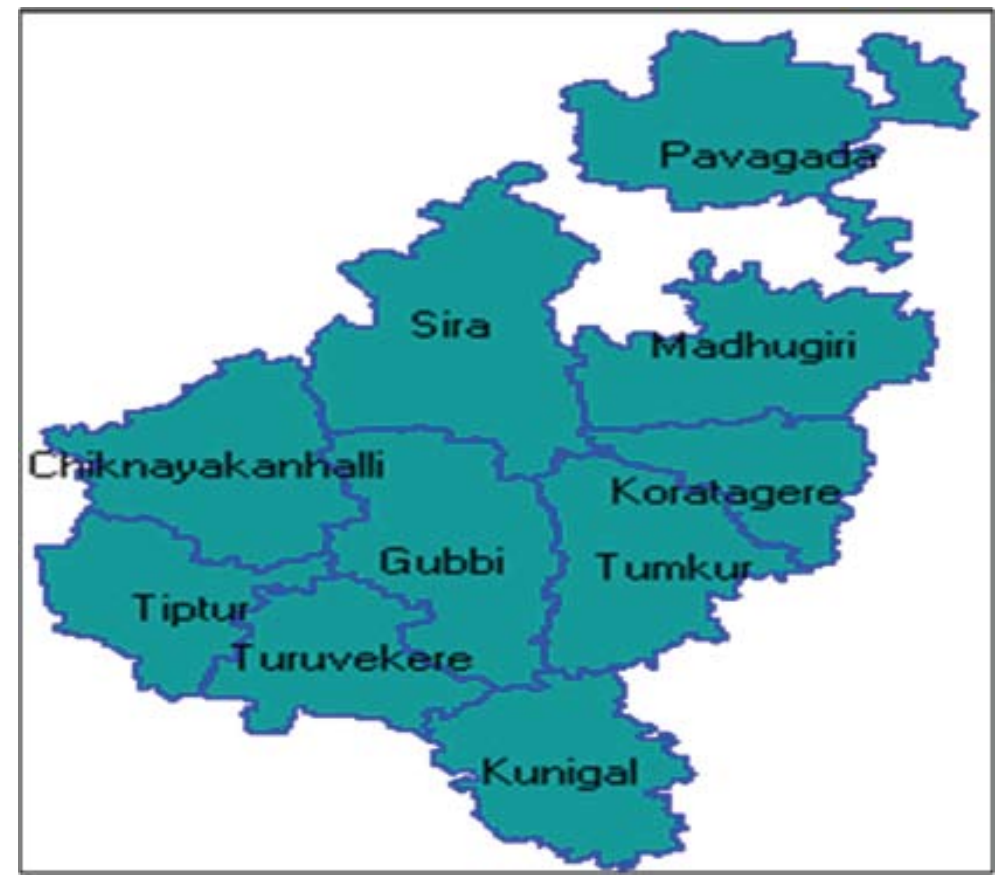

Figure 3: Map of Tumkur district

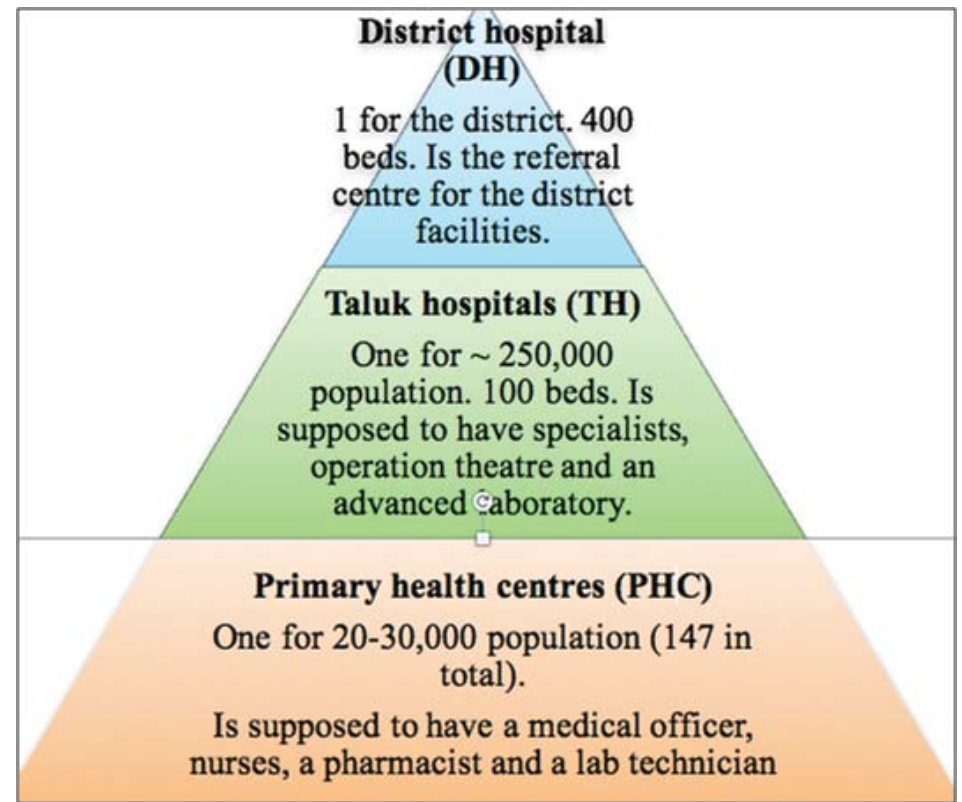

Figure 4: The Government Health Service Organisation in Tumkur in 2013

\subsection{RNTCP in Tumkur District}

RNTCP was launched in Tumkur district in 2005-2006. In order to receive free RNTCP services, patients can either directly access any of these government health facilities or can be referred by PPs. As per RNTCP guidelines, Tumkur district is divided into 10 tuberculosis units (TUs), aligned with the administrative sub-districts. TU is responsible for the maintenance of TB registers and 
the timely submission of reports to the district level. Each TU is manned by a senior treatment supervisor (STS) who is in charge of TB treatment, a senior TB laboratory supervisor (STLS) who is in charge of sputum microscopy activities at microscopic centres and a medical officer for TB Control to oversee RNTCP activities in the TU. In urban areas, one TB health visitor (TB-HV) is present for every 100,000 population to oversee patient treatment adherence and provide health education ( $\mathrm{n}=4$ in Tumkur city).

Further each TU is divided into five designated microscopic centres, each catering to a population of 100,000. Tumkur city has three microscopic centres (one each at the district TB centre, district hospital and Siddhartha Medical College). The district TB officer is the head of the programme, stationed at the district TB centre. There are 2,555 DOT centres that provide free TB drugs and motivate patients for treatment completion. DOTS provider can be either a staff of a public health facility (all of them provide DOTS), a PP, a health worker or any trained community volunteer, who is acceptable to and accessible by patients and accountable to the health system. There are five urban primary health centres in Tumkur city. All of them collect sputum samples and transport them to the nearest microscopic centres.

\subsection{The Private Health Sector Landscape in Tumkur district}

There is a dominant private health sector with a wide spectrum of healthcare providers, ranging from unqualified practitioners to highly trained specialists. For our study, PPs were defined as 'formal' if they were formally trained either in allopathic medicine or in the Indian system of medicine Ayurveda, Yoga and Naturopathy, Unani, Sidda and Homeopathy (AYUSH). PPs who were trained in AYUSH, but practised allopathy was defined as 'informal'. Allopathic PPs with advanced training were categorised as 'specialists' and others with no specialist training were classified as 'general practitioners (GPs)'. Box 1 provides definitions of the different types of PPs:

\section{Box 1: Definitions of type of PPs}

System of Medicine: PPs are broadly categorised based on the training.

Allopathic: Bachelor of allopathic medicine

AYUSH: Bachelor of Indian system of medicine

Informal: No training whatsoever

Type of specialty: Allopathic PPs are categorised based on whether they have pursued any advanced training. They are categorised as general practitioners (GPs) if they do not have any advanced training or are categorised as specialists if they have pursued an advanced training.

Type of facility: These are classified depending on the level of the care provided.

First level of care: Consists of clinics providing primary care on OPD basis by individual PPs (solo practice).

Second level of care: Nursing homes and hospitals 
We undertook a census of PPs and identified 316 formal allopathic PPs in Tumkur city. There was one NGO that was engaged in sputum collection and transportation. PPs prescribed medicines from private retail pharmacies. Retail private pharmacies dispensed medicines to patients on the presentation of a prescription by any doctor. However, over -the-counter sale, even for anti TB medicines, was quite common. PPs ordered diagnostic tests from private laboratories which varied in size from big chain of laboratories performing advanced diagnostic tests to standalone small laboratories, whose quality was suspect.

\subsection{Administration and Implementation of PPM Schemes}

The District TB officer is in charge of implementing the programme, including PPM activities, in the district. Under the PPM approach, the district TB officer is expected to line-list all the potential PPs in the district and foster coordination. The RNTCP field staff is expected to approach PPs to explain PPM schemes to the providers and explain the benefits that can accrue to them by collaborating with RNTCP. If the PP agrees to partner, then an outline of how the partnership would be operated will be discussed based on the PPM models. At the provincial level, the state TB officer provides oversight of the programme and allocates resources to the districts. The state TB officer is required to provide feedback to the Central TB Division, which is the policymaking body.

\section{STUDY OBJECTIVES}

\section{Main Objective}

To optimise the involvement of for-profit formal allopathic PPs in RNTCP in terms of referral of presumptive TB patients for sputum examination and notification of TB patients to RNTCP.

\section{Specific Objectives}

* To assess the involvement of PPs in RNTCP-PPM schemes.

* To document PP's TB management practices corroborated with patient's pathways to TB care, and chart PP's preferred modes of collaboration vis-à-vis the programme manager's expectations.

* To design, implement and assess the effectiveness of an intervention package to improve referrals of presumptive pulmonary TB patients from PPs to RNTCP for sputum smear microscopy diagnosis and improve the notification of TB patients by PPs to RNTCP. 


\section{STUDY DESIGN}

Both qualitative and quantitative research methods were used in three stages over a period of two years starting December 2012. In the first stage, we analysed the participation of PPs in RNTCP in 2011, through the review of RNTCP reports and lab registers maintained at the microscopic centres. In the second stage, we conducted in-depth interviews with stakeholders (PPs, TB patients and RNTCP officials) to understand the barriers for PPM implementation and the preferred modes of collaboration. Participant's recommendations were documented and analysed to design the intervention package. In the last phase starting September 2014, intervention was implemented between December 2014 and January 2016, by random allocation of for-profit allopathic PPs practising in the study area into two groups: one that received an intervention (receiving a package of interventions to improve referral and notification) and the other that did not receive any intervention (control arm). As main outcomes, we assessed the proportion of PPs referring presumptive pulmonary TB patients to RNTCP (referring PPs) and the proportion of PPs notifying TB patients on private treatment (notifying PPs) to RNTCP, in the intervention versus control group at the end of the intervention. As secondary outcomes, we measured the mean rate of referral of presumptive TB patients by PPs per year, and the crude and adjusted referral and pulmonary TB case-finding rate ratios were calculated with negative binomial regression.

\section{STUDY POPULATION}

The target population was for-profit formal allopathic PPs practising in Tumkur city. We categorised PPs into general practitioners without advanced training and specialists with advanced training. We further categorised specialists into (i) 'relevant': specialists who are potentially consulted by patients with chest symptoms such as chest physicians, general physicians (specialists in general medicine), surgeons, paediatricians, gynaecologists and (ii) 'non- relevant': specialists who are less likely to be consulted by patients with chest symptoms in their routine practice such as ENT surgeons, ophthalmologists, orthopaedics, dermatologists, radiologists and anaesthetists.

\subsection{Inclusion and Exclusion Criteria}

\section{Inclusion criteria}

* For-profit formal allopathic PPs practising permanently in Tumkur city.

\section{Exclusion criteria}

* PPs practising in Tumkur city less than once per week.

* PPs working in the government sector in the morning hours and running their private practice in the evening hours (not considered a priority, because they are assumed to be already exposed to RNTCP).

* 'Non-Relevant' specialists. 


\section{CONCEPTUAL APPROACH}

We relied on the Replicating Effective Programmes (REP) framework, to guide the design and implementation of our PPM model. While doing a literature review of the successful intervention packages to involve PPs in the public health programmes, the REP framework was identified to guide the implementation of the intervention package. The REP framework is essentially used for implementing evidence-based interventions into community-based settings through a combination of intervention 'packing', training, technical assistance and other strategies to maximise the chances for sustaining the interventions (Kilbourne et al. 2007). The framework has four phases: pre-conditions (identifying need, target population, and suitable intervention), pre-implementation (documentation and intervention packaging), implementation (package dissemination, training, technical assistance and evaluation), and maintenance and evolution (preparing the intervention for sustainability). We used the basic principles of the REP model to implement the intervention, and modified the activities based on our project needs. In the following paragraphs we will describe each phase in detail. See Figure 5 for the REP framework.

\section{Pre-conditions phase (2012-2013)}

In this first phase, PP's volume of referrals of pulmonary TB patients to RNTCP during 2011, was measured through the review of RNTCP reports and lab registers maintained at the microscopic centres. Systematic mapping of all the formal allopathic PPs in the district was carried out using

\begin{tabular}{|c|c|c|c|}
\hline $\begin{array}{l}\text { Pre-Conditions } \\
\text { - Identification of } \\
\text { the needs. } \\
\text { - Baseline } \\
\text { assessment of PP's } \\
\text { involvement in } \\
\text { RNTCP. } \\
\text { - Identification } \\
\text { of the effective } \\
\text { intervention } \\
\text { strategy. } \\
\text { - In-depth interviews } \\
\text { with PPs, TB } \\
\text { patients and } \\
\text { RNTCP staff, } \\
\text { and other key } \\
\text { stakeholders. }\end{array}$ & $\begin{array}{l}\text { Pre-Implementation } \\
\text { - Mapping of PPs, } \\
\text { DOTS providers } \\
\text { and microscopic } \\
\text { centres. } \\
\text { - Packaging the } \\
\text { intervention. } \\
\text { - Stakeholder } \\
\text { meetings to refine } \\
\text { the package. } \\
\text { - Development } \\
\text { of phone } \\
\text { directory, study } \\
\text { tools, training } \\
\text { and education } \\
\text { materials. }\end{array}$ & $\begin{array}{l}\text { Implementation } \\
\text { - Training of } \\
\text { RNTCP staff and } \\
\text { intervention PPs. } \\
\text { - Launch the } \\
\text { intervention: } \\
\text { Start data } \\
\text { collection, meeting } \\
\text { participants } \\
\text { periodically } \\
\text { improve the } \\
\text { communication } \\
\text { between PPs and } \\
\text { RNTCP. } \\
\text { - Ongoing support } \\
\text { and evaluation. }\end{array}$ & $\begin{array}{l}\text { Maintenance and } \\
\text { Evolution } \\
\text { - Analysis of the } \\
\text { impact of the } \\
\text { intervention. } \\
\text { - Workshop to } \\
\text { disseminate the } \\
\text { findings to PPs and } \\
\text { the RNTCP. } \\
\text { - Organisational } \\
\text { changes to } \\
\text { integrate } \\
\text { and sustain } \\
\text { intervention. }\end{array}$ \\
\hline
\end{tabular}

Figure 5: Replicating effective programme framework for intervention implementation 
different data sources and the PPs were stratified according to the following categories: (a) formal and informal; (b) system of medicine practiced (Allopathy or Indian system of medicine); (c) type of specialty; (d) type of health facility (solo, clinics and hospitals); and (e) urban and rural. As a second step, literature review was carried out to identify the existing evidence base, and effective interventions, which have produced statistically significant positive outcomes of interest (in this case, increased participation of PPs in the PPM activities).

\section{Pre-implementation (2013-2014)}

After assessing the participation of PPs in RNTCP, primary research was carried out using in-depth interviews with stakeholders (PPs, TB patients and RNTCP officials) to understand the barriers and preferred modes of collaboration. Participant's recommendations were analysed, and an intervention package was designed based on the empirical evidence gathered from the field. Before launching the intervention, the following activities were carried out to prepare the programme for implementing the project:

* Census of PPs practicing in Tumkur city was carried out using GPS technology from April to September 2014 by training three RNTCP TB-Health Visitors and one research staff. This enabled the programme to know the geographical location of PPs in the city. RNTCP staff visited PP's clinics and documented PP's characteristics using a checklist which was prepared by the PI, such as information on qualification, sex, age, years of experience, involvement with RNTCP as directly observed treatment provider, participation in RNTCP sensitisation workshop conducted in March 2014 in the district (prior and unrelated to our study), affiliation to a medical college and the average number of outpatient consultations/month were recorded. A total of 316 PPs were identified.

* To ensure that the intervention is adapted well in the field, a series of meetings were held with the district TB officer and sub-district RNTCP staff early in the study to establish shared values and alignment of purpose of the research study.

* Individual meetings were held with leading PPs (opinionmakers), who have influence on their peers, and the president of the Indian Medical Association, Tumkur chapter. We introduced the intervention package in such meetings to buy-in their ideas and received their inputs on the intervention package.

* To improve communication between PPs and RNTCP, study tools such as human resource directory with contact details of intervention PPs, key RNTCP staff, list of microscopic centres, and its in-charge medical officers, referral slips to facilitate referring of TB patients to RNTCP and notification forms were developed.

* Designing of TB-related education materials such as posters demonstrating symptoms of TB, diagnosis and standard treatment guidelines of TB, and the charts showing the availability of free TB services at government facilities were developed. These were meant to be displayed at PP's clinics. 


\section{Implementation (2015-2016)}

The intervention activities are broadly categorised into: activities targeted at strengthening RNTCP services and other set of intervention was targeted exclusively at PPs included in the intervention arm. The RNTCP staff implemented these activities under the supervision of the MO-TB control, Tumkur TU and District TB Officer. These activities are listed in the box below:

\section{Maintenance and Evolution (2016-2017)}

Our model used the existing manpower within the TB programme to carry out the research. The capacity of RNTCP staff was automatically built to involve PPs in RNTCP during the project, which enabled them to learn and adapt positive findings from the study. We identified resources for implementing strategies within RNTCP structure and reporting of PPM activities were integrated into the routine monitoring, which made the project self-sustainable. A workshop was held after the final analysis to communicate the findings to study participants and get their feedback. During this workshop, we highlighted the results on improved outcomes, strategies used for incorporating the activities into job duties, and training new personnel into the programme. This feedback was used to assist RNTCP staff to improve the services.

\section{Box 2: Summary of the Intervention Package}

\section{Activities targeted at General Strengthening of RNTCP Services}

* RNTCP staff refresher training on RNTCP guidelines and PPM schemes, sensitisation on the importance of collaboration and communication with PPs, detailed instructions on intervention components, procedures and tools;

* Strengthening of the whole information system, and in particular, documentation of the source of referrals in the district's laboratory registers maintained at sputum microscopic centre (training of laboratory technicians, supervision by senior TB laboratory supervisor);

* Streamlining the reporting of PPM activities with periodic monitoring of PP's referrals and staff meetings to discuss PPM activities;

* Improved accessibility of microscopic centres for patients, with extended opening hours, placement of signboards with directions to the microscopic centres in the health facilities, sensitisation of their helpdesk and registration counter staff to help patients reach microscopic centres;

* Counselling of patients by laboratory technicians of microscopic centres on going back to the treating physician with the sputum examination results and orientation about RNTCP treatment options and procedures;

* Provision of phone directory of study PPs to RNTCP staff and vice versa. 


\section{Activities Targeted Exclusively at PPs included in the Intervention Arm}

* Training: Half-day (2pm to $6 \mathrm{pm}$ ) training in standards of TB care, RNTCP guidelines, PPM schemes, how to become a DOT provider, notification of TB patients, intervention components, procedures and tools; PPs were invited by the DTO, the meeting was held at Indian Medical Association in Tumkur city; Karnataka Medical Association Continuing Medical Education credit points from Karnataka Medical Council were awarded to participating PPs; the training was led by RNTCP staff.

* Systematic visits by DTO to clinics of intervention PPs who did not participate in the training to secure participation, communicate essential content of what was covered during the halfday training, and provide study tools.

* Strengthening communication between RNTCP and PPs through:

- $\quad$ Sending SMSs with results of sputum examination and information on whether the referred patients have started RNTCP treatment;

- Monthly visits to the PPs' clinics by TB-Health Visitors, to provide feedback on referred patients and retrieve and replenish referral slips and notification forms;

- $\quad$ Provision of phone directory of key RNTCP staff;

- $\quad$ Provision of TB information-education-communication material for patients and maps with directions of microscopic centres.

\section{DATA COLLECTION AND ANALYSIS}

\subsection{DATA COLLECTION}

\subsubsection{Pre-Condition phase}

Quantitative data was collected in 2012 to assess PP's participation in RNTCP in 2011. Data on referrals were collected retrospectively from the microscopic centres' laboratory registers. Individual names of referring PPs, number of referrals, total number of patients undergoing sputum examination (referred and non-referred by PPs) and numbers with a positive smear result were extracted and entered into an Excel database (Micro- Soft, Redmond, WA, USA). Data was cross verified with routine quarterly reports and records at the DTC. Information on whether and how many PPs had engaged in PPM schemes in the district was looked up in the routine DTC reports and registers. This was followed by qualitative data collection in the next phase.

\subsubsection{Pre-implementation phase}

In-depth interviews were conducted with a variety of stakeholders such as TB patients, PPs, RNTCP staff, and the programme managers, sequentially. Data was collected from May 2013 to August 2013. Tools were pilot tested in February 2013 and fine-tuned accordingly. In order to elicit quality data, interviews were preceded by warm-up visits to interviewees by a field coordinator, who shared 
the information brochure and explained about the research project in the local language, Kannada. Written consent was taken from the participants. Efforts were made to build rapport with patients and an appointment was sought for interviews. Participants were given the choice to choose a date, time and place convenient for them. The PI conducted in-depth interviews in local language Kannada, which lasted for 45 minutes to one-and-a-half hours.

\section{(A) Interviews with patients}

A total of 33 in-depth interviews were conducted on a purposive sample of new TB patients. Patients were recruited from three different groups, with an objective to document patient's navigation in the health system to reach RNTCP services and their experiences of completing RNTCP treatment: (1) patients who reached RNTCP directly on their own and took DOTS at RNTCP; (2) patients who were referred by PPs to RNTCP and took DOTS at RNTCP; and (3) patients who were diagnosed by RNTCP and took DOTS from PPs, irrespective of how they initially reached RNTCP. Patients were further stratified for rural $(n=18)$ and urban (15) settings. Patients were shortlisted from RNTCP laboratory registers. Patient characteristics such as whether they reached RNTCP directly or were referred by a PP or taking DOTS from a PP, duration of the treatment, etc., were cross-checked with RNTCP TB registers and treatment cards. To reduce the recall bias, we selected patients who had completed their treatment in the last three months or who were about to complete the treatment in the next one month at the time of the interview. Three interview guides, one for each category of patients, were developed.

\section{(B) Interviews with PPs}

After assessing the actual number of PPs referring presumptive TB patients to microscopic centres, a total of 38 PPs were selected purposively from the list $(n=198)$ maintained at the district TB centre. PPs were then categorised into three groups based on the referrals into: (i) actively-referring PPs: who had referred 12 or more than 12 patients $(\mathrm{n}=17)$, (ii) minimally-referring PPs: who had referred at least one, but less than 12 patients $(n=8)$ and (iii) not-referring PPs: who had not referred any case $(\mathrm{n}=13)$. Though, we considered all 19 actively-referring PPs, we could interview 17 of them, since two of them were out of town during the study period. Efforts were made to match nonreferring and minimally-referring PPs with actively-referring PPs for age, qualification, patient load and location of practice. Three interview guides; one for each category of was used.

\section{(C) Interviews with RNTCP managers}

In-depth interviews were conducted with programme managers $(n=2)$, administrators at the national level $(n=2)$, and technocrats who provide technical assistance to RNTCP $(n=4)$. Tools for these interviews were developed at a later stage after consolidating the issues and themes emerging from the earlier interviews.

Two FGDs were conducted with RNTCP field staff; one each for senior treatment supervisors and TB-health visitors $(n=10)$ and the other with senior TB laboratory supervisors $(n=6)$ to elicit the challenges they face while involving PPs in RNTCP. The FGDs were conducted before PP's 
interviews, which helped refining the PP's interview tools. The duration of FGDs was about oneand-a-half hours each.

\subsubsection{Intervention phase}

Data on referrals and notifications from December 2014 to January 2016 (14 months) were collected. In the paragraph below, we explain the study instruments used for data collection by RNTCP staff:

* The senior TB laboratory supervisor visited the microscopic centres every fortnight to supervise whether PP's referrals were recorded by the laboratory technicians in the laboratory registers maintained at the microscopic centres. At the end of every month, the senior TB laboratory supervisor compiled PP's contribution to RNTCP in terms of presumptive pulmonary TB patients referred by PPs using a reporting format and shared this information with the district TB office and the staff implementing PPM.

* The senior treatment supervisor supervised TB-HVs using the checklist. At the end of every month, the senior treatment supervisor compiled PPs contribution to RNTCP in terms of PP's referred patients started on RNTCP treatment and the place of DOT provision using a checklist. This information was shared with the district TB office and the staff implementing PPM on a monthly basis.

* TB- health visitors visited PP's health facilities in their respective areas to cover four zones (South, North, West and East), using the checklist in Annexure 8. They collected carbon copies of referral slips and the notification forms. At the end of every month these were handed over to the data entry operator.

* MO-TB control ensured the data collection from RNTCP staff by using the checklist.

All the data collected by the staff were entered in a Microsoft Excel (2010) sheet monthly by a trained data entry operator.

\subsection{DATA ANALYSIS}

\subsubsection{Quantitative data analysis}

Quantitative data collected during the pre-condition phase were analysed using MS Excel v. 2010 (Microsoft) and Epi Info v. 3.5.3 (Centers for Disease Control and Prevention, Atlanta, GA, USA). The median number of referrals per PP and the interquartile ranges were calculated considering only PPs who had referred at least one case during the study period.

Quantitative data from the intervention phase regarding the effectiveness of involving PPs with RNTCP was measured at the beginning and at the end of the intervention. Quantitative data was entered systematically in excel. Comparisons were made between the intervention and control groups. The difference in the proportion of referring PPs and the median number of referrals per 
PP per year between the study arms, with 95\% confidence interval (95\% CI) (bootstrapped for medians), were calculated. Crude referral and PTB case-finding rates and crude and adjusted rate ratios with $95 \%$ CI were calculated with negative binomial regression. Analyses were performed using IBM SPSS Statistics Version 23.0.

\subsubsection{Qualitative data analysis}

All interviews were audio recorded and transcribed verbatim by professional transcribers. The PI crosschecked each transcript, looked for missing content, and field notes were added wherever applicable. A combination of deductive and inductive approaches was used to analyse data. The deductive approach was based on the research questions, which primarily aimed to examine the factors influencing TB patient's therapeutic itineraries to RNTCP and understand the cross-referral practices of PPs to RNTCP. However, we kept the flexibility of including new themes emerging from the data (inductive approach). This was supplemented by an inductive 'grounded theory' approach seeking to elicit respondent-generated meanings of collaboration (Strauss A 1997). An initial coding scheme was tested on a handful of interviews, which were then refined and organised at a broader conceptual level into themes by grouping them together (Herbert J. Rubin \& Irene S. Rubin 2005) (Burnard, et al. 2008). We systematically checked across the transcripts for the consistency of these emerging categories. We conducted a thematic analysis (MQ 2002), (Boyatzis 1998), (MQ 2002) to develop a logic model depicting the contextual barriers to engage PPs in RNTCP. In later stages of the analysis, we explored the relationships between the themes and across different categories of participants to identify patterns in the data. The analysis was conducted with the support of QSR Vivo 9 (QSR International Pvt LTD, Melbourne, Australia). Memos were written on patient's navigation to RNTCP, PP's cross-referral practices of patients to RNTCP and RNTCP staff barriers for collaboration, and the data were corroborated. We then explored the relationships between and across the themes across different categories of participants. Major recurring themes were grouped, and memos were developed based on the data of each category and sub-categories of respondents. We identified such quotes that best supported each domain. Finally we examined the patterns and linkages between themes and respondents' attributes to explore the challenges for collaboration between and across different study participants (Eisenhardt and Graebner 2007). Major recurring themes were grouped, and memos were developed based on the data of each category, and sub-categories of respondents.

\section{ETHICAL APPROVAL}

The study received ethical approval from the institutional ethics committee, Institute of Public Health, Bangalore, and institutional review board of Institute of Tropical Medicine, Antwerp, Belgium.

\section{TRIAL REGISTRATION}

The study is registered in the Clinical Trial Registry, India (CTRI) under the registration number CTRI/2017/09/ 009672. 


\section{PART III - ORIGINAL RESEARCH}

This part of the thesis brings findings from three sub-studies representing chronologically the linked phases of doctoral research. The findings are subdivided into four chapters. Each chapter uses a manuscript published in peer-reviewed journals. 


\section{CHAPTER 1}

This chapter sheds light on the level of PP's participation in the PPM strategy and allow us to know the pre-conditions to design an intervention to optimise the involvement of PPs in RNTCP. The paper titled, 'Private practitioners' contributions to the Revised National Tuberculosis Control Programme in a South Indian district', aimed to assess the participation of for-profit, formal PPs under RNTCP's PPM schemes and document their contribution to RNTCP pulmonary TB case finding.. 


\title{
Private practitioners' contributions to the Revised National Tuberculosis Control Programme in a South Indian district
}

\author{
V. Yellappa, ${ }^{\star \star}$ T. Battaglioli, ${ }^{\dagger}$ S. K. Gurum, ${ }^{\star}$ D. Narayanan, ${ }^{*}$ P. Van der Stuyft ${ }^{\dagger \S}$ \\ *Institute of Public Health, Bangalore, India; 'Department of Public Health, Institute of Tropical Medicine, \\ Antwerp, Belgium; ; ${ }^{7}$ Department of Health and Family Welfare, Bangalore, India; ${ }^{5}$ Department of Public Health, \\ Faculty of Medicine, Ghent University, Ghent, Belgium
}

SETTING: Tumkur District, South India.

OBJECTIVE: To assess the participation of for-profit, formal private practitioners (PPs) under the Revised National Tuberculosis Control Programme's (RNTCP's) public-private mix (PPM) schemes and document their contribution to RNTCP pulmonary tuberculosis (TB) case finding.

DESIGN : RNTCP reports at district TB centre were reviewed. PPs were mapped and their referrals of presumptive TB cases to the RNTCP during 2011 were assessed using laboratory registers at designated microscopy centres (DMCs).

RESULTS: None of the 424 PPs had signed up for any PPM scheme. However, $22 \%$ made at least one referral to a DMC in 2011. PP referrals constituted
$15 \%$ of the presumptive TB cases examined at the DMCs, and PPs contributed to $23 \%$ of the sputum smear-positive TB cases detected. Among PP referrals, the proportion of confirmed smear-positive cases was high $(24 \%)$.

CONCLUSION: Fifteen years after the start of PPM, formal engagement of PPs with RNTCP was nonexistent. However, PPs do refer cases to the RNTCP and contribute to a fraction of TB case detection. The high proportion of confirmed sputum smear-positive cases suggests that PPs tend to make selective referrals. More efforts are needed to promote the engagement of PPs in the RNTCP.

KEY WORDS: private practitioners; public-private mix schemes; pulmonary tuberculosis; referrals; RNTCP
TUBERCULOSIS (TB) remains a major public health problem in India, with an estimated annual incidence of 171 cases per 100000 population in 2013, accounting for $24 \%$ of all TB cases worldwide. ${ }^{1}$ The Indian Revised National TB Control Programme (RNTCP), based on the World Health Organization (WHO) recommended DOTS strategy, was launched in 1996. The country's health system is complex, with diverse providers ranging from unqualified practitioners to highly trained specialists. The private health sector is dominant and largely unregulated.

There is a large body of evidence to demonstrate that private sector providers (PSPs) are often the first choice for seeking TB care in India, ${ }^{2-5}$ with $50 \%$ of cases being managed in the private sector, ${ }^{6}$ where TB is often inaccurately diagnosed and ineffectively treated. ${ }^{7-11}$ Recognising the critical need to engage PSPs in RNTCP, the Government of India published guidelines for non-government organisations' (NGOs') and allopathic private practitioners' (PPs') involvement in TB control in 2000 and 2001 respectively, which were revised in 2008. There are currently 10 public-private mix (PPM) schemes, principally based on results-based financing (Table 1). Each scheme has specific objectives that the partnership is expected to fulfil by signing a memorandum of understanding with the District TB Officer (DTO). ${ }^{12}$ The Government of India declared TB a notifiable disease in May 2012, making it mandatory for all public and private providers to notify TB cases to the designated public health authorities. Nevertheless, notification from PPs remains abysmal. ${ }^{13}$

Studies of PPM initiatives in India have demonstrated their positive impact on case detection and treatment success rate and their feasibility and costeffectiveness, ${ }^{14-17}$ but these are confined to a specific setting or context. Lessons from these successful experiences have not been pursued or scaled up across the country. Formal engagement of PPs in the RNTCP through any available PPM scheme is low: in 2010 only 10000 PPs in the entire country were officially engaged. ${ }^{18,19}$ Moreover, no studies have documented the participation and contribution of individual PPs to the RNTCP under programme conditions.

We conducted a study to assess whether for-profit,

Correspondence to: Vijayashree Yellappa, Institute of Public Health, No 250 Master's Cottage, $2^{\text {nd }} \mathrm{C}$ Main, $2^{\text {nd }}$ 'C' Cross, Girinagar $1^{\text {st }}$ Phase, Bangalore 560085, Karnataka, India. e-mail: vijayashree@iphindia.org

Article submitted 1 September 2015. Final version accepted 27 November 2015. 
Table 1 PPM schemes and related financial incentives in the RNTCP

1 Advocacy, communication and social mobilisation: for NGOs to mobilise local political commitment and resources for TB, empower communities affected by TB grant-in-aid: USD2293 per one million population per year

2 Sputum collection centre: for sputum sample collection in 'underserved' areas grant-in-aid: USD917 per year, per centre

3 Sputum pick up and transport service: for NGOs to transport sputum samples to the nearest DMCs grant-in-aid: USD367 per year

4 DMC scheme: for $\mathrm{NGO}$ /private laboratories to provide sputum smear microscopy and anti-tuberculosis treatment services free of charge

grant-in-aid: USD2292 per year; only microscopy, USD0.4 per slide

5 Laboratory technician scheme: to recruit laboratory technicians to strengthen RNTCP diagnostic services in hospitals outside ministry of health grant-in-aid: Laboratory technician's salary and 5\% overhead cost

6 Culture and DST: for well-functioning mycobacterial culture and DST laboratory in the private/NGO sector grant-in-aid: USD31 per specimen

7 Adherence scheme: for NGOs and PPs to ensure patient adherence to anti-tuberculosis treatment

grant-in-aid for NGOs: USD612 for 100000 population per year; for PPS: USD6 per patient successfully treated

8 Slum scheme: for NGOs/self-help groups/PPs working in slums to ensure timely diagnosis and treatment adherence grant-in-aid: USD765 per 20000 population per year

9 Tuberculosis Unit model: for NGOs who can carry out all the services typically executed by RNTCP TB Units grant-in-aid: USD8103 per year

10 TB-HN scheme: for NGOs already working with HIV patients to help treat TB in conjunction with HIV treatment grant-in-aid: USD1834 per NGO per 1000 target population

PPM = public-private mix; RNTCP $=$ Revised National Tuberculosis Control Programme; $N G O=$ non-governmental organisation; $T B=$ tuberculosis; USD $=$ US dollar; DMC = designated microscopy centre; DST = drug susceptibility testing; PP = private practitioner; $\mathrm{HIV}=$ human immunodeficiency virus.

formally trained PPs working in solo clinics, nursing homes and hospitals in a district in South India are engaged in RNTCP PPM schemes and to document whether and to what extent, either formally or informally, they contribute to the RNTCP in terms of new/retreatment smear-positive pulmonary TB case finding.

\section{MATERIALS AND METHODS}

\section{Setting}

Tumkur District (total population 2716997) is located in Karnataka, South India. As elsewhere in India, the district has a public sector network of primary health centres (PHCs) and hospitals that provide free health care. As of 2012, there were 146 PHCs and 10 hospitals providing a mix of primary and secondary care. There is also a large private health sector, with a wide array of health care providers ranging from unqualified practitioners to highly trained specialists.

Under the umbrella of the RNTCP, the district is divided into six TB Units (TUs), each catering for a population of around 500000 and responsible for programme implementation. Under each TU, designated microscopy centres (DMCs) cover a population of 100000 and perform sputum smear microscopy ( $n$ $=28$ ). Patients can either access these centres directly or be referred by a public facility or a PP. DMC laboratory technicians are expected to record various details, including the source of referral, for each presumptive TB case examined. Quarterly reports are generated at the District TB Centre (DTC), based on the reports from TUs.

By 2011, the DTO had carried out four workshops at the district's headquarters on sensitisation about the RNTCP for allopathic PPs, as reported in the records at the DTO office.

\section{Definitions}

For our study, PPs were defined as 'formal' if they were formally trained in allopathic medicine or in the Indian system of medicine-Ayurveda, Yoga and Naturopathy, Unani, Sidda and Homeopathy (AYUSH), and as 'informal' otherwise. Allopathic PPs with no specialist training were classified as general practitioners (GPs). Specialists potentially consulted by patients with chest symptoms in their routine practice, such as physicians, surgeons, paediatricians and gynaecologists, were categorised as 'Core'; other specialists less likely to be consulted, such as anaesthetists, psychiatrists and orthopaedicians, etc., were categorised as 'Others'.

\section{Data collection and analysis}

Data were collected in 2012 to assess PP participation in the RNTCP in 2011. Data on referrals were collected retrospectively from the DMC laboratory registers. Individual names of referring PPs, number of referrals, total number of patients undergoing sputum examination (referred and non-referred by PPs) and numbers with a positive smear result were extracted and entered into an Excel database (MicroSoft, Redmond, WA, USA). Data were cross-verified with routine quarterly reports and records at the DTC. Information on whether and how many PPs had engaged in PPM schemes in the district was searched for in routine DTC reports and registers.

To ascertain the total number of PPs practising in the study area, regardless of their engagement with the RNTCP, PPs were mapped using five data sources: the Indian Medical Association (New Delhi, India), the Karnataka Private Medical Establishment Act, the district TB office, private nursing homes and the Medical College, complemented by field visits. Of the 28 DMCs, only five (one urban, four rural), all located in the headquarters of the respective TUs, systematically recorded the source of referrals in their laboratory registers: Sira Town DMC, Koratagere Town DMC, Kunigal Town DMC, Swami Vivekananda Integrated Rural Health Centre DMC in Pavagada Town and DTC DMC in Tumkur City. 
Table 2 Number of PPs practising in the five selected TB Units found in each of five different data sources, Tumkur District, Karnataka, India, 2011

\begin{tabular}{|c|c|c|c|c|c|c|}
\hline \multirow[b]{2}{*}{ TB Unit } & \multirow{2}{*}{$\begin{array}{c}\text { Mapped PPs } \\
n\end{array}$} & \multicolumn{5}{|c|}{ PPs found in each data source, $n(\%)^{*}$} \\
\hline & & KPMEA & IMA & DTC & Nursing home list & Medical college \\
\hline Koratagere & 20 & $6(30)$ & $4(20)$ & $15(75)$ & 0 & 0 \\
\hline Kunigal & 27 & $19(70)$ & $11(41)$ & $8(30)$ & $1(4)$ & 0 \\
\hline Pavagada & 23 & $12(52)$ & $13(57)$ & $15(65)$ & $9(39)$ & 0 \\
\hline Sira & 45 & $23(51)$ & $16(36)$ & $18(40)$ & $1(2)$ & 0 \\
\hline Tumkur & 309 & $157(51)$ & $160(52)$ & $115(37)$ & $58(19)$ & $73(24)$ \\
\hline All & 424 & $217(51)$ & $204(48)$ & $171(40)$ & $69(16)$ & $73(17)$ \\
\hline
\end{tabular}

* Some PPs were found in more than one data source.

$\mathrm{PP}=$ private practitioners; $\mathrm{TB}=$ tuberculosis; $\mathrm{KPMEA}=$ Karnataka Private Medical Establishment $\mathrm{Act} ; \mathrm{IMA}=$ Indian Medical Association; DTC $=$ District TB Centre

The analysis was thus narrowed down to these five DMCs, and the mapping of PPs was restricted to the five TUs where they were located. Of the remaining 23 DMCs, 2 were urban and 21 rural.

Data were analysed using MS Excel ${ }^{\circledR}$ v. 2010 (Microsoft) and Epi Info ${ }^{\text {TI }}$ v. 3.5 .3 (Centers for Disease Control and Prevention, Atlanta, GA, USA). The median number of referrals per PP and the interquartile ranges (IQR) were calculated considering only PPs who had referred at least one case during the study period. Two parallel analyses were performed, including and excluding 44 PPs not found during the field visit. If not expressly stated, the reported results include the 44 PPs.

As the study involved the collection of secondary data from routine RNTCP registers and reports, and personally identifiable information of patients was not collected, ethical approval was not required.

\section{RESULTS}

A total of 424 formal PPs (363 allopaths and 61 AYUSH) were identified (Table 2). None of the five mapping sources was complete, and there were some overlaps. Of the 424 PPs mentioned in one or more data sources, 44 were not found during the field visit, possibly because they had relocated or retired; six of these had made at least one referral in 2011. Among 375 PPs with complete information on the site of practice, the majority of the GPs ( $n=117,84 \%$ ) practised in solo clinics; among specialists, 134 $(57 \%)$ practised in a nursing home or hospital. No PP in the entire district of Tumkur had formally signed up for any of the PPM schemes during 2011.

Among the 424 PPs mapped in the study area, 94 $(22 \%)$ had made at least one referral to a DMC during the year (Table 3): 87 allopaths (32 [38\%] GPs and $55[20 \%]$ specialists $-25 \%$ core and $7 \%$ other specialists) and $7(11 \%)$ AYUSH. Results did not significantly change on excluding the 44 PPs not found during the field visit. A total of 675 presumptive TB cases were referred by PPs (Table 4) to the five DMCs, $320(47 \%)$ by GPs, $336(50 \%)$ by specialists ( $97 \%$ of whom were core specialists) and $19(3 \%)$ by
AYUSH. Among the referring PPs, the median number of referrals per PP was 3 (IQR 1-6) (Table 4).

Among the total 4446 presumptive TB cases examined at the five DMCs, $675(15 \%)$ had been referred by PPs (Table 5 ). There were respectively 157 $(23 \%)$ and $521(14 \%)$ confirmed smear-positive cases among those referred and not referred by PPs, and respectively $81(24 \%)$ and $74(23 \%)$ among referrals by core specialists. In the 23 DMCs not included in the study, $1246(7 \%)$ of the 17540 presumptive TB cases examined were confirmed as smear-positive cases. PPs thus contributed to $23 \%$ of all detected sputum smear-positive TB cases in the study area, with variations across the different DMCs (Table 5).

\section{DISCUSSION}

Even 15 years after the introduction of PPM schemes, we found a complete absence of formal engagement by PPs with the RNTCP throughout Tumkur District. However, in the study area, $22 \%$ of PPs had made at least one referral to the RNTCP, contributing nearly a quarter of the sputum smear-positive TB cases detected. Compared to core specialists and GPs, a lower number of other specialists and AYUSH referred cases to the RNTCP. The proportion of confirmed sputum smear-positive cases among presumptive TB cases referred by PPs was much higher than the normally expected value of $10 \% .^{20}$

This is the first study in India to assess the participation and contribution of for-profit, formally trained PPs in the RNTCP under a programme setting. An important limitation of the study is that it included only five DMCs that systematically documented sources of referral. Some patients referred by PPs might have presented to other DMCs, possibly leading to an underestimation of the contribution of PPs to referrals. On the other hand, this is likely to be low, as the selected DMCs are located at the headquarters of their respective TUs, where the majority of the district's PPs have their practice. Another limitation is that as data were extracted from DMC laboratory registers, referred cases who did not reach the DMCs were not captured, hence potentially leading to an underesti- 
Table 3 Total number of mapped PPs and number and proportion referring presumptive tuberculosis cases for spulum smear microscopy examination to five selected DMCS, Tumkiur District, Kamataka, india, 2011

\begin{tabular}{|c|c|c|c|c|c|c|c|c|c|c|c|c|c|c|c|}
\hline \multirow[b]{4}{*}{ DMAC } & \multirow[b]{4}{*}{$\begin{array}{l}\text { Population } \\
2011\end{array}$} & & & \multicolumn{10}{|c|}{ Alopaths } & \multirow{2}{*}{\multicolumn{2}{|c|}{ ArusH }} \\
\hline & & \multicolumn{2}{|c|}{ Al PAs } & \multicolumn{2}{|c|}{ All } & \multicolumn{2}{|c|}{$G P$} & \multicolumn{6}{|c|}{ Specialints } & & \\
\hline & & & & & & & & & All & & one & & hes & \multirow[b]{2}{*}{$\begin{array}{c}\text { Iotal } \\
n\end{array}$} & \multirow[b]{2}{*}{$\begin{array}{l}\text { Referening } \\
n(5 \%)\end{array}$} \\
\hline & & $\begin{array}{c}\text { lotal } \\
n\end{array}$ & $\begin{array}{c}\text { Refering } \\
n(\%)\end{array}$ & $\begin{array}{c}\text { Total } \\
n\end{array}$ & $\begin{array}{c}\text { Rederering } \\
n(\%)\end{array}$ & $\begin{array}{c}\text { Iotal } \\
n\end{array}$ & $\begin{array}{l}\text { Referring } \\
n(\%)\end{array}$ & $\begin{array}{c}\text { Total } \\
n\end{array}$ & $\begin{array}{c}\text { Reterring } \\
n(\%)\end{array}$ & $\begin{array}{c}\text { Iotal } \\
n\end{array}$ & $\begin{array}{c}\text { Referring } \\
n(\%)\end{array}$ & $\begin{array}{c}\text { Total } \\
n\end{array}$ & $\begin{array}{c}\text { Reterring } \\
n(\%)\end{array}$ & & \\
\hline Koralogere fown & 17021 & 20 & $5(25)$ & 12 & $3(25)$ & 7 & $2(29)$ & 5 & $1(20)$ & 4 & $1(25)$ & 1 & 0 & 8 & $2(25)$ \\
\hline Kunigal fown & 39721 & 27 & $6(22)$ & 23 & $6(26)$ & 10 & $3(30)$ & 13 & $3(23)$ & 10 & $3(30)$ & 3 & 0 & 4 & 0 \\
\hline Pavagada SVRHCC & 67235 & 23 & $4(1 n)$ & $n$ & $4(18)$ & 9 & $3(33)$ & 13 & 1 (8) & 8 & 1 (13) & 5 & 0 & 1 & 0 \\
\hline Sira fown & 66520 & 45 & $20(44)$ & 34 & $20(59)$ & 15 & $11(73)$ & 19 & $9(47)$ & 16 & $8(50)$ & 3 & 1(33) & 11 & 0 \\
\hline Tumkur DTC & 305821 & 309 & 59 (19) & 272 & $54(20)$ & 43 & $13(300)$ & 229 & $41(18)$ & 155 & $36(23)$ & 74 & $5(7)$ & 37 & 5 (14) \\
\hline Total & 496318 & 424 & $94(22)$ & 353 & $87(24)$ & 84 & $32(33)$ & 279 & $55(20)$ & 193 & $49(25)$ & 85 & $6(7)$ & 61 & $7(11)$ \\
\hline
\end{tabular}

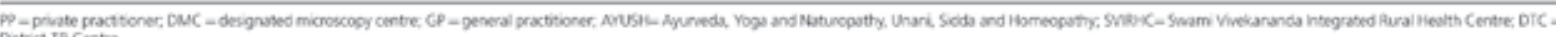
Destret IB Cente

Table 4 Volume of referrals by PAs to the selected DMCS for sputum smear microscopy examination, Tumkur Distrikt, Kamataka, hdia, 2011

\begin{tabular}{|c|c|c|c|c|c|c|c|c|c|c|c|c|c|c|}
\hline \multirow[b]{4}{*}{ DMC } & \multirow{3}{*}{\multicolumn{2}{|c|}{ Pp referrats }} & \multicolumn{10}{|c|}{ Referrats by allopoths } & & \\
\hline & & & \multirow{2}{*}{\multicolumn{2}{|c|}{ Al }} & \multirow{2}{*}{\multicolumn{2}{|c|}{ GPS }} & \multicolumn{6}{|c|}{ Specialsts } & \multirow{2}{*}{\multicolumn{2}{|c|}{ Beferras by AYUSH }} \\
\hline & & & & & & & \multicolumn{2}{|r|}{ Al } & \multicolumn{2}{|r|}{ Coce } & \multicolumn{2}{|r|}{ Others } & & \\
\hline & $\begin{array}{c}\text { Total } \\
n\end{array}$ & $\begin{array}{l}\text { Number per pp } \\
\text { median [1. }[\mathrm{CN}]\end{array}$ & $\begin{array}{c}\text { Total } \\
n\end{array}$ & $\begin{array}{l}\text { Number per PP } \\
\text { median }[\mid \not C A]\end{array}$ & $\begin{array}{c}\text { botal } \\
n\end{array}$ & $\begin{array}{l}\text { Number per } P p \\
\text { median }[10 \%]\end{array}$ & $\begin{array}{c}\text { Total } \\
n\end{array}$ & $\begin{array}{l}\text { Number per } P p \\
\text { median }[D Q A]]\end{array}$ & $\begin{array}{c}\text { Votal } \\
n\end{array}$ & $\begin{array}{c}\text { Number per Pp } \\
\text { median [DQA] }\end{array}$ & $\begin{array}{c}\text { Total } \\
n\end{array}$ & $\begin{array}{c}\text { Number per } P \text { P } \\
\text { median [1QP] }\end{array}$ & $\begin{array}{c}\text { Total } \\
n\end{array}$ & $\begin{array}{c}\text { Number per PP } \\
\text { median }[1 Q R]\end{array}$ \\
\hline $\begin{array}{l}\text { Koratagere Town } \\
\text { Kunigal Town } \\
\text { Pavagada SViavic } \\
\text { Sra fown } \\
\text { Tumkur otC }\end{array}$ & $\begin{array}{r}33 \\
104 \\
21 \\
202 \\
315\end{array}$ & $\begin{array}{l}2011.0-140 \mid \\
7012.0-140 \mid \\
55[2.5-8.0] \\
50 \mid 1.5-120] \\
30[1.0-5.0]\end{array}$ & $\begin{array}{r}30 \\
104 \\
21 \\
202 \\
299\end{array}$ & $\begin{array}{l}140[1.0-15.0] \\
70120-140 \mid \\
5.5[25-8.01 \\
5.011 .512] \\
3.0[1.0-5.01\end{array}$ & $\begin{array}{r}15 \\
76 \\
14 \\
162 \\
53\end{array}$ & $\begin{array}{l}7.5[1.0-140] \\
20[1.0-730] \\
4.0[1.0-9.0] \\
9.0[5.0-30.0] \\
2.0[1.0-4.0]\end{array}$ & $\begin{array}{r}15 \\
28 \\
7 \\
40 \\
246\end{array}$ & $\begin{array}{c}15 \\
11.0[3.0-14.0] \\
7 \\
1.0[1.0-5.0] \\
3.0[1.0-5.0]\end{array}$ & $\begin{array}{r}15 \\
28 \\
7 \\
35 \\
240\end{array}$ & $\begin{array}{c}15 \\
11.0[3.0-14.0] \\
7 \\
1.0[1.0-6.5] \\
3.0[2.0-6.0]\end{array}$ & $\begin{array}{l}0 \\
0 \\
0 \\
5 \\
6\end{array}$ & $\begin{array}{c}0 \\
0 \\
0 \\
5[-1 \\
1.0[1.0-1.0]\end{array}$ & $\begin{array}{r}3 \\
0 \\
0 \\
0 \\
16\end{array}$ & $\begin{array}{c}1.5[1.0-2.0 \mid \\
0 \\
0 \\
0 \\
3.0[2.0-6.0]\end{array}$ \\
\hline lotal & 675 & $30[1.0-6.0]$ & 656 & $3.0[1.0-7.0]$ & 320 & 4 [2-10.5] & 336 & $3.0[1.0-6.0]$ & 325 & $3.0[2.0-6.0]$ & 11 & $1.0[1.0-2.0]$ & 19 & $2.0[1.0-4.0]$ \\
\hline
\end{tabular}

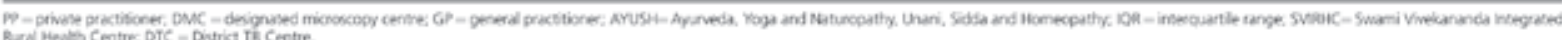


Table 5 Number of presumptive TB cases examined by sputum smear microscopy, source of referral and number found smearpositive in five selected DMCs in Tumkur District, Karnataka, India, 2011

\begin{tabular}{|c|c|c|c|c|c|}
\hline \multirow[b]{2}{*}{ DMC } & \multicolumn{2}{|c|}{ Presumptive TB cases examined } & \multicolumn{3}{|c|}{ Confirmed sputum smear-positive TB cases } \\
\hline & $\begin{array}{c}\text { Total } \\
n\end{array}$ & $\begin{array}{c}\text { PP referrals } \\
n(\%)\end{array}$ & $\begin{array}{c}\text { Total smear-positive } \\
n(\%)\end{array}$ & $\begin{array}{c}\text { Confirmed smear-positive } \\
\text { PP referrals } \\
n(\%)\end{array}$ & $\begin{array}{c}\% \text { of total confirmed } \\
\text { smear-positive cases } \\
\text { referred by PPs }\end{array}$ \\
\hline Koratagere Town & 511 & $33(6)$ & $55(11)$ & $5(15)$ & 9 \\
\hline Kunigal Town & 379 & $104(27)$ & $50(13)$ & $16(15)$ & 32 \\
\hline Pavagada SVIRHC & 1244 & $21(2)$ & $215(17)$ & $12(57)$ & 6 \\
\hline Sira Town & 1174 & $202(17)$ & $184(16)$ & $56(28)$ & 30 \\
\hline Tumkur DTC & 1138 & $315(28)$ & $174(15)$ & $68(22)$ & 39 \\
\hline Total & 4446 & $675(15)$ & $678(15)$ & $157(23)$ & 23 \\
\hline
\end{tabular}

$\mathrm{TB}=$ tuberculosis; $\mathrm{DMC}=$ designated microscopy centre; $\mathrm{PP}=$ private practitioner; $\mathrm{SVIRHC}=$ Swami Vivekananda Integrated Rural Health Centre; $\mathrm{DTC}=\mathrm{District} \mathrm{TB}$ Centre.

mation of the volume of PP referrals. Laboratory technicians at the selected DMC might also have missed documenting the source of referral in some cases, also leading to an underestimation of PP referrals. Underreporting of the source of referral at the DMC level could have been estimated by interviewing a sample cohort of PPs on their referral patterns; however, this was not done. Another limitation was that we focused on PP referral for sputum smear microscopy for the diagnosis of presumptive pulmonary TB without exploring referral patterns for the diagnosis of presumptive extrapulmonary TB. A recent study in the same area showed that nearly half of TB cases diagnosed and/or treated in private clinics/hospitals are extra-pulmonary. ${ }^{21}$ It should be noted, however, that at the time of the study histopathology was available only in the private sector in the district, making referral to the RNTCP for the diagnosis of extra-pulmonary TB not meaningful to explore.

The WHO recommends that the source of referral and place of treatment be routinely recorded and reported to assess PSP participation in national TB programmes. ${ }^{22}$ This helps to estimate the contribution of PPs to the RNTCP and prioritise efforts to engage PPs who are most likely to contribute to RNTCP. Although mandated to do so by the programme, laboratory technicians at peripheral DMCs did not routinely comply with this recommendation; only those at the DMCs included in the study complied. Furthermore, the format of the laboratory register is not adapted to this guideline, suggesting that this practice needs to be strengthened by the RNTCP.

A fully comprehensive registry of PPs for the district was not available. The list of PPs available in the DTO contained only $40 \%$ of the PPs that we ultimately mapped. The majority of GPs practised in solo clinics, which were more challenging to identify and locate. Systematic mapping of all health care providers is a prerequisite for an effective PPM strategy, ${ }^{23}$ particularly in India's pluralistic private health sector. Clear guidelines should be developed by the Government to aid programme managers in carrying out and maintaining the mapping of PSPs, as the utility of this exercise goes well beyond just the TB programmes. ${ }^{24}$

Despite the absence of formal engagement with the RNTCP, PPs referred presumptive TB cases to the RNTCP on an ad hoc basis. The current PPM schemes (Table 1) are not conducive for individual PPs to refer patients for diagnosis and treatment to the RNTCP, as limited options are offered through only two available PPM schemes (adherence and slum scheme). The introduction of a scheme aimed at referrals of presumptive TB cases to the RNTCP by individual PPs could address the untapped potential of individual PPs.

GPs and core specialists referred more cases than other specialists and AYUSH. Among GPs, 38\% referred to the RNTCP, contributing to $47 \%$ of PP referrals. This suggests that, as shown in other settings, ${ }^{25-27}$ both GPs as well as core specialists should be targeted in PPM collaborative efforts. AYUSH referred a small number of cases to the RNTCP. Other studies have shown that these are the first contact points for seeking care, especially in rural India, ${ }^{28}$ and authors have stressed the need to engage them in PPM efforts. ${ }^{9}$

The high proportion of presumptive PP-referred TB cases later confirmed as smear-positive cases suggests that PPs tend to make selective referrals to the RNTCP. The reasons for this selective referral, i.e., whether PPs refer only cases with a high index of suspicion of TB instead of referring all presumptive TB cases to the RNTCP, should be further explored.

PSP contribution to the RNTCP is not routinely measured and reported in India. The PPM section of the RNTCP's quarterly reports provides only the absolute number of PSPs involved in any PPM scheme; it does not provide any disaggregated data according to different types of providers. In the absence of clear performance-related indicators for the PPM component, RNTCP staff may circumvent PPM-related activities, as it neither offers any incentives nor contributes to their measurable per- 
formance. ${ }^{29}$ Efforts to strengthen the PPM reporting system by incorporating tools and indicators to measure the PSP contribution should be made.

In conclusion, PPM TB has been implemented for several years in India, with limited success. Nonetheless, there is scope for greater engagement of PPs, who refer a substantial number of cases even without having formal agreements with the RNTCP. The vision of the Government of India is for a 'TB-free India'. To achieve this, the programme has adopted a new strategy in RNTCP Phase III (2012-2017) of universal access to quality diagnosis and treatment for all TB patients by engaging all health care providers. ${ }^{30}$ To achieve this objective, and bearing in mind that a strong public health system is a prerequisite for successful TB control, ${ }^{31}$ it is critically important that PPs are fully involved in the programme. Previous studies in India have highlighted the challenges in involving individual PPs in RNTCP. ${ }^{32}$ It is important to understand the reasons for the absence of formal engagement, if and why PPs prefer informal collaboration, and what strategies would work to build long-term collaboration. Qualitative research into what motivates PPs to collaborate with the RNTCP could contribute to the build-up of this knowledge and to the development of sustainable health system-related interventions to improve referrals of presumptive TB cases from PPs to the RNTCP.

\section{Acknowledgements}

The authors thank the staff of the Revised National Tuberculosis Control Programme for providing the support necessary for conducting this study.

Funding for the study was provided by the Directorate General for Development Cooperation (DGDC), Government of Belgium, Brussels, Belgium. VY is the recipient of a $\mathrm{PhD}$ grant under the DGDC funding.

Conflicts of interest: none declared.

\section{References}

1 World Health Organization. Global tuberculosis control report, 2014. WHO/HTM/TB/2014.08. Geneva, Switzerland: WHO, 2014.

2 Sreeramareddy CT, Qin Z Z, Satyanarayana S, Subbaraman R, Pai M. Delays in diagnosis and treatment of pulmonary tuberculosis in India: a systematic review. Int J Tuberc Lung Dis 2014; 18: 255-266.

3 Kapoor S K, Raman A V, Sachdeva K S, Satyanarayana S. How did the TB patients reach DOTS services in Delhi? A study of patient treatment seeking behavior. PLOS ONE 2012; 7 : e42458.

4 Satyanarayana S, Nair S A, Chadha S S, et al. From where are tuberculosis patients accessing treatment in India? Results from a cross-sectional community based survey of 30 districts. PLOS ONE 2011; 6: e24160.

5 Nimbarte S B, Wagh V, Selokar D. Health seeking behaviour among pulmonary tuberculosis patients in rural part of central India. Int J Biol Med Res 2011; 2: 394-397.

6 Hazarika I. Role of private sector in providing tuberculosis care: evidence from a population-based survey in India. J Glob Infect Dis 2011; 3: 19-24.
7 Udwadia Z F, Pinto L M, Uplekar M W. Tuberculosis management by private practitioners in Mumbai, India: has anything changed in two decades? PLOS ONE 2010; 5: 1-5.

8 Bell C A, Duncan G, Saini B. Knowledge, attitudes and practices of private sector providers of tuberculosis care: a scoping review. Int J Tuberc Lung Dis 2011; 15: 1005-1017.

9 Achanta S, Jaju J, Kumar A M V, et al. Tuberculosis management practices by private practitioners in Andhra Pradesh, India. PLOS ONE 2013; 8: e71119.

10 Kulkarni V, Bajpai A, Palanivel C, et al. Awareness and practices of private medical practitioners regarding pulmonary TB in Mangalore, India. Med Sci 2014; 7; 8-14.

11 Bharaswadkar S, Kanchar A, Thakur N, et al. Tuberculosis management practices of private practitioners in Pune Municipal Corporation, India. PLOS ONE 2014; 9; e97993.

12 Central TB Division. Revised schemes for NGOs and private providers. New Delhi, India: Government of India, 2008.

13 Philip S, Isaakidis P, Sagili K D, Meharunnisa A, Mrithyunjayan S, Kumar A M V. 'They know, they agree, but they don't do'the paradox of tuberculosis case notification by private practitioners in Alappuzha District, Kerala, India. PLOS ONE 2015; 10: $\mathrm{e} 0123286$.

14 Ambe G, Lönnroth K, Dholakia Y, et al. Every provider counts: effect of a comprehensive public-private mix approach for TB control in a large metropolitan area in India. Int J Tubere Lung Dis 2005; 9: 562-568.

15 Murthy K J R, Frieden T R, Yazdani A, Hreshikesh P. Publicprivate partnership in tuberculosis control: experience in Hyderabad, India. Int J Tuberc Lung Dis 2001; 5: 354-359.

16 Floyd K, Arora V K, Murthy K J R, et al. Cost and costeffectiveness of PPM-DOTS for tuberculosis control: evidence from India. Bull World Health Organ 2006; 84: 437-445.

17 Kumar M K A, Dewan P K, Nair P K J, et al. Improved tuberculosis case detection through public-private partnership and laboratory-based surveillance, Kannur District, Kerala, India, 2001-2002. Int J Tuberc Lung Dis 2005; 9: 870-976.

18 Central TB Division. Annual status report of RNTCP 2011. New Delhi, India: Government of India, 2011.

19 Bhatia V. Enhancing private sector contribution to TB care in India. 2010. Geneva, Switzerland: The Global Fund, 2010. http://www.tbpartnershipindia.org/documents/Enhancing private sector contribution to TB care in India.pdf. Accessed January 2016.

20 World Health Organization. Compendium of indicators for monitoring and evaluating national tuberculosis programs. WHO/HTM/TB/2004.344. Geneva, Switzerland: WHO, 2004.

21 Chadha V K, Praseeja P, Gupta J, et al. A descriptive study of tuberculosis case finding in private health care facilities in a South Indian district. Int J Tuberc Lung Dis 2014; 18: 14551458.

22 World Health Organization. Engaging all health care providers. Guidance on implementing public-private mix approaches. WHO/HTM/TB/2006.360. Geneva, Switzerland: WHO, 2006. http: //www.who.int/tb/publications/2006/who_htm_tb_2006_ $360 / \mathrm{en} /$. Accessed January 2016.

23 World Health Organization. Public-private mix for TB care and control. A tool for national situation assessment. WHO/HTM/ TB/2007.391. Geneva, Switzerland: WHO, 2007. http: //apps. who.int/iris/bitstream/10665/69723/1/WHO_HTM_TB_2007. 391_eng.pdf. Accessed January 2016.

24 West Slevin K, Forbes A, Wells W. Public-private mix (PPM) models for the sustainability of successful TB control initiatives. A working meeting co-convened by USAID and the World Bank, in collaboration with the Stop TB Partnership's PPM subgroup, and organized with PATH. Washington DC, USA: US Agency for International Development \& World Bank, 2014. http://www.who.int/tb/careproviders/ppm_tb_demeeting report.pdf Accessed January 2016. 
25 Maung M, Kluge H, Aye T, et al. Private GPs contribute to TB control in Myanmar: evaluation of a PPM initiative in Mandalay Division. Int J Tubere Lung Dis. 2006; 10: 982-987.

26 Pethani A, Zafar M, Khan A A, Rabbani Sana U, Ahmed S, Fatmi Z. Engaging general practitioners in public-private mix tuberculosis DOTS program in an urban area in Pakistan: need for context-specific approach. Asia-Pacific J Public Health 2015; 27: NP984-992.

27 Caminero A, Billo N E. Involving private practitioners and chest physicians in the control of tuberculosis. Tuberculosis 2003; 9792: 148-155.

28 May C, Roth K, Panda P. Non-degree allopathic practitioners as first contact points for acute illness episodes: insights from a qualitative study in rural northern India. BMC Health Serv Res 2014; 14: 182 .
29 Engel N, van Lente H. Organisational innovation and control practices: the case of public-private mix in tuberculosis control in India. Sociol Heal Illn Organ 2014; 36: 917-931.

30 Central TB Division. Universal access to TB care: a practical guide for programme managers. New Delhi, India: Government of India, 2010.

31 Mahendradhata Y, Lambert M-L, Boelaert M, Van der Stuyft P. Engaging the private sector for tuberculosis control: much advocacy on a meagre evidence base. Trop Med Int Health 2007; 12: 315-316.

32 Lal S S, Sahu S, Wares F, Lönnroth K, Chauhan L S, Uplekar M. Intensified scale-up of public-private mix: a systems approach to tuberculosis care and control in India. Int J Tuberc Lung Dis 2011; 15: 97-104. 
CONTEXTE : District de Tumkur, Inde du Sud.

OBJECTIF : Evaluer la participation de médecins privés libéraux (PP) dans le projet d'association public-privé (PPM) dans le cadre du Programme National de lutte contre la Tuberculose révisé (RNTCP) et documenter leur contribution à la recherche de cas de tuberculose (TB) pulmonaire du RNTCP.

SCHÉMA : Les rapports du RNTCP au centre TB du district ont été revus. Les PP ont été cartographiés et leurs références de cas présumés de TB au RNTCP pendant l'année 2011 ont été évaluées grâce aux registres des laboratoires dans des centres de microscopie désignés (DMC).

RÉSULTATS : Aucun des 424 PP ne s'est inscrit à un projet de PPM. Cependant, $22 \%$ ont fait au moins une référence vers un DMC en 2011. Les références des PP ont constitué $15 \%$ des cas présumés de TB examinés dans les DMC. Les PP ont contribué à $23 \%$ des cas de TB à frottis positif détectés. Parmi les références des PP, la proportion des cas à frottis positif confirmés a été élevée $(24 \%)$.

CONCLUSION : Même 15 années après le début du PPM, il y a une absence totale d'engagement formel des PP auprès du RNTCP. Cependant, les PP réfêrent au RNTCP et contribuent à une fraction de la détection des cas de TB. La proportion élevée de cas à frottis positif confirmés suggère que les PP tendent à faire des références sélectives. Davantage d'efforts sont requis pour promouvoir l'engagement des PP auprès du RNTCP.
MARCO DE REFERENCIA: El distrito de Tumkur en el sur de la India.

O BJETIVO: Evaluar la participación de los médicos del sector privado (PP) de salud con fines de lucro en los esquemas de la colaboración publicoprivada (PPM) del Programa Nacional contra la Tuberculosis Revisado (RNTCP) y documentar su contribución a la búsqueda de casos de tuberculosis (TB) pulmonar en el marco del programa.

MÉTODO: Se examinaron las notificaciones al RNTCP en el centro distrital de TB. Se localizaron los PP y se evaluaron sus remisiones de casos con presunción clínica de TB al RNTCP durante el 2011, a partir de los registros de laboratorio de los centros de microscopia designados (DMC).

RESULTADOS: Ninguno de los 424 PP estaba registrado en alguno de los esquemas de la PPM. Sin embargo, el
$22 \%$ remitió como mínimo un paciente a un DMC en el 2011. Las remisiones de los PP representaron el 15\% de los casos con presunción de TB examinados en estos centros. Los PP contribuyeron al 23\% de los casos detectados de TB con baciloscopia positiva. En los casos remitidos por los PP, fue alta la proporción de casos confirmados por baciloscopia ( $24 \%$ ).

CONCLUSIón: Después de 15 años de vigencia de la PPM en la India, se observó una ausencia total de compromiso formal de los PP con el RNTCP. Sin embargo, los PP remiten casos al programa y contribuyen con una proporción de los casos de TB diagnosticados. La alta proporción de casos confirmados por la baciloscopia del esputo indica que los PP tienden a practicar remisiones selectivas. Es necesario reforzar las iniciativas que promueven la adhesión de los PP al RNTCP. 


\section{CHAPTER 2}

Describes TB patients' experiences while they complete the treatment under RNTCP. This forms part of the pre-implementation phase from the patient's perspective. This chapter is based on the paper titled, 'Coping with tuberculosis and directly observed treatment: A qualitative study among patients from South India'. This paper also explores patients' and caretakers' expectations from the programme. 


\title{
Coping with tuberculosis and directly observed treatment: a qualitative study among patients from South India
}

\author{
Vijayashree Yellappa ${ }^{1,2 *} \odot$, Pierre Lefèvre, ${ }^{2}$, Tullia Battaglioli', Devadasan Narayanan' and Patrick Van der Stuyft ${ }^{2,3}$
}

\begin{abstract}
Background: In India, the Revised National TB control programme (RNTCP) offers free diagnosis and treatment for tuberculosis (TB), based on the Directly Observed Treatment Short course (DOTS) strategy. We conducted a qualitative study to explore the experience and consequences of having TB on patients enrolled in DOTS and their caretakers in Tumkur district, located in a southern state of India, Karnataka.

Methods: We conducted 33 in-depth interviews on a purposive sample of TB patients from three groups: (1) patients who reached RNTCP directly on their own and took DOTS at RNTCP; (2) patients who were referred by private practitioners (PPs) to RNTCP and took DOTS at RNTCP; and (3) patients diagnosed by RNTCP and took DOTS from PPs. Data was analyzed using a thematic approach with the support of NVivo9.

Results: The study revealed that TB and DOTS have a large impact on patient's lives, which is often extended to the family and caretakers. The most vulnerable patients faced the most difficulty in accessing and completing DOTS. The family was the main source of support during patient's recovery. Patients residing in rural areas and, taking DOTS from the government facilities had to overcome many barriers to adhere to the DOTS therapy, such as long travelling distance to DOTS centers, inconvenient timings and unfavorable attitude of the RNTCP staff, when compared to patients who took DOTS from PPs. Advantages of taking DOTS from PPs cited by the patients were privacy, flexibility in timings, proximity and more immediate access to care. Patients and their family had to cope with stigmatization and fear and financial hardships that surfaced from TB and DOTS. Young patients living in urban areas were more worried about stigmatisation, than elderly patients living in rural areas. Patients who were referred by PPs experienced more financial problems compared to those who reached RNTCP services directly.

Conclusion: Our study provided useful information about patient's needs and expectations while taking DOTS. The development of mechanisms within RNTCP towards patient centered care is needed to enable patients and caretakers cope with disease condition and adhere to DOTS.
\end{abstract}

Keywords: Tuberculosis, Public-private mix, Coping, Finance, DOTS, Patient-centered care, Nutrition, Private practitioners, India, RNTCP

\footnotetext{
- Correspondence: vijayashreegiphindiaorg

IInstitute of Public Health, \$250, 2nd C Main, 2nd C Cross, Girinagar I Phase, Bangalore 560 085, Karnataka, India

${ }^{2}$ Institute of Tropical Medicine, Nationalestraat, 155, 2000 Antwerp, Belgium Full list of author information is available at the end of the article
}

\section{() Biomed Central}

02016 The Aushorts. Open Access This artide is distributed under the terms of the Creative Commoris Atribution 40

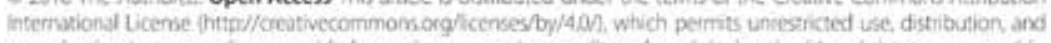
reproduction in any medium, provided you give appropriste credit to the original authoris and the yource, provide a fink to the Creative Commons licerse, and indicate if changes wele made. The Creative Commons Putlic Domain Dedication walver

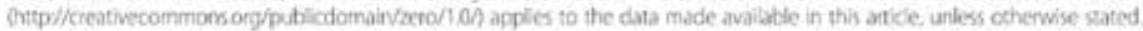




\section{Background}

In India, Tuberculosis (TB) remains a major public health problem and accounted for $24 \%$ of the total 9 million cases reported worldwide in 2013 [1]. Following the World Health Organization (WHO) declaring TB as a 'global emergency' in 1993, the Government of India (GoI) launched the Revised National TB Control Programme (RNTCP) in 1997 based on WHO endorsed Directly Observed Treatment Short course (DOTS) strategy. RNTCP was expanded in a phased manner to achieve complete population coverage by 2006, with an overarching objective to detect at least $70 \%$ of sputum positive pulmonary TB cases and to achieve a cure rate of at least $85 \%$ [2]. The programme follows a 'passive case finding' approach, which assumes that people are able to recognize their symptoms and seek health care. Patients can avail RNTCP services either by directly accessing public health facilities or by being referred by private practitioners (PPs).

RNTCP is implemented mainly through public sector health facilities, which provide free diagnostic and treatment services. However in India, for many TB patients, the first point of contact for health care are PPs [3-6]. Recognizing this, the Government involved PPs through WHO recommended PPM (Public Private Mix)-DOTS strategy $[7,8]$. This partnership enables PPs to refer patients to the RNTCP for either free diagnosis or treatment. Thereafter, the patient can either continue treatment within RNTCP or alternatively PPs themselves can provide DOTS.

Studies from India $[9,10]$ and elsewhere [11-14] have shown that accessing care and adhering with the long course of TB treatment is a complex phenomenon. However, most of these studies used a quantitative approach without further in-depth exploration into the "whys" of the issues. Few studies have used a qualitative approach to explore patient's experiences during the different phases of DOTS treatment [15-18] Moreover, in India there are very few published qualitative studies $[19,20]$ that have captured the experience of patients and their caretakers in obtaining and completing DOTS.

WHO's post 2015 TB strategy states "Integrated, patient centred care", as one of the main component of the strategy [21]. Studies which analysed the reasons for non-adherence to TB treatment in RNTCP have concluded that it stems in a poor match between patients' and programme's needs and priorities [22]. To fulfil the aspiration of 'patient centred care', the programme managers and policy makers will have to give due attention to patient's needs and expectations from the programme. With this background, we conducted this study to explore the experience and consequences of having TB on patients enrolled in DOTS and their caretakers in Tumkur district, located in a southern state of India, Karnataka. The study findings described in this paper are part of a larger study, which investigated how patients navigate through the health system and gain access to RNTCP and the organisational and operational challenges encountered in establishing collaboration between PPs and the RNTCP in Karnataka state.

\section{Methods}

Study design

Qualitative research using in-depth interviews as data collection tool.

\section{Setting}

The study was conducted in Tumkur district (total population 2.716.997), Karnataka state, South India. The district is spread over 10,597 square kilometers, with a population density of about 253 persons per square kilometer. Like elsewhere in India, the district has a pluralistic health system composed of private and public health facilities. As per the RNTCP guidelines, Tumkur district is divided into six TB units, each catering to a population of 500,000 and responsible for program implementation. Under each TB unit, Designated Microscopy Centers (DMCs) provide free sputum microscopy services, each catering to a population of 100,000 . Laboratory registers maintained at DMCs capture patient's information such as name, age, sex, address, source of referral, date of sputum examination, and date of initiation of the TB treatment under the RNTCP. The district has 2,555 DOTS centers that provide free TB drugs and try to ensure treatment completion. The DOTS provider is an observer other than a family member, who supervises and supports the patients in taking their drugs at right doses and intervals. The DOTS provider can be either a public health facility (all of them provide DOTS), a PP, a health worker or any trained community volunteer who is acceptable and accessible to patients and accountable to the health system. If the patient resides far from the health facility, DOTS provision is made closer to the patient's residence through a community health volunteer or a health worker.

\section{Recruitment of study participants}

We conducted a total of 33 in-depth interviews on a purposive sample of new TB patients. Patients were recruited from three different groups that were defined for the purposes of the larger study mentioned above: (1) patients who reached RNTCP directly on their own and took DOTS at RNTCP; (2) patients who were referred by PPs to RNTCP and took DOTS at RNTCP; and (3) patients who were diagnosed by RNTCP and took DOTS from PPs, irrespective of how they initially reached RNTCP. Patients were further stratified for rural $(n=18)$ and urban (15) settings. 
We aimed to interview eight respondents from each category. Patients were shortlisted from RNTCP laboratory registers. Patient characteristics such as, whether they reached RNTCP directly or were referred by a PP or taking DOTS from a PP, duration of the treatment, etc., were cross-checked with RNTCP TB registers and treatment cards. To reduce the recall bias, we selected patients who had completed their treatment in the last three months or who were about to complete the treatment in the next one month at the time of the interview. Although we targeted eight respondents in each category, we interviewed more respondents in the category of 'referred by PPs' $(n=17)$. This is because, in the course of data collection we discovered that some patients who were categorized as 'having reached RNTCP directly' had initially consulted PPs for their illness and were referred by them to the RNTCP.

Of the 33 interviews, 23 participants chose to be interviewed at their residence. Caretakers were present in 21 of these interviews, and helped patients reconstruct their experiences of care seeking. Of the 47 shortlisted patients, 14 patients could not be interviewed, 10 refused to be interviewed, and four had shifted their place.

\section{Data collection}

Data was collected from May 2013 to August 2013. Three interviews guides, one for each category of patients (see Additional files 1,2, and 3), were pilot-tested in February 2013 and fine-tuned accordingly. In order to elicit quality data, interviews were preceded by warm up visits to interviewees by a field coordinator, who shared the information brochure and explained about the research project in the local language, Kannada. Efforts were made to build rapport with patients and an appointment was sought for interviews. Participants were given the choice to choose a date, time and place which was convenient for them. The Principal Investigator (PI) conducted the in-depth interviews in local language Kannada, which lasted between $45 \mathrm{~min}$ to one and half an hour. The main themes that were explored in the interviews were effects and consequences of having TB on individual and caregivers, and their experiences of completing DOTS therapy in RNTCP.

\section{Data analysis}

A preliminary data analysis was conducted simultaneously with data collection. All interviews were audio recorded and transcribed verbatim by professional transcribers. Each transcription was then crosschecked by the principal investigator for accuracy and to include non-verbal communication. We combined deductive and inductive approaches to analyse the data. The deductive approach was based on the research questions and themes related to the experience of TB patients and their caretakers with the organizational and technical aspects of the delivery of DOTS, that were contained in the interview guide (e.g. previous and present experience with TB and DOTS, cost of the treatment, provider related issues, side effects). However, we kept the flexibility of including new themes emerging from the data (inductive approach).

We conducted a thematic analysis [23, 24]. A coding scheme was developed and refined progressively over time (Fig. 1). Segments of the transcripts were classified in the deductive themes. Simultaneously, themes that emerged through scrutinizing the data (inductive) were added. We systematically checked across the transcripts for the consistency of these emerging categories. In later stages of the analysis we explored the relationships between the themes and across different categories of participants to identify patterns in the data. The analysis was conducted with the support of QSR NVivo 9 (QSR International Pvt LTD, Melbourne, Australia).

To increase the internal validity of the analysis, the coding scheme (Fig. 1) was regularly discussed with a sociologist (PL) not involved in data collection and other members of the research team composed of researchers from different backgrounds (e.g. epidemiology, public health).

\section{Ethics}

The study proposal received approval from the Institutional Ethics Committee of the Institute of Public Health, Bangalore and the Institutional Review Board of the Institute of Tropical Medicine, Antwerp and Institutional Ethics Committee of Antwerp University. To avoid any breach in medical confidentiality, the PI after line listing the potential TB patients, shared the list with the district TB officer, who in turn contacted field RNTCP staff to check with patients if they agreed to be interviewed. Following which, RNTCP staff contacted patients over phone and informed them about the interviews. RNTCP staff provided contact details of patients who agreed to participate and helped the PI locate patients using local maps. After explaining the confidentiality and nature of the research project in local language, written informed consent was obtained from all the participants by the PI for their participation in the research. Authorization for audio recording the interviews was also sought. Personal details of the participants were removed from the transcripts to ensure confidentiality and the audio files were anonymised. The NVivo database was password protected and was only accessible to the research team.

\section{Results}

Our results are presented as follows: after summarising the characteristics of the patients, we describe the experience and consequences of having TB on patients enrolled in DOTS and their caretakers. 


\begin{tabular}{|c|c|c|}
\hline Parent Nodes & Child Nodes-1 & Child Nodes-2 \\
\hline \multirow{2}{*}{ Diagnostic practices as perceived by patients } & Sputum examination & \\
\hline & Others & \\
\hline \multirow[t]{3}{*}{ Treatment practices as percelved by patients } & Initiation of treatment & $\begin{array}{l}\text { At private health facility } \\
\text { At gowernment health facility } \\
\text { At medical college }\end{array}$ \\
\hline & Treatment compliance issues & \\
\hline & Non allopathic treatment & \\
\hline \multirow[t]{2}{*}{ Experience of TB Patients with DOTS } & $\begin{array}{l}\text { Experience of patients who took DOTS } \\
\text { from government sector }\end{array}$ & $\begin{array}{l}\text { Organization of services } \\
\text { Side effects } \\
\text { Timing } \\
\text { Travelling } \\
\text { Flexiblity } \\
\text { Attitude of TB hospital staff }\end{array}$ \\
\hline & $\begin{array}{l}\text { Experience of patients who took DOTS } \\
\text { from private practitioner }\end{array}$ & $\begin{array}{l}\text { Reasons for taking DOTS from private practitioner } \\
\text { Side effects } \\
\text { Timing } \\
\text { Travelling } \\
\text { Attitude of the private practitioner } \\
\text { Flexibility }\end{array}$ \\
\hline \multicolumn{3}{|l|}{ Patients recommendations to improve TB care } \\
\hline \multicolumn{3}{|l|}{$\begin{array}{l}\text { Perception of government versus private health } \\
\text { services }\end{array}$} \\
\hline \multirow{2}{*}{ Knowledge about TB } & Source of information & \\
\hline & Prior experlence with TB patients & \\
\hline \multirow[t]{2}{*}{ Perception of TB } & Perception of severity & \\
\hline & Lay aetiologies & $\begin{array}{l}\text { Worm } \\
\text { TB patient } \\
\text { Cigarette and tobacco products } \\
\text { Dust } \\
\text { Unhygienic environment } \\
\text { Eating junk food outside } \\
\text { Heredity } \\
\text { Fever/typhold } \\
\text { Alcohol } \\
\text { Stepping barefoot on sputum or urine of TB patient } \\
\text { Starvation } \\
\text { Poor postnatal care } \\
\text { Through air } \\
\text { Change in food habits } \\
\text { Don't know }\end{array}$ \\
\hline \multirow{2}{*}{ Stigmatisation } & By others & \\
\hline & By self & \\
\hline \multirow{3}{*}{ Coping mechanisms } & Food & \\
\hline & Financial burden & \\
\hline & Family support & \\
\hline \multirow{2}{*}{\multicolumn{3}{|c|}{ Co-morbidity }} \\
\hline & & \\
\hline Patients pathways & Delays & \begin{tabular}{|l} 
Patient \\
Private practitioner \\
Government \\
\end{tabular} \\
\hline \multirow{10}{*}{ Factors influencing patient itineraries } & Role of family and friends & \\
\hline & \begin{tabular}{|l} 
Field health workers \\
\end{tabular} & \\
\hline & Cost & \\
\hline & No relief of symptoms & \\
\hline & Previous diagnostic result & \\
\hline & \begin{tabular}{|l|l} 
Private practitioners suggestion \\
\end{tabular} & \\
\hline & Attitude of government hospital staff & \\
\hline & Trust with providers & \\
\hline & Preference for speciallist & \\
\hline & Others & \\
\hline
\end{tabular}

Fig. 1 Coding Tree 


\section{Participant characteristics}

The average age of participants was 40 with the youngest being 16 and the eldest 70 . They were balanced for gender. Income per month ranged from USD 62 to USD 314. Of the 33 patients, three patients had extra pulmonary TB. All patients were new TB cases, except one and none of them took treatment for multidrug resistant TB. We are unable to provide a table of patient characteristics as the table could lead to conflict in protecting patients' anonymity and confidentiality.

\section{Experience and consequences of having TB on patients enrolled in DOTS and their caretakers}

As explained above, the recruited participants had recently completed their treatment or were by the end of it. Therefore, the experience of the patients with TB and their caretakers is indissolubly linked to their experience with DOTS.

We observed that having TB was not solely an individual patient problem, but in most cases a family issue. Some patients were found more vulnerable than others, because of the relative level of poverty and social support they possessed. Patients who were referred by PPs, patients belonging to lower Hindu caste, and patients with low and middle-income status, tend to speak more about their experiences. This is probably because they had to overcome more barriers to access and complete the DOTS treatment compared to the patients from higher Hindu caste and residing in urban areas.

Our data revealed that the most challenging issues for patients and their caretakers were related to coping with DOTS strategy itself. These challenges can be grouped in two broad categories: 1) organizational and technical aspects of the delivery of the service; and 2) socioeconomic and cultural implications of having a TB patient in the family. The latest encompasses issues that emerged through inductively analysing the data.

\section{Organisational and technical aspects of the delivery of DOTS}

Most of the respondents knew about the importance of completing the treatment and the need for regular follow-ups. They mentioned that the treatment was free under RNTCP and that they had to reach DOTS centres to swallow tablets on alternate days (i.e. Monday, Wednesday and Friday).

DOTS centres open only between 9 am to $1 \mathrm{pm}$, and patients and their caretakers had to make adjustments in their daily routines to reach the health facility. For instance, a family that lived on selling vegetables in the market found it difficult to continue their business, as they had to accompany their mother to a DOTS centre. Hence, they sacrificed certain profit by giving away the vegetables to middlemen in the market. A patient's wife who was working as a sweeper in a theatre was the sole bread earner of the family and she struggled to take care of her husband and simultaneously attend her work. She explains: "In the theatre I worked from morning 11.30 to 12.30 and then took my husband to the hospital by afternoon at I'o clock to swallow tablets. Again from there we would start and reach home by 4.30. And again by 5'o clock, I used to rush to the theatre to work. Like this I managed" [wife of patient who directly reached, $M, R, 48$ years].

Another concern highlighted by some patients was the need to arrange DOTS closer to the patient's residence. Some patients, particularly the daily wage workers, had to forfeit their wages to reach the DOTS centre. Thus, they were left with a choice either to earn their livelihood or to take DOTS therapy. Patients, who were severely weak, who did not have stamina to walk to the DOTS centres and could not afford auto fare to commute to DOTS centre, were adversely affected.

Patients repeatedly expressed that DOTS should be made flexible. In one case a mother, who herself was a DOTS provider was not allowed to give DOTS to her own daughter, who suffered from TB. That particular patient was told by RNTCP staff to visit the hospital, which was $1.5 \mathrm{~km}$ away to take the DOTS. The family wanted to hide the disease status from neighbours, as the patient was not married and as they were anxious that suffering from TB could become a threat to a future marriage. The mother expressed her disappointment and was hurt by RNTCP staff's apathy: "I pleaded them saying "please give it [TB drugs] to me. But they did not give. I felt very bad madam. I have given DOTS to so many patients" [Mother of directly reached patient, F, R, 19 years].

Another patient cautioned, if the programme fails to provide the flexibility in DOTS, patients might quit treatment in the middle. An excerpt: "for people who do daily wage work or go to garment industry, if they reach the workplace late by even $15 \mathrm{~min}$, they will be asked many questions. Will they [owner] consider that they [patients] had been to take the medicines? No... they will be straightaway sent back home. For a patient, his work is more important than the tablet and if the authorities refuse to oblige, he will quit the tablets and proceed for his work" [PP referred, M, U, 32 years].

Patients highlighted the need for improving the attitude of RNTCP staff. They suggested that the program staff should proactively know patient's job profile and prioritise those patients who go for work while giving DOTS. This would enable patients to go back to their work after taking tablets. One patient narrated: "He [another patient who came to take DOTS] is only 35 years old and has two small children. He comes to TB hospital by morning 9.30 and by the time he reaches his house it will be afternoon 2'o clock. He will not have any time to go to work. He is in such a situation that if he does not go for his treatment, he will die and if he does not go for work, his children will die" [PP referred, M, U, 65 years]. 
Almost all patients experienced severe vomiting and giddiness in the initial stages of the treatment and also expressed the need for patient counselling especially in the initial stages of DOTS. Some patients were unhappy that, RNTCP staff attitude was not good in such situations, when patients required the maximum support from them. An excerpt: "If a patient gets vomiting sensation after taking tablets, TB centre staff should take care of the patient. But these people just ask the patient to go away and vomit. So, some patients because of vomiting sensation associated with TB tablets don't take the tablets" [PP referred, M, U, 65 years].

Contrastingly, patients who took DOTS from PPs did not face any such difficulties and all of them were satisfied with DOTS provided by PPs. All the eight patients who received DOTS from PPs resided in urban areas. The common reasons cited for taking DOTS from PPS were the geographical proximity, and flexibility provided by PPs in timings to take DOTS as per the patient's needs. Patients could consult PPs immediately when they experienced some side effects of tablets. One patient who was a sales representative narrated how convenient it was to take DOTS from a PP: "Since I had to leave to my job by 9.00 am, the doctor made arrangements at my convenience. The nurse used to give me tablets early in the morning and I used to take tablets and leave for my job" [Taken DOT from PP, M, U, 50 years].

Female patients were particularly happy about the privacy, which was provided at PP's clinics while taking DOTS. An excerpt; "I would have missed my college, if I had to go in the morning to take tablets from TB hospital. Moreover, there used to be so many people, there [TB hospital]. I used to feel shy. Here [PP's clinic] no one will be there [she was shy while narrating this]. I used to feel comfortable here" [Taken DOT from PP, F, U, 18 years].

Only in two cases, the RNTCP staff took the initiative to link them to the nearest PPs who provided DOTS. In the remaining cases, patients had to negotiate with the staff to look for a DOTS provider closer to their residence "I kept on telling them that it is difficult for me to come here [TB hospital] every day. Then they said, we cannot give drugs to home, but we can give it to a doctor nearby my house. I told them to give tablets there [PP clinic] and I took there for six months continuously" [Taken DOTS from PP, M, U, 43 years].

\section{Socioeconomic and cultural implications of having a TB patient in the family}

The main implications of having TB are fear and stigmatization and an economical burden. Like any disease TB has a cost for the patient. However, inductively analysing our data we found that DOTS as organized in India adds to these problems: the patient becomes visible to others and due to the long duration of the treatment, the families are economically affected.

Patients had mixed feelings when they were diagnosed with TB. They felt sad, shocked and afraid. Patients, especially those whose relatives had died of TB in the past perceived TB as a big problem. About one third of patients felt that TB was not a dangerous disease and that, if the treatment is taken regularly, it could be cured like any other disease. Five of these patients had prior exposure to TB patients who were cured.

Few patients were relieved when they were diagnosed with TB, because they perceived initial symptoms such as coughing blood as more serious or they were afraid to be diagnosed with HIV or cancer. However, these results should be interpreted with caution, because the perception of severity changed with time in some patients due to (a) the counselling from doctors and paramedical staff during the course of treatment (b) moral support provided by friends and relatives and (c) relief of symptoms after starting the treatment.

We observed that almost all the respondents were uncomfortable to use the word "TB". Instead, they referred to it as 'illness'. Most patients did not share their disease status with their relatives or neighbours for the fear of getting excluded or getting bad comments. An excerpt: "I did not want our neighbors to know about this. They [TB staff] said, "we can give the tablets to Anganawadi Centre [DOTS center] or somebody's house near my residence". We refused and said, we will not go anywhere and we will come swallow tablets in the hospital itself" [Directly reached, F, R, 19 years].

The tendency of hiding the disease was more among younger patients. Contrastingly, elderly patients in the rural areas tend to share their difficulties with neighbours and there was a certain degree of social acceptance for these patients. We also observed that patients who had social support in the rural areas were less worried about stigmatisation compared to those who did not have. However, patients residing in urban areas took utmost care not to reveal their disease status to anybody, irrespective of the age group. In rural areas, neighbours usually knew the disease status, as it was difficult to hide from the neighbours as expressed by one patient: "Normally village people will ask lot of questions, whereas in cities no one will ask. When people ask, we have to give reasons as to why we are visiting the hospital daily. Otherwise they will keep asking the same question another 20 times. A situation will come, when we will have to tell everyone whoever asks" [PP referred, F, R, 30 years].

Many patients experienced discrimination in one form or the other. In many instances, patient's relatives stood far from them or did not allow children near them for the fear of contracting the disease. Patients were hurt by 
this discrimination. One patient explains, "when my relatives used to come home to see me, they used to hesitate to even drink water in my home" [PP referred, M, U, 41 years]. However, the same patient defends their behaviour, explaining that TB is a contagious disease and it is quite normal for relatives to fear about it and behave in such a manner. "It is just similar to situations like, if we have dengue in our surroundings, we tend to shut the doors to get rid of the mosquitoes".

Immediate family members were always informed about the disease. In almost all cases, the family was the main source of support during patient's recovery. However, patients imposed certain behaviour on themselves such as self-isolation, keeping their plates and cups separately, not allowing children to come close to them, etc. This behaviour was mostly 'self-induced' because of the fear of spreading the disease to family members and they were extremely cautious about it. Most patients knew that $\mathrm{TB}$ is contagious, and hence closed their mouth while coughing to prevent the spread of the disease to other family members. However, this fear came down eventually when they saw many patients taking DOTS in hospitals. These 'self-induced' behaviours were influenced by previous exposure of patient's parents and their relatives to TB and also induced by health staff suggestions. An excerpt:

"The doctor had informed that the disease would not spread after two months. It will not have the strength. Hence after two moths I went out of house to meet friends" [PP Referred, Male (M), Urban (U), 32 years].

We observed that there was a strong belief among the caretakers that TB patients should be isolated and be provided with separate plates and bed covers. In two cases, it was apparently advised by health staff. Many caretakers believed washing patients clothes in hot water will help in speedy recovery: "clothes should be washed in hot water everyday. Then it will be cured fast" [Directly reached, F, R, 41 years]. One patient was made to stay in a separate house where cattle were kept and food was served there till $4-5$ months after the initiation of treatment. That particular patient narrates how painful this isolation was: "I was feeling very bad that I had to stay away like this. I was thinking why I should live like this, when nobody wants me. But day-by-day I was improving and I was cured. I forgot about all those things" [PP referred, $\mathrm{M}, \mathrm{R}, 50$ years].

Patients and their family struggled to meet the financial burden that surfaced due to TB. All patients suffered from severe symptoms, which incapacitated their ability to work, and found it difficult to earn their living. Major expenditure was towards arranging nutritious food and transportation costs to reach DOTS centre on alternate day. Patients who could afford were mostly residing in the urban areas. They travelled to the DOTS centre in a motorcycle or an auto, mostly accompanied by a caretaker. In India, each trip costs a minimum of Rs.60 [1USD], which is costly considering the fact that patients were unable to earn income during the period. Patients and caretakers from the rural areas took loans to meet the additional expenses from relatives, friends, banks, self-help groups, working place and pledge the jewellery and house. In some instances, parents tried to earn extra money working as maids to take care of their offspring who suffered from TB. Ten patients had taken loans and nine out of them were referred by PPs. They repented on their decision to seek care from PPs instead of directly reaching RNTCP services. Many were still repaying the loan at the time of the interview. An excerpt "If I had gone to government hospital in the first instance itself, we would not have taken this much loan. We would not have been a debtor. God knows when I will be able to clear my loans" [PP referred, M, R, 50 years].

Patients who had severe symptoms were admitted to the hospital and so found it hard to manage their business or secure their job during hospitalisation. One patient, who worked as an electric supervisor, could not stop working for the fear of losing his job. He left for his work from the hospital without informing anybody, with the intravenous cannula intact in his hand. He narrated:

"Even when I was admitted, I had removed the drips [intra venous fluids] and went to work without the doctor's and family's notice. If I did not go, then I would have lost the job and I would not have survived" [PP referred, $M, \mathrm{U}, 41$ years]

Another issue that increased the economic burden of the families was the strong belief that existed among patients and caregivers that it was mandatory to eat nonvegetarian food for speedy and complete recovery from TB. Thus, patients spent substantial amounts of money towards arranging non-vegetarian food. Caretakers on the other hand, made efforts to cope up with extra financial burden to meet this requirement. Villagers went to town to buy fruits and some caregivers managed to cook meat once in three days with lots of financial difficulties and, in some instances neighbours shared mutton dishes when they prepared. Hence, some respondents suggested that the government should provide either banana or egg to the patients along with DOTS.

Two patients, who were Hindus, narrated the experience of eating beef in the anticipation of fast recovery from TB. Eating beef is not allowed in Hindu religion and these two respondents revealed this fact only towards the end of interview with lot of hesitation. Since beef could not be cooked at home, they had to go in search of hotels to eat beef. Every beef meal costed them USD $2-3$. They firmly believed that eating beef helped them in fast recovery and rapid weight gain. They took utmost care not to reveal it to anybody, including the 
family members. "If we eat fish and beef along with tablets properly, we will get cured fast. Only mere tablets and eating the usual food prepared at home will not cure $T B^{\prime \prime}$ [PP referred, $\mathrm{M}, \mathrm{U}, 41$ years].

None of the interviewed patients paid money to avail RNTCP services in the Government hospitals except few who had purchased streptomycin injection from private pharmacies, due to the stock out in government facilities. Almost all the participants expressed gratitude towards government for delivering them free medications, but also suggested that the Government should provide financial assistance to the poor patients who could not earn their livelihood while taking DOTS. Some suggested provision of free shelter and food in the initial two months of the treatment for severely ill, and poor patients, with no social support.

\section{Discussion}

This qualitative study has revealed that TB and DOTS have a large impact on patient's lives, which is often extended to the family and caretakers. The patients who faced the most difficulty in accessing and completing DOTS were the ones who were most vulnerable to the disease because of the relative level of poverty and social support they possessed. In all cases, the family was the main source of support during patient's recovery. Patients residing in rural areas, taking DOTS from the government hospitals had to overcome many barriers to adhere to the DOTS therapy, such as long travelling distance to DOTS centers, inconvenient timings and unfavorable attitude of the RNTCP staff, when compared to patients who took DOTS from PPs in urban area. Patients identified advantages of taking DOTS from PPs such as privacy, flexibility in timings, proximity and more immediate access to care. Patients and their family had to cope with stigmatization and fear and financial hardships that surfaced from TB and DOTS. Patients who were referred by PPs experienced more financial struggle compared to those who reached RNTCP services directly.

Qualitative findings are not generalizable outside study settings. However, our findings are most probably transferable to similar settings in India. Though we took steps to reduce recall bias during participant's recruitment, it could not be ruled out completely. The results described in the present study are solely based on patient's and caregiver's experience and reconstruction of the events that occurred during the treatment of the illness. It could have been useful to complement this information with the perspective of health care providers. Patients were identified from the RNTCP registers and were initially contacted by RNTCP staff over phone to participate in the study. Hence, some respondents could have not wanted to share information regarding program staff that could have jeopardized their relationship with them.
Lienhardt and Ogden have argued that strict and universal application of DOTS can decrease the effectiveness of TB control [25]. Our respondents also expressed concerns for introducing more flexibility in the DOT and suggested to improve the attitude of DOTS providers as reported in other studies [26]. In their study Pranaya Mishra et al. [27] concluded that poor communication between patients and DOTS providers were significantly associated with non-adherence. Our results and the above studies call for strengthening the competences of the health personnel involved in TB care and to improve communication through appropriate training.

In our study, we observed that, patients who took DOTS from PPs were satisfied with the flexibility of the service and the care provided by them. This supports a study finding that patients who took TB treatment outside RNTCP perceived DOTS, as applied in India, to be rigid and intrusive [28]. As mentioned in the background, the majority of TB patients in India seek care from PPs. Hence, every effort to control TB should prioritize the ways to engage with PPs optimally, including the provision of DOTS. In our study, it was found that the programme took the initiative to link only two patients with PPs for DOTS provision. This indicates the need to involve more PPs optimally in the delivery of DOTS, regardless of whether patients seek care in the public or the private sector.

There has been an extensive debate around DOTS versus Self-Administered Therapy (SAT) for TB patients. Results of two randomized control trials have shown that there was little to be gained by DOTS compared with SAT $[29,30]$. A recent study from India also concluded that there was no significant difference between success rate in patients taking DOTS and SAT [31]. Similar findings were obtained in an integrated review of DOTS conducted in Latin-America [32]. Some alternatives for DOTS administration have been also studied and promoted such as community-based DOTS. A systematic review recently conducted by Zhang and colleagues [33] on the impact of community-based DOTS showed that this alternative has improved TB treatment outcomes. Volminck and colleagues, in their systematic review on DOTS for TB control concluded that health care delivery oriented towards the needs and preferences of patients seemed to lead to more satisfied patients [34]. Studies have shown that introducing flexibility in DOTS may indeed allow for more efficient $[35,36]$ and cost effective [37]. Recently, there have been moves in the international TB control landscape. WHO TB control, previously heavily based on DOTS [38] has shifted to the STOP TB Partnership, that focuses on a patientcentered approach to TB care taking into account the needs, perspectives, and individual experiences of people affected by TB [39]. 
It is essential that programme managers recognise that patients strive to adhere to their treatment, but structural barriers prevent them from doing so [40]. Our study resonates largely with the social determinants model of health which put emphasis on the social factors that could affect individuals' ability to seek health care and adhere to a course of treatment [41, 42]. Currently RNTCP is vertically oriented, with more emphasis on programme targets than on the patients [43]. TB advocates have urged for a patient-centered solution to TB control, delivered with dignity and compassion [44]. RNTCP may have to consider this as a priority, if its ambitious goal envisaged in its national strategic plan of providing 'universal access to quality assured free diagnosis and treatment to patients', is to become a reality [2]. Being responsive to the local context, meeting the patient's expectations and needs, and empower patients with the help of the family and caretakers to complete the treatment is a way forward $[45,46]$.

It was our consistent finding that patients always shared their disease condition with their close family members. We further observed that the adherence to treatment was heavily dependent on patient's and caretaker's competing needs of everyday life. Similarly to other studies, social support from spouse and close family members was crucial in adhering to the DOTS therapy [47] and the more the social support, the better the coping with the disease condition [48]. Therefore, the TB programme needs to acknowledge the crucial role of the families and proactively involve them in patient counselling to increase their knowledge and awareness about the disease. A better understanding of TB as a disease condition within the families may improve patient care.

The majority of the respondents did not share their disease status with others, except the immediate family members. A study which assessed the overall TB stigma index in India, Bangladesh, Malawi and Colombia, reported that it was highest in India [49]. Studies from India and neighboring Pakistan reported that women were concerned about rejection by their husbands, harassment by in-laws, and the reduced chances of marriage because of TB [50-52] Similarly, we observed that women respondents in our study were satisfied receiving DOTS from PPs, as it provided them privacy. Patients were discriminated and many of them self-isolated with the fear that they will spread the disease to kith and kins, a finding similar to a qualitative study from Nepal [53]. In our study we found that some field health workers suggested self-isolation to some of the patients. This calls for training of this staff. Overall, all these findings point towards the stigma of TB in the Indian society and the urgent need for the programme to reduce stigma related problems in the TB care and control efforts, by conducting effective awareness programs. However, it is important to point out that the way DOTS is administered in India increases the stigmatization of the disease as shown by our findings.

Our study findings add to the evidence that TB patients in India incur large costs associated with TB illness [5457]. TB had devastating socio-economic impact on our study participants and their families, especially from rural areas and further increased their poverty as reported in other studies [58,59]. This phenomenon typically illustrates the 'medical poverty trap', a situation where expenditure increases and income decreases [60]. We found that arranging for 'nutritious' food during the course of the treatment created a significant financial burden, a finding similar to a study from Zambia which reported on substantive 'special food' expenditures [61].

Our study findings are also in line with the widely recognized phenomenon of 'inverse care law', which explains that those who are in most need of healthcare are least able to access services [62]. Because of long distance and inconvenient timings of DOTS centers, patients had to struggle to reach them on time. Studies have shown that long distance from DOTS centre, having an occupation or other socio-cultural obligations are some of the reasons for patients to discontinue TB treatment $[47,48]$. Another study, which analyzed the factors affecting compliance of patients with DOTS, reported that it is affected by travel expenses and the amount of time that patients must spend travelling to treatment centres. Cost of transport was the reason most frequently given for non-compliance [63]. For improving TB control several authors have stressed that provision of DOTS is insufficient and called for introducing pro poor, equity enhancing measures to address poverty in TB control efforts [64-66]. Initiatives of systematically including TB patients under social protection schemes may prove beneficial in this regard [67].

\section{Conclusion}

Our study provides useful information about patient's needs and expectations regarding the delivery of DOTS in India. We also have flagged the need for developing mechanisms within RNTCP to help patients and their families to cope up with disease condition. The programme should make a paradigm shift, to move to a more patient centred approach.

\section{Abbreviations}

DMCs, Designated Microscopy Centers; DOTS, Directly Observed Treatment Short course; Gol, Government of India; PL, Principal Investigator; PPM, Public Private Mic PPS, Private Practitioners; RNTCP, Revised National TB control programme; SAT, Self-Administered Therapy; TB, Tuberculosis: WHO, World Health Organization

\section{Additional files}

Additional file 1: Interview guide 1. Patients under PPM-DOTS. Questionnaire for in-depth interviews of TB patients under PPM-DOTS (DOCX $29 \mathrm{~kb}$ ) 
Additional file 2: Interview guide 2 Patients referred by PP. Questionnairt for in-depth interviews of TB patients referred by Private Practitioners (PPS) (DOCX $29 \mathrm{~kb}$

Additional file 3: interview guide 3, Patients who reached RINTCP directly. Questionnaire for in-depth interviews of TB patients who have reached DNC directly (not referred by Private Practitioner) (DOCX 29 kb)

\section{Acknowledgements}

Authors would like to thank all study participants for their valuable time and for sharing their experiences.

\section{Funding}

Framework agreement between the institute of Public Health, Bangalore, the Institute of Tropical Medicine, Antwerp and the Belgium Directorate-General for Development Cooperation

\section{Availability of data and materials}

Data supporting our findings are available, Only the data where personal decails are not found can be shared upon request to protect patients' anonymity and confidentiality. Data can be requested from Vijayashree Yellappa, PI of the study. Emailvijayashreegiphindia.

\section{Authors' contributions}

W. PL, PVDS and TB conceived and designed the study. WY collected the data. $\mathrm{VY}$ and PL analysed the data and wrote the first draft of the manuscript. PVDS. TB and ND contributed to revising the manuscript for important intellectual content. All authors read and approved the final manuscript.

\section{Competing interests}

The authors declare that they have no competing interests

\section{Consent for publication}

Not applicable

\section{Ethics approval and consent to participate}

The study proposal received approval from the following institutions: Institutional Review Board of the institute of Tropical Medicine, Antwerp, Numbered 863/13, dated 06.02.2013.

Institutional Ethics Committee of the institute of Public Health, Bangalore, dated 2604.2013

Ethics Committee of Antwerp University, Numbered 13/15/166, dated 1006.2013

After explaining the confidentiality and nature of the research prolect in local language, written informed consent was obtained from all the participants by the PI for their participation in the research. Authorisation for audio recording the interviews was also sought.

\section{Author details}

'Institute of Public Health, \#250, 2nd C Main, 2nd C Cross, Girinagar I Phase Bangalore 560 085, Kamataka, India. Institute of Tropical Medicine, Nationalestraat, 155, 2000 Antwerp, Belgium. 'Public Health Department, Faculty of Medicine, Ghent University, Ghent, Belgium.

Received: 24 November 2015 Accepted: 30 June 2016

\section{Published online: 19 July 2016}

\section{References}

1. World Health organisation. Global TB Report. 2014. http//wwwwhoint/hb publications/global_report/env, Accessed 12 June 2015

2. Sachdeva KS Kumar A. Dewan P. Kumar A. Satyanarayana S. New Vision for Revised National Tuberculosis Control Programme (FNICP): Univetsal access - "Reaching the un-reached": Indian I Med Res. 2012:135(May) $690-4$

3. Sachdeva KS Sytyanaravana S. Dewan PK. Nair SA. Source of Previous Treatment for Re-Treatment TB Cases Registered under the National TB Control Programme. PLoS Med. 2011,6(7).

4. Kapoor SK Raman AV, Sachdeva KS Satyanarayana S. How did the TH patients reach DOTS services in Delhi? A study of patient treatment seeving behavior, Plo5 One, 2012:7/8):e-42458.
5. Hazarika L. Role of private sector in providing tuberculosis care: evidence from a population-based survey in india. $J$ Glob Infect Dis. 2011:3(1):19-24

6. Satyanarayana 5. Nair SA. Chadha SS. Shivashankat R. Sharma G, Yadav S, et al. From where ate tuberculosis patients accessing treatment in india? Results from a cross-sectional community based survey of 30 districts. PLoS One. 2011 .619/e 24160 .

7. Government of India. Fevised Schemes for NCOs and Private Providers 2008. http//Www:tbeindianicirvshowfilephp?ild=3143 Schemes NGO-PP pdf. Accessed on 1 September 2014

8. WHO. Public private mix for IB care and control -A tool kit. WHO. 2010 http//wwww.whaint/tb/careproviders/ppmvPPMTcolwitpdf, Accessed on 10 Dec 2014.

9. Charles N. Thomas B, Watson B. Paja Salthivel M, Chandrasekeran V, Wares $F$. Care seeking behavior of chest symptomatics: a community based study done in South india after the implementation of the ANTCP. PLoS One. 2010;5(9):1-6.

10. Nimbarte SB, Wagh V, Selokat D. Health seeking behaviout among pulmonary tuberculosis patients in rural part of central India. Int $J$ Biol Med Res. 2011:2(1)394-7.

11. Xu B, Diwan VK bogg L Access to tuberculosis care: what did chronic cough patients experience in the way of hedithcare-seeking? Scand J Public Health $2007: 35(4) \cdot 396-402$

12. Uiwaja KN, Alobu I, Nweke CO, Onyerwe EC Healthcare-seeking behavior. treatment delays and its determinants among pulmonary tuberculosis patients in rura Nigetia: a cross-sectional study. BMC Health Serv Rec 2013;13(1)25.

13. Li Y, Ehiri J, Tang S, Li D, Blan Y, Lin H, et al Factors associated with patient and diagnostic delays in Chinese TB patients: a systematic review and metaanalysis. BMC Medicine. 2013;11(1):156.

14. Makwaiwa L, Sheu M, Chiang C. Lin S, Chang PW. Patient and health system delays in the diagnosis and treatment of new and retreatment pulmonary tuberculasis cases in Malawi. BMC Infect Dis. 2014,14,132.

15. Ten Asbroek. AH, Bijisma MW, Malla P. Shrestha 8, Deincij DMU. The road to tuberculosis treatment in rural Nepal: A qualitative assessment of 26 journeys. BMC Health Serv Fies. 2008:8:7.

16. Munro SA, Lewin SA, Smith HI. Patient adherence to tuberculosi treatment: a systematic review of qualitative research int J Tuberc Lung Dis. 2008 . 12(10):1134-8

17. Hane F, Thiam S, Fall AS, Vidal L, Diop AH, Ndir M, et al, Identifying barriers to effective tuberculosis control in Senegal : an anthropological approach. Intern I Tuberc lung Dis. 2007;11539-43.

18. Hasher E, Nocdjikhanow M Sayliddincwa S, Rasulova G, Yuldashova U, Urakova G, et al. Why do tuberculosis patients default in Tashkent City, Uzbekistan? A qualitative study. Int J Tuberc Lung Dis. 2010,14(9):1132-9.

19. Isaskidis P, Rangan S. Pradhan A, Ladomitska J, Reid T, Kelmann K. I cry every day': experiences $\alpha$ patients co-infected with HV and multidrugresistant tuberculosis. Trop Med int Heal. 2013,0x, 00): 1-6.

20. Deshmukh RD, Dhande DJ, Sachdeva KS. Patient and provider reported reasons for lost to follow Up in MDRTB treatment: a qualitative study from a drug resistant TB centre in India. PL.OS One. 2015;10(8) we0135802. 10.1371.

21. World Health organisation. Global strategy and taroets for tuberculosis prevention, care and control after 2015. http//Www, whalint/tib/post2015. tostrategy pdi. Accessed on December 2015

22. Jaiswal A, Singh V, Ooden JA, Porter JDH, Shama PP, Sarin R et al Adherence to tuberculosis treatment : lessons from the urban setting of Delhi India Trop Med int Heal. 2003/3(7) 625-33.

23. Boyatzis AE. Transforming qualitative information thematic analysis and code development. Thousand Oaks CA. Sage, 1998

24. Mq P. Qualitative Research and Evaluation Methods, 3rd ed. Thousand Oaks CA: Sage: 2002.

25. Lienhardt C, Ogden JA. Tuberculosis control in resource-poot countries: Have we reached the limits of the universal paradigm? Trop Med int Heal. $2004,977833-41$,

26. Jain M, Chakole SV, Pawaiya AS, Mehta SC. Knowledge, artitude and Practice of DOIS providers undet ANTCP in Ujain, Madhya Pradesh Nat 1 community Med. 20123344670-4.

27. Mishre P, Hansen HitP, Svend Sabore KKK Adherence to DOIS, Patient Educ Couns. 200653 (1-2):29-37.

28. Pinto LM, Udwadia Zf Private patient percections about a pubiic programme; what do private Indian tuberculosis patients really feel about directly observed treatment? BMC Public Health 2010,10:357. 
29. Walley JD, Khan MA, Nevell JN, Khan MH. Efrectiveness of the direct observation component of DOTS for tuberculosis: A randomised controlled trial in Pakistan. Lancet. 2001,35799257),664-9.

30. Newell N. Baral SC, Pande SE, Bam Ds, Malla P. family-member DOIS and community DOTS for tuberculosis control in Nepal: duster-randomised controlled trial, Lancet. 2006;367(9514),903-9.

31. Parida A, Bairy N. Chogtu B. Magazine R. Vidvasagar S. Comprison of ditectly observed treatment short course with selfadminiatered therapy in pulmonary tuberculosis in uctupi district of southem India. I Cin Diagnostic Pes. 201488: 29-31

32. Zuniga JA An integrated review of directly abserved therapy for tuberculosis in Latin America. Hisp Heal Care int 2015:13:1):46-54. do: $10.1891 / 1540-4153.13 .146$.

33. Znang H, Ehiti J, Yang H, Tang S, LI Y. impact of community based DOT on tuberculosis treatment outcomes a systematic review and meta-analysis. PLoS One. 2016:11:21:0147744.

34. Volmink 1, Gamer P. Directly observed therapy for treating tuberculosis. Cochrane Data base Syst Rev. 2015,75)DOt 10.1002/14651858CD00334

35. Maco \& Sols A. Martinez G Martiny P. Taching tuberculosis patients' Internaloed soclal stigma through patient centred care an intervention study in nural Nicaragua BVC Public Health 20088154 do: $101186 / 1471-2458-8-154$

36. Dermot Maher, Rai Gupta Mubund Upichar, Chris Dye MR. Directly Observed Therapry and Treatment adherence Lancet. 2000,356(9234)

37. Prado TN. Wada N, Guidoni LM, Golub JE, Dietre f, Maciel El Cost effectiveness of community health worker versus home-based quardians for directly observed treatment of tuberculosis in Vitoria, Espirito Santo State Brazil. Cad Saude Publica. 2011;27(5) $944-52$

38. Uplekar M Figueroa Munaz J, Floyd K M U. I FM KF. et al. The Stop TB Strategy: bullding on and enhancing Dois to meet the TB-related Milennium Develooment Gaals. WHO Rep. 2006.22

39. Stop TB Partnershio. The Global Plan to End TE: The Paradiam Shift 2016-2020 2015, Avalable from; htrp//wwwstoptborg/assets/documents/g/cbaijplan GlobalPlanToEndTB TheParadigm5hit 2016-2020_5topTBPartnership pot

40. Lambert ML. Van der Stuytt P. Delays to tubercuicsis treatment shall we continue to blame the victim? Trop Med int Health 2005;10/10;945-6.

41. Hargreaves JR Boccia D. Evans CA. Adato M. Petticrew M. Porter IDH. The cocial determinarns of tubetculosis from evidence to action Am J Public Health. 2011;101(4):554-62

42. Farmer $P$. Social Inequalities and Emerging Infectious Diseases. Emesg Infect D.s. $19962244 \div 259-69$

43. Singh $V$. Significance of foreign funding in developing health programmes In india The case study of RNTCP in the overall context of North-south $c 0$ operation. Heal Adm. 1992:XVit-2]-52-60

44. Pai M Yadar $P$, Anupind $R$. Tuberculosis control needs a complete and patient-centric solution lancet Clob Heal 2014274kel89-90

45. Mucq JCM, Theobuid S, Dick L, Dembele M, An explocation of the concept of cirectly observed treatment for tuberculosis patients ifrom a uniform to a customised aporoach, Int J Tuberc Lung Dis 20037) (April 2002) 103-9

46. Macq J, Torfoss T, Getahun H. Patient empowerment in tuberculosis controi reflecting on past documented experiences. Trop Med int Health. 2007. $12(7) 873-85$

47. Lewis CP. Newell $\mathrm{N}$. Improving tuberculosis care in low income courtries a qualitative study of patients' understanding of "patient suppont" in Nepal. BMC Putic Heaith 70099100

48. Marra CA Marra F, Cox VC Palepu A Ficzgerald IM. Foxtors influencing qual ty of life in pabients with active tuberculosis. Heath Qual Lfe Outcomes. 2004:2:58

49. Somma D. Thomas BE. Karim F, et at, Cender and socio-cutural determinants of TB-related stigma in Bangladesh, indla, Malawi and Colombia. int 1 Tuberc Lung Ds 2008.1277.856-66.

50. Jagganjamma, Kramachandm R, Nirups Charles, V Chandrasekaran, M Muriyandi S. Psycho-socialdysfunction: Perceived and enacted stigma among tuberculosis patients recistered under Revised National Tuberculsois control programme indian I Tuberc. 2008031 (44)

51. Nair DM, George A, Chack KT. Tuberculosis in Bombay : new insights from poor urban patients. $1997 ; 12[1777-85$

52. Whan A. Waley J, Newell J, indad N. Tubecculosis in Pakistar: Socio-cultural constraints and opportunities in treatment. $50 \mathrm{C} \mathrm{SCi} \mathrm{Med.} \mathrm{2000,5002r-247-5.4}$

53. Baral SC Kark DK. Nowel iN Cuses of sticma and digcriminticn associated with tuberculosis in Nepat a qualitative study. ENC Public Heaith. 2007;7211.
54. Tanimura $\mathrm{T}$, Jaramillo E, Weil D, Raviglione M, Loonoth K Financial burden for tuberculosis patients in low- and midole income countries: a systematic review. Eur Respir $2.2014,43(6): 1763-75$.

55. Pantoja A Floyd K. Unnilgishnan KP, fiendin R. Padma MR Lal S5, et a. Fconomic evaluation of public-private mix for tuberculosis care and control India. Part L Socio-economic profile and costs among tuberculosis patients int J TB Lung. 2009,13698-704.

56. Floyd K Arora VK. Murthy KR, Lonnroth K Singla N, Akbar Y, et al. Cost and cost-effectiveriess of PPM-DOTS for tuberculosis control : evidence from indla. Bull World Health Organ. 200684i6)437-45

57. John KR. Daley P. Kincler N, Oxlade Q. Nenzies D. Costs incurred by patients with pumonary tuberculosis in nutd india Intern I Tuberc lung Dis 2009. 13/10):1281-7.

58. Rajeswari R. Balasubramanian R. Munivand $M$. Geetharamani S. Thresa $X$, Venkatesan P. Socoeconomic impact of tuberculosis on patients and family in ind a. Intern J Tuberc lung Dis. 1999,3(10) $669-7$ )

59 Mauch V. Woods N. Kinbi B. Kipruto H. Stienei L. Kinkenberg E. Assessing access barriers to tuberculosis care with the tool to Estimate Patients' Costs pilot results from two districts in Kenya. BMC Public Health. 2011:1101t43

60. Dahlgren G. Whitehead M. European strateqies for tacking social inecuities in heath Levelling up Part 2 World Heal Organ, 2006:3:1-140

61. Needham DM, Bowman D, Foster SD, Godfey-Faussett P. Patient care secking bartiers and tuberculosis programme reform: a qualitative study. Heath Policy. 2004,67(1) 93-106.

62. Hart JT. The inverse Care Law, Lancet. 1971;1(7696) 405-12

63. Boyle S.OO, Power II, Ibrahim MY, Watson JP. Factors affecting patient complance with ant tuberculosis chemotherapy using the directly obsenved treatment, short-course strategy. Int I Tubetc Lung Dis 20025307-12

64 Baneri D, Virchow R A social science approoch to strenghening India's national tuberculsoís programme indian ITQ 1993;40(61),26-8.

65. Worid Health Oeganization. Addressing Poverty in TB Control-Options for National Ta Control Programmes. Worid Health, 200554-80.

66. Grede $N$, Claros IM, de Fee S. Bioern M. is there a need to mitigate the social and financlal consequences of tuberculosis at the individual and househoid level? ADS Behav. 2014:18542-53

67. Kamineni W, Wison N, Das A. Satyanarayana S, Chadha S, Singh Sachdeva $\mathrm{K}$ et al. Addressing poverty throuch discase control proarammes examples from Tuberculosis control in india int / Equity Health, 2012:11017: 7

Submit your next manuscript to BioMed Central and we will help you at every step:

- We accept pre-submission inquiries

- Our selector tool helps you to find the most relevant journal

- We provide round the clock customer support

- Convenient online submissior

- Thorough peer review

- Inclusion in PubMed and all major indexing services

- Maximum visibility for your rescarch

Submit your manuscript at www.biomedcentral.com/submit

C) Blomed Centra 


\section{CHAPTER 3}

This chapter reflects the pre-implementation phase from PP's perspective. This chapter is based on the paper titled, 'Patients pathways to tuberculosis diagnosis and treatment in a fragmented health system: A qualitative study from a South Indian district'. This chapter describes how TB patients had to resort to multiple contacts due to the limitations of PPs in diagnosing TB and/or failure to direct patients to RNTCP. This chapter then sheds light on PP's preferred modes of collaboration with RNTCP. 


\title{
Patients pathways to tuberculosis diagnosis and treatment in a fragmented health system: a qualitative study from a south Indian district
}

\author{
Vijayashree Yellappa ${ }^{1,2^{*}} \odot$, Pierre Lefèvre ${ }^{2}$, Tullia Battaglioli ${ }^{2}$, Narayanan Devadasan' and Patrick Van der Stuyftt ${ }^{2,3}$
}

\begin{abstract}
Background: India's Revised National Tuberculosis (TB) Control Programme (RNTCP) offers free TB diagnosis and treatment. But more than 50\% of TB patients seek care from private practitioners (PPs), where TB is managed sub-optimally. In India, there is dearth of studies capturing experiences of TB patients when they navigate through health facilities to seek care. Also, there is less information available on how PPs make decisions to refer TB cases to RNTCP. We conducted this study to understand the factors influencing TB patient's therapeutic itineraries to RNTCP and PP's cross referral practices linked to RNTCP.

Methods: We conducted in-depth interviews on a purposive sample of 33 TB patients and 38 PPs. Patients were categorised into three groups: those who reached RNTCP directly, those who were referred by PPs to RNTCP and patients who took DOT from PPs. We assessed patient's experiences in each category and documented their journey from initial symptoms until they reached RNTCP, where they were diagnosed and started on treatment. PPs were categorised into three groups based on their TB case referrals to RNTCP: actively-referring, minimally-referring and non-referring.

Results: Patients had limited awareness about TB. Patients switched from one provider to the other, since their symptoms were not relieved. A first group of patients, self-medicated by purchasing get rid drugs from private chemists over the counter, before seeking care. A second group sought care from government facilities and had simple itineraries. A third group who sought care from PPs, switched concurrently and/or iteratively from public and private providers in search for relief of symptoms causing important diagnostic delays. Eventually all patients reached RNTCP, diagnosed and started on treatment. PP's cross-referral practices were influenced by patient's paying capacity, familiarity with RNTCP, kickbacks from private labs and chemists, and even to get rid of TB patients. These trade-offs by PPs complicated patient's itineraries to RNTCP.

Conclusions: India aims to achieve universal health care for TB. Our study findings help RNTCP to develop initiatives to promote early detection of TB, by involving PPs and private chemists and establish effective referral systems from private sectors to RNTCP.
\end{abstract}

Keywords: Tuberculosis, Private practitioners, Referral practices, Therapeutic itinerary, Kickbacks, RNTCP, PPM, Private pharmacists, India

\footnotetext{
- Correspondence: vijayashreegiphindia.org

'Institute of Public Health, $\$ 250$, 2nd C Main, 2nd 'C' Cross:, Girinagar I Phase,

Bangalore, Karnataka 560 085, India

${ }^{2}$ Institute of Tropical Medicine, Nationalestraat, 155, 2000 Antwerp, Belgium

Full list of author information is available at the end of the article
} 


\section{Background}

In India, Tuberculosis (TB) is a major public health problem, carrying one fourth of the global burden of TB [1]. Government of India launched Revised National TB Control Programme (RNTCP) in 1997 based on World Health Organisation (WHO) endorsed Directly Observed Treatment Strategy (DOT). The programme follows a 'passive case finding' approach, which assumes that people are able to recognise their symptoms and access health care in time. RNTCP is implemented mainly through government health facilities, which provide quality assured free diagnosis and treatment. TB patients can avail RNTCP services either accessing directly or being referred by Private Practitioners (PPs).

In spite of the availability of free public services, more than $50 \%$ of patients are estimated to seek care in the private health sector $[2,3]$. But, studies have shown that the TB management practices in private sector is suboptimal [4, 5]. Recognising the critical importance of PPs, RNTCP is involving them through Public Private Mix (PPM) strategy [6]. PPs can refer TB patients to RNTCP either for free diagnosis and/or treatment. However, PP's involvement in RNTCP is meagre [7], despite the promising results of PPM strategy $[8,9]$.

Although RNTCP is successful in terms of increase in the proportion of patients treated, many TB cases are missed by RNTCP. In fact India tops the list of ten countries that account for $74 \%$ (2.4 million) of the estimated missed cases globally [1]. 'Missed cases' are those cases which are neither detected nor notified to RNTCP. This can be attributed to either patients delay in accessing healthcare or health system delays [10] or to PPs' reluctance to notify cases to RNTCP [11]. Several studies in India have analysed health seeking behaviour of TB patients and the factors determining their decision-making $[12,13]$. Much of the studies assessing diagnostic and treatment delays have aimed to quantify the delays [14, 15]. The determinants of these delays are much more complex and require a comprehensive understanding of patients' journey from the onset of symptoms until they are successfully treated. But, there is scant published research in India that analyses TB patients experiences holistically, as they navigate through health facilities. Further, it is also critical that PP's TB management practices, their cross-referral practices linked to RNTCP and patients' personal beliefs be comprehended and corroborated.

With this background, we conducted this study among TB patients taking treatment in the RNTCP and PPs providing TB care in Tumkur district, a south Indian state. We aimed to understand patient's therapeutic itineraries to RNTCP, factors influencing the itineraries and corroborated with PP's cross referral practices linked to RNTCP.

Data presented in this paper were collected as part of a larger study, which investigated the operational challenges in establishing the collaboration between PPs and RNTCP in Karnataka state.

\section{Methods \\ Study design}

Qualitative research using in-depth interviews as data collection tool.

\section{Study setting}

Tumkur district (population of 2.7 million, spread over $10,597 \mathrm{~km}^{2}$ ) has pluralistic health system composed of private and public health facilities. Public sector includes a district hospital, ten sub-district hospitals and 146 primary health centres. Private health sector is heterogeneous comprising of highly specialised PPs to informal providers, who practice modern medicine without any formal training. Retail private chemists often dispense drugs over-the counter. Sub-districts are responsible for TB programme implementation. Under each sub-district, there are designated microscopy centres, which provide free sputum smear microscopic services. District has 2555 DOT (Directly Observed Treatment) centers that provide free RNTCP TB drugs and ensure treatment completion. DOT provider can be a public health facility (all of them provide DOT), a PP, a health worker or any trained community volunteer who is acceptable and accessible to patients. Structure and functioning of RNTCP is elaborated elsewhere [16].

\section{Study participants and sampling}

We conducted in-depth interviews with a purposive sample of TB patients aged 15 years and above $(n=33)$. Patients were stratified for rural $(n=18)$ and urban $(n=15)$ settings. Patients started on the RNTCP treatment were shortlisted from RNTCP laboratory registers, and categorised depending on how they reached RNTCP: (i) those who reached RNTCP directly by their own without any referral (ii) those referred by PPs to RNTCP for diagnosis and/or treatment and (iii) those who were diagnosed by RNTCP and referred to PPs for DOT. Patient details were collected from patient TB treatment cards and TB registers. Patients who had completed their treatment in the last 3 months or who were about to complete the treatment in the next 1 month at the time of interviews were selected to reduce recall bias. We aimed to interview eight respondents from each category to reflect the typical experiences of patients based on the principle of data saturation. Although we targeted eight respondents in each category, we interviewed more respondents in the category of 'referred by PPs' ( $n=17)$. This is because, during the course of some interviews, we discovered that some patients who were categorised as "having reached RNTCP directly' had initially consulted PPs and were referred by them to RNTCP. 
PPs $(n=38)$ were selected purposively from the list ( $n=198$ ) maintained at the district TB centre. We collected number of presumptive TB case referrals made by PP's to RNTCP for the year 2012 from RNTCP laboratory registers, and categorised PPs into three groups based on the referrals into: (i) actively-referring PP; who had referred 12 or more than 12 cases $(n=17)$ (ii) minimally-referring PP; who had referred at least one, but less than 12 cases $(n=8)$ and (iii) not-referring PP; who had not referred any case $(n=13)$. Though, we considered all the 19 actively-referring PPs, we could interview 17 of them, since two of them were out of town during the study period. Efforts were made to match non-referring and minimally-referring PPs with actively-referring PPs for age, qualification, patient load and location of practice.

\section{Data collection}

Data were collected from May 2013 to August-2013. Six interview guides, one for each category of patients and PPs were pilot-tested in February 2013 and fine-tuned. Interviews were preceded by warm up visits to interviewees by the field co-ordinator and the principal investigator (PI) of the study (VY). The field coordinator shared the information brochure with patients in local language, Kannada. Similarly, the PI visited PPs and shared the information brochure in English. During these visits, an appointment was sought for interviews. All interviews were conducted by the PI and they were audio recorded.

Interviews with patients were conducted in Kannada that lasted 45 to $90 \mathrm{~min}$. Of the 33 interviews, 23 participants chose to be interviewed at their residence and the remaining at DOT centres and near their working place. Family members were present in 21 of these interviews and helped patients to display laboratory reports and medical prescriptions wherever available. Of the 47 shortlisted patients, 10 refused to be interviewed, and four had moved out.

PP's interviews were conducted in their health facilities, on an average we had to make three attempts to get their appointments. Interviews with PPs were mix of English and Kannada, which lasted 45 to $80 \mathrm{~min}$. Interviews were transcribed verbatim by professional transcribers from the digital version. Each transcription was then crosschecked by the PI for accuracy. In addition, the note taker documented the main points raised, setting descriptions and any relevant informal conversations happened before and after the interviews.

\section{Data analysis}

To maintain the confidentiality, personal details of participants were removed and audio files were anonymized. Data were analysed using a combination of deductive and inductive approaches. The deductive approach was based on the research questions, which primarily aimed to examine the factors influencing TB patient's therapeutic itineraries to RNTCP and understand PP's cross-referral practices to RNTCP. VY and PL devised a coding scheme jointly. Each transcript along with the field summary notes was coded by using NVIVO software version 9 (QSR International Pvt. LTD, Melbourne, Australia). These initial codes were tested on a handful of interviews, which were then refined and organised at a broader conceptual level into themes by grouping them together $[17,18]$. We considered the flexibility of including new themes emerging from the data (inductive approach). This approach helped us to identify relationships and corroborate PP's and patient's views. We then conducted thematic analysis, and explored the relationships between and across the themes across different categories of participants [19]. Memos were written on patient's navigation to RNTCP and PP's cross referrals practices and the data were corroborated. To increase the internal validity of the analysis, the coding scheme was regularly discussed with a sociologist (PL) not involved in data collection and other members of the research team.

The analysis indicated the challenges that the patients face as they navigate through the fragmented health care settings; patient's making sense of the symptoms and the disease, patient's making decision to seek care, patient therapeutic itineraries until they were diagnosed with TB, PP's TB management practices and how it is perceived by patients, how PP's make a decision to refer cases to RNTCP and how it influences patient's itineraries.

\section{Results}

After summarising the socio-demographic characteristics of participants, we present the findings under the following major themes: first we present patients reported initial symptoms and their TB awareness. Then we illustrate patient's itineraries from the onset of symptoms to TB diagnosis at RNTCP's microscopy centres. Later we proceed to elaborate the factors that influence patient's itineraries including PP'S TB management and their cross referral practices linked to RNTCP. In the following result section we elaborate these themes.

\section{Participant's characteristics}

Average age of patients was 42 years (range 18 to 70 years). Gender was equally represented. Reported income per month ranged from USD 46 to 229 . All patients had pulmonary TB, expect three who had extra pulmonary TB and none of them took treatment for multidrug resistant TB. Average duration of PPs' experience was 26 years (range 7 to 39 years). All PPs except three were male; 23 were from rural area and 15 from urban; 32 practiced allopathy (17 general practitioners and 15 specialists) and six were informal providers. 
Patients reported initial symptoms and their TB awareness Excluding patients who had extra pulmonary TB, most frequently reported symptoms were cough associated with fever, tiredness and weight loss. Two patients spoke about coughing blood. Patient's, awareness about TB seems to be limited to the fact that the disease is transmitted by cough and to be influenced by previous exposure of their relatives to TB. A number of patients stated quotations very similar to this one: "No. I was not aware of anything about TB. I never knew anything about it. Nobody from my family or neighbours had this" [Man, Rural, PP Referred]. Patients cited the following reasons as sources of TB transmission: getting exposed to TB patients and dust, living in unhygienic environment, alcohol consumption, smoking and eating junk food on roadside. Men mostly advanced the latter reasons. There were some other factors seldom cited by patients from rural settings such as starving, typhoid fever, walking on sputum or urine of a TB patient and even worms. People who had co-morbid conditions such as HIV or asthma, perceived TB as a lesser problem, because they thought TB could be cured, but not the former conditions.

\section{Patient's therapeutic itineraries}

From the onset of symptoms, two main courses of action emerge from the data. In Fig. 1; Therapeutic itineraries of TB patients, we illustrate the navigation of 33 patients through various health care facilities from the onset of symptoms to TB diagnosis at RNTCP's designated microscopy centres.

The first group of patients $(n=13)$ self-medicated by purchasing symptomatic drugs over the counter from nearby retail private chemists for a period varying from 2 weeks to seven and a half months. Two patients from this category, patient-D4 and patient-R2 encountered the longest delays before getting diagnosed with TB, up to 8 months and 11 months respectively (Fig. 1).

Of the eight patients from the 'directly reached category,' three patients (D1, D3 and D5) sought care from the government facilities directly and they were diagnosed there with no delay. Remaining five patients sought care from private chemists initially, by purchasing symptomatic drugs over-the counter. Since the symptoms did not subside, they accessed RNTCP by themselves based on the following factors: (i) suggestions by the government field health workers, (ii) familiarity with government hospitals, and (iii) having had health personnel among their relatives. In general, this category of patients who reached RNTCP directly had simple itineraries, with least number of providers visited compared to the patients who first sought care from PPs (Fig. 1).

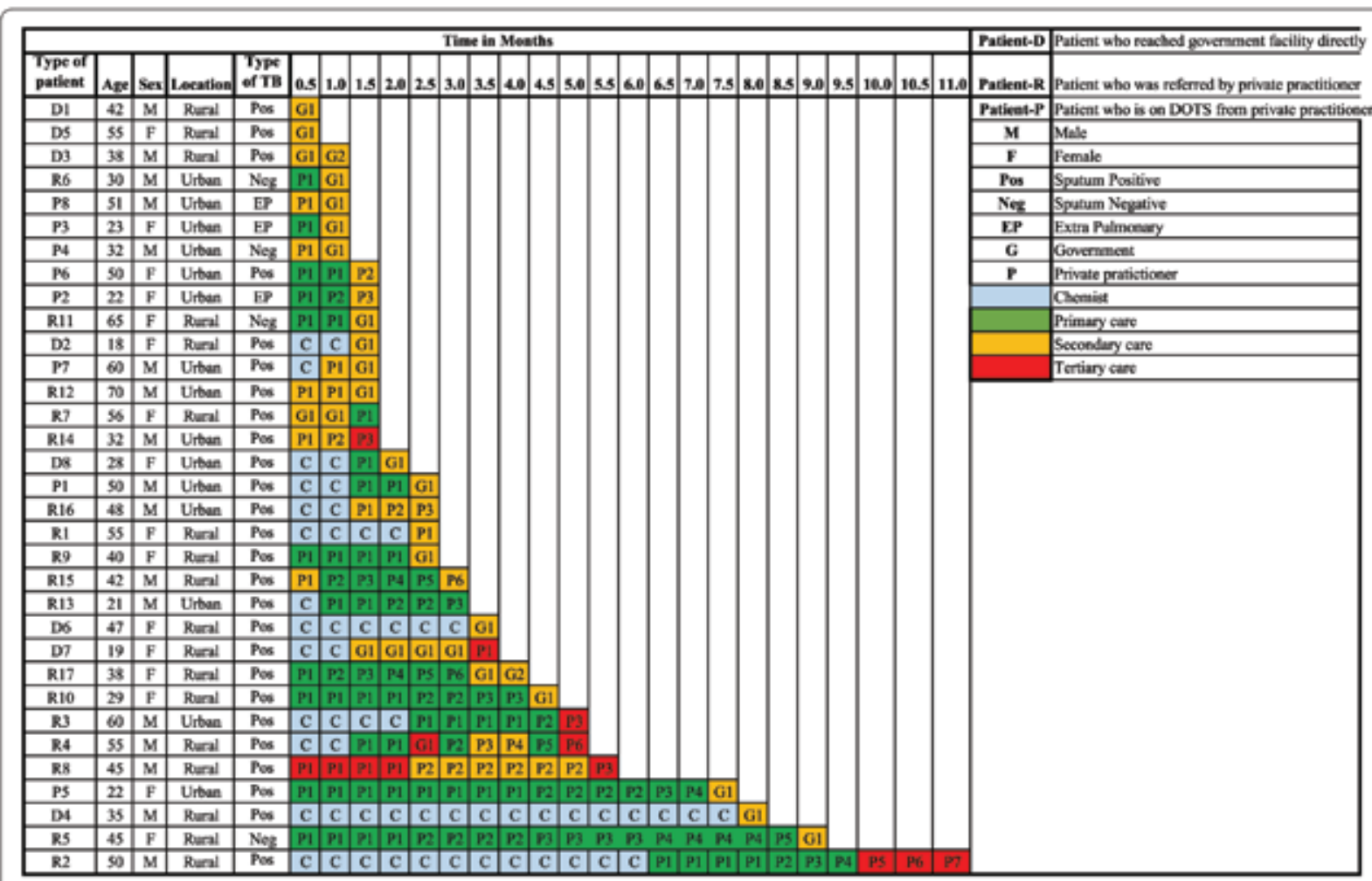

Fig. 1 Therapeutic itineraries of tuberculosis patients 
A third group, who sought care from PPs $(n=17)$, switched concurrently and/or iteratively from public and private providers in search for relief of symptoms causing important diagnostic delays (Fig. 1). Eventually all patients reached RNTCP and were started on treatment. Of the 17 PP referred cases, one patient had visited the government facility initially. Since patient's symptoms were not relieved there, he sought care from a PP. He expressed his dissatisfaction about the poor services offered at government hospital. This concern was also raised by three PP referred patients (R4, R7, R17):

\section{"Government doctor did not show any interest, neither he responded to my questions. They never spoke to me at all. We went there 1-3 days and became fed-up. Even the $4^{\text {th }}$ day they did not say anything. They asked me to go here and there. It was really a horrible experience to run around there. So, finally we decided and went to private" [Man, Rural, PP referred].}

More than half of the respondents approached primary level health facilities in the initial course of illness (either private or public). However, except one patient (R7), final diagnosis of TB was made at either secondary or tertiary level facilities after much itinerancy (Fig. 1).

Factors influencing patient therapeutic itineraries If we consider all patients, three factors played an important role influencing patient itineraries; (i) influence of significant others, (ii) PP's TB management practices, (iii) PP's cross referral practices linked to RNTCP (iv) provision of kickbacks and (v) patient's search for relief.

\section{Influence of significant others and preference for particular providers}

More than half of the patients $(n=16)$, while discussing their therapeutic itineraries spoke about the role of the family, relatives, friends and even neighbours at some point of time. These interventions took various forms, ranging from suggesting the patient to go to a particular hospital or to change the provider, or even 'force' the patient to seek care. A quote:

"She [Daughter] said that the symptoms sound like TB. So, we should go and get a check-up" [Woman, Rural, Directly Reached].

We observed that the trust in family doctors played an important role in at least one third of patients in choosing a particular doctor. Patients esteemed these providers; "He is our family doctor. Whatever happens in our family we will get treatment from him" [Woman, Urban, Patient taking DOT from PP].

\section{Provider's TB management practices}

TB management practices of PPs played an important role in influencing patient's itineraries to RNTCP.

Low index of suspicion of TB among PPs Indirect evidence of low index of suspicion of TB among PPs can be deduced from patients' narratives. Some patients were misdiagnosed and treated for typhoid and dust allergy for a period ranging from 1 week to 3 months with no relief of symptoms. Patients were unhappy that PPs were unable to detect TB in the first instance and that they were made to undergo unnecessary tests. An excerpt:

"He [PP] had told me to take injections daily and I was taking it as advised. But he did not tell me anything. He kept on saying it is typhoid. We told him that sputum is coming while coughing. But he said, it will happen like this even for typhoid also" [Woman, Rural, PP referred].

This was conceded by some PPs, who expressed how increasingly it is becoming difficult to diagnose TB because of varied clinical presentations associated with HIV and diabetes. PPs highlighted the difficulties they face to diagnose extra-pulmonary TB and sputum negative pulmonary TB cases.

PP's TB management practices PPs used a battery of investigations to diagnose TB, such as chest X-ray, blood tests, sputum examination, and Mantoux test by order of preference. Chest X-ray was most preferred diagnostic tool, since PP's perceived it to be rapid and patient friendly. An excerpt:

" $X$-ray is fast. We get the result in 10-15 minutes and immediately we can take some decisions. You know... we do not want to miss any patients (smiles)" [Actively-Referring PP, Rural, Specialist].

Majority of PPs tend to reject X-rays taken at the government hospitals, as they perceived them to be of poor quality. Thus, patients were asked to repeat the chest Xrays in private labs, which meant double expenditure and investment of time for patients. A quote:

"I don't trust government hospital for X-ray. Quality of $X$-ray is not good there. $X$-ray technician there [Government hospital] is not efficient. Only if patients refuse to go to private $X$-ray facility, we will send them to government for X-ray" [Actively-Referring PP, Rural, General Practitioner].

Contrastingly, all the actively-referring PPs preferred government hospitals for sputum microscopy. They 
placed great trust on the technical competency of government laboratory technicians and believed that the technicians are regularly trained as compared to private ones. They listed several advantages of ordering sputum examination compared to other TB tests: being specific for TB, easier to communicate the TB diagnosis to patients when sputum results are positive and helps in monitoring the disease condition. This group of PPs were familiar about RNTCP and referred most of their cases to RNTCP for sputum examination. In some instances when patients refused to go there, because of perceived low quality of care, PPs counselled them. A quote:

"RNTCP lab technicians are well trained. They always give good results. When some patients insist that they will get the tests done from private labs, I tell them. "There [private lab] they [lab technicians] will not do it well". We insist them [patients] to go to government only. I never send my patients to private labs for sputum AFB [Acid Fast Bacilli]" [Actively-Referring PP, Rural, General Practitioner].

As far as the TB treatment practices were concerned, actively-referring PPs preferred to refer cases to the RNTCP. In instances where patients refused RNTCP treatment, they were started on private treatment with daily regimen, four anti-TB drugs for initial 2 months, followed by two anti-TB drugs for 4 months. Minimallyinvolved and non-involved PPs either treated TB patients sub-optimally for 3 months or over-treated for a year and a half, with inappropriate regimen. Antibiotic Oflaxacin was mostly combined with anti-TB treatment regimens. All PPs except one (Non-referring PP-4) acknowledged that, patients tend to default after 2 months of private treatment once the symptoms subside. They also stated that they cannot follow up patients to ensure treatment completion. Informal providers also treated TB patients with inappropriate regimen for 3-4 months. Their TB prescription knowledge was mainly based on medical representatives visits and the drugs they propose. When patient's symptoms did not subside, they tend to refer them to specialists located in district head quarter.

PP's cross referral practices linked to RNTCP The decision to refer patient to RNTCP was solely dependent on PPs' discretion. Most of the actively-referring PPs were familiar with the RNTCP and preferred to refer their cases to RNTCP. Some of them even believed that, it was their duty to co-operate with government, since most of them had received subsidised medical education from the government. Others expressed that referring patients to RNTCP was a social service. A quote:
"My patients are poor. They cannot afford private treatment. So I refer them to TB hospital. I feel it is a social service that I am doing" [Actively referring PP, Urban, Specialist].

However, minimally-referring and non-referring PPs' decision to refer patients to the RNTCP was solely dependent on patient's paying capacity. They referred only such patients to the RNTCP, whom they perceived of incapable of paying.

"I will be knowing their [patient's] financial condition. I can make out whether they are affordable or not. If they are unaffordable, I will ask them to get the sputum test done in government" [Non-Referring PP, Urban, Specialist].

Contrastingly, they retained such patients who had the capacity to pay for private treatment. We observed that some minimally-referring PPs referred patients to the RNTCP to get rid of them. They perceived TB patients as nuisance, since TB patients bothered them frequently with small ailments and repeated counselling consumed lot of their time. In some instances, when PPs sensed that they could not ensure patient's compliance to treatment, they referred such patients to RNTCP. A quote:

"I do not have time to sit and explain to the patients, about what is TB and all. This communication is very time consuming. We should finish our consultancy in 3-4 sentences. If I keep giving health education, I will have to close my clinic (laughs). So I refer them to government, so that they don't come back to me again" [Minimally- Referring PP, Urban, General Practitioner].

Informal providers' decision to refer patients to the RNTCP was dependent mostly on the infrastructure they possessed. PPs having small clinics referred their cases to the RNTCP because, of lack of knowledge about TB, fear of spread of infection in their clinic and availability of free treatment in the RNTCP. But the informal PPs owning nursing homes preferred to retain patients and they treated them for three to 4 months with private TB drugs.

We also observed a tendency among minimallyreferring and non-referring PPs to put the blame on the patients or the poor functioning of government hospitals, as a reason for patient's unwillingness to go to RNTCP as per their advice. A quote:

\footnotetext{
“Government doctors do not treat patients well. The relationship between the patient and private practitioner is better compared to that of the government doctor and patient. That is the basic
} 
principle you should understand" [Non-Referring PP, Rural, General Practitioner].

As a result of the above-described PP's trade-offs, patient's itineraries became complex. It was our consistent finding that once patients reached the RNTCP, treatment was started within a week in all cases.

\section{Provision of kickbacks to PPs}

When PPs were asked whether they receive any kickbacks from private laboratories for referring patients there, majority of PPs denied receiving kickbacks and some specialists were even offended by the question. However on further probing, few $(n=8)$ revealed that private laboratories gave kickback of around 5-10\%, which they perceived to be meagre compared to the big labs located in big cities, where it could be as high as $30-40 \%$. An excerpt:

"Nothing... they are [private labs] just giving it [kickbacks] for the sake of it. It is not like Bangalore where they give $30 \%$ or $40 \%$. Here [Tumkur city] they give 5 or $10 \%$... it is nothing" [Minimally-Referring PP, Urban, Specialist].

It appeared that the phenomenon of kickbacks was a norm in the private health sector and was available even to unqualified informal providers. An excerpt:

"That is usual no madam...For X-ray, they [private labs] give 120 rupees [1.8USD] per patient referred. For sputum examination, they charge 50-60 rupee [1USD] per patient. So we hardly get anything there. Pharmacies... not really. They usually give us some gifts. You know how it works (laughs)" [Non-Referring PP, Rural, Informal provider].

\section{Patient's search for relief}

If we consider all participants, except four patients (patients D1, D3, D5 and R7), all other patient's first encounter was with either private chemists or PPs. Since symptoms were not resolved, patients were forced to switch from one provider to another, concurrently and/or iteratively from public and private providers, leading to important diagnostic and treatment delays. An excerpt:

"First they [PPs] told me to get X-ray, then they told me that there was no problem. I threw that report... I kept on visiting doctors and getting tested. I even got my blood and urine tested, but I was informed that there was no problem. I threw those reports too. I have almost 300 different bottles of syrup at home" [Man, Rural, PP referred].
Patients expressed their wish to get more information about the disease from PPs. They were disappointed about provider's attitude and inadequate communication during consultations. We found most of the PPs, especially the non-referring ones, did not regard the counselling and conveying provisional diagnosis of TB to patients as something that was paying off for them. They rather kept patients uninformed about the provisional diagnosis of TB to avoid offending them. PPs reported that, revealing the diagnosis of TB is a sensitive issue and patients could feel insulted, if the diagnosis of TB was revealed to them up-front and they might lose patients. Instead they preferred to refer them to any higher centres. An excerpt:

"Many times even though I know this is a clear case of $T B, I$ will not reveal it to patients. You see, all these are sensitive issues. So, why should I take the risk? So, I make statements in such a sensitive way that I don't lose patients. So I persuade them go elsewhere"

[Minimally-Referring PP, Urban, General Practitioner].

\section{Discussion}

We found patients' awareness about TB was limited. Even though the symptoms were well experienced, patients did not relate them to TB. A first group of patients, self-medicated by purchasing medicines from private chemists for a long period, before seeking care. A second group sought care from government facilities and had simple itineraries. A third group, who sought care from PPs, switched concurrently and/or iteratively from public and private providers in search for relief of symptoms causing important diagnostic delays. Eventually all patients reached RNTCP and were started on treatment. PP's cross-referral practices were influenced by patient's paying capacity, familiarity with RNTCP, kickbacks from private labs and chemists, and in some cases, to get rid of TB patients. These trade-offs by PPs complicated patient's itineraries to RNTCP. Patients were disappointed about providers' attitude and inadequate communication during the consultations.

Currently RNTCP follows 'passive case finding approach'. For this strategy to work effectively, patients need to have enough knowledge to understand that they need to seek care and where in the health system this is offered. Majority of TB patients in our study had little awareness about TB before getting diagnosed with it. A study in rural India that investigated the factors associated with patient delay, attributed it to the lack of patient awareness about TB [20], a finding that is confirmed by a nation-wide cross-sectional household survey in India [21]. These findings call for an innovative health education strategy in RNTCP to increase patient's TB awareness, especially so with rural and less educated 
people. In the given scenario, community engagement becomes crucial to achieve the Government's vision of 'sweep out TB' as envisioned in its national strategic plan (NSP) of TB elimination (2017-2025). Currently systematic efforts in this direction seem to be inadequate.

About half of the patients in our study self-medicated in the initial course of illness by purchasing drug overthe counter from retail private chemists. This resonates the findings from other studies which demonstrated the importance of retail private chemists as first-contact health care providers $[22,23]$ and the missed opportunities of utilising chemist's services for early detection of TB cases [24]. Though RNTCP envisages a partnership with private chemists [25], institutionalising the processes of engaging them in RNTCP is yet to get established. Intervention research to improve the referrals from chemists to the RNTCP could contribute to the build-up of this knowledge.

Our study revealed low index of suspicion of TB among PPs. This corroborates the study findings that demonstrated sub-optimal TB management practices among PPs in India [26]. Detection of smear positive TB cases by sputum examination is a key element of DOT. Our study PPs, especially the minimally-referring and non-referring PPs ordered tests, which were not specific for TB, could have delayed the diagnosis of TB. Supporting this fact, a systematic review has shown that initially seeking care from a PP was clearly a significant risk factor for diagnostic delay [27]. Uplekar et al. in his study [28] showed that many PPs referred patients to government hospitals only late in the course of the patient's illness. As demonstrated in our study, PPs are the one who decide on the type of the diagnostic tests and medications, thus wield considerable power. PP's considerations for cross-referring cases to RNTCP were influenced by patient's paying capacity, familiarity with RNTCP, kickbacks, and in some cases, to get rid of TB patients. These trade-offs by PPs had consequently complicated patient's itineraries to RNTCP. It appears that PPs tend to see TB as source of income and not a public health responsibility. This could be corroborated with our finding which demonstrated the provision of kickbacks to PPs from private labs and chemists, a finding similar to a study from India [29]. RNTCP has made several policy changes mandating the notification of all TB patients from the private sector [30], establishing web-based TB surveillance system-NIKSHAY, ban on sero-diagnostics and amendments in $\mathrm{H} 1$ schedule to improve TB care services in private sector. But the response from the private sector to these policy changes has been poor [11]. Therefore, for effective implementation of these policies, a comprehensive, and system-oriented intervention has to be tested in the field settings, in collaboration with PPs.
We found in our study, in some instances the public sector providers were unable to diagnose TB. This is similar to the study findings from India that demonstrated the need for improvement in TB management practices in the public sector as well [31]. A recent study also found that, out of about 1.9 million TB patients who reached RNTCP in 2013, only about 1.6 million were successfully diagnosed with TB [32]. Further a recent study by Yellappa et al. has demonstrated how patients under RNTCP treatment had to cope up with stigmatisation, financial constraints and inconvenient DOT timings [16]. All these finding calls for urgent action to make RNTCP services more patients friendly within government facilities, to avoid TB patients getting deflected from RNTCP. India's NSP proposes several bold strategies to prevent the loss of TB cases in the cascade of care. But these support systems should also expand to patients managed by private sector.

Best practices for TB diagnosis and treatment are enshrined in the Indian Standards of TB Care [33]. But, studies have shown that PPs in India do not often adhere to these standards [34]. A study from Indonesia has demonstrated that exposure of PPs to the National TB program improves referral of TB cases to National TB program [35]. In our study, all the actively-referring PPs were familiar and trusted the functioning of the RNTCP. Therefore, they counseled patients when they refused to go to the RNTCP. In contrast, non-referring and minimally-referring PPs had not undergone any formal RNTCP training and they doubted the efficiency RNTCP services. Hence, it is imperative that all providers irrespective of public or private should be sensitised about RNTCP services.

The majority of patients in our study initially sought care from primary level health care providers, who failed to diagnose TB. Hence, building the capacity of primary health care providers on better use of existing tests such as sputum examination, coupled with training them on TB symptoms is essential. WHO's practical approach to lung health [36], which aims at improving the skills of primary health care workers, should be widely disseminated and implemented in India. Additionally, systematic referrals for sputum examination should be streamlined between providers and the RNTCP.

Alike other studies [37, 38], our study showed stigma surrounding TB still exists in the society and that revealing the provisional diagnosis of a stigmatised disease was perceived to be difficult by PPs [39]. This issue requires an attention, since poor communication and poor quality of information provided to patients could become an impediment to patient access to TB care [40]. Other study suggested that improved co-ordination between the PPs and the government health centres may substantially improve services for TB patients [41]. 
As an outcome of this study, an intervention has been developed to optimise the involvement of PPs and private chemists in the RNTCP. One set of interventions is targeted towards intervention private sector like training, improving communication between RNTCP staff and PPs, and other set of interventions is targeted towards general strengthening of RNTCP services [42].

The strength of this study is that it explains healthseeking trajectories from both patients' and PPs' perspective and helps to understand how TB patients navigate through the health facilities to seek care. Qualitative research method employed in this study allowed exploring patients' perceptions and experiences about TB care, corroborated with PP's TB management practices. We included a range of PPs providing TB care in different settings, which helped to identify their divergent perceptions and explore how they make decisions to refer patients to the RNTCP. This method of in-depth research to gather the narratives of TB patients and providers is useful for the programme managers to identify the weak links in the programme. However, the study findings must be interpreted with caution with regard to the specific study setting. Data was collected from such patients who accessed RNTCP. Therefore, the study does not provide any information about patients who did not reach RNTCP or died before. We have not considered patients who visited DMCs, but opted for treatment in the private sector. Documentation of patient itineraries described in the study is solely based on patient understanding and reconstruction of the events that occurred during the illness. Patients were identified from the RNTCP registers and they were initially contacted by RNTCP staff over phone to know their willingness to participate in the study. Hence, there are chances that some patients would have hesitated to share such information, which jeopardize their relationship with RNTCP staff. Patient narratives are based on reported historical events, which is vulnerable to recall bias.

\section{Conclusions}

Government of India aims for universal good quality care for all TB patients in its national strategic plan, 2017-2025. In the backdrop of bold policy changes, our study findings may help RNTCP to develop initiatives to promote early detection of TB and develop supportive pathways to patient care. Our study highlights the critical role played by private sector, including informal providers and private pharmacists catering to TB patients and the dynamics around PP's cross referral practices linked to the RNTCP. Patient's and providers' narratives from our study inform about the potential sources of delay in diagnosis and how better collaborations could be established with PPs within the realities of pluralistic health system of India. Our study has revealed the potential of private pharmacists in early TB case detection and the need for strengthening effective referral systems from private sectors to the RNTCP.

\section{Abbreviations}

DOT: Directly observed treatment; NSP: National Strategic Plan; PP; Private practitioner; RNTCP: Revised National Tuberculosis Control programme; IB: Tuberculosis

\section{Acknowledgements}

This study was funded through a PhD scholarship grant recelved by the first author from the Institute of Tropical Medicine, Antwerp, (December 2012 to November 2016). Authors are grateful to all respondents for their valuable time and for sharing their experiences. Authors thank the district TB officer and FNTCP staff of Tumkur district for faclitating the recruitment of respondents.

\section{Funding}

This work was supported by Framework agreement between the Institute of Public Health, Bangalore and the Institute of Tropical Medicine, Antwerp and the Belgium Directorate-General for Development Cocperation.

\section{Availability of data and materials}

The datasets used and/or analysed during the current study are available from the corresponding author on reasonable request.

\section{Author's contributions}

W. PL, ND and PVDS conceived and designed the study, W collected the data $\mathrm{VY}$ and $\mathrm{PL}$ analysed the data and wrote the first draft of the manuscript. PVDS, TB and ND contributed to revising the manuscript. All authors read and approved the final manuscript.

\section{Ethics approval and consent to participate}

Study received approval from the Institutional Ethics Committee of the Institute of Public Health, Bangalore and the Institutional Review Board of the Institute of Tropical Medicine, Antwerp. To avoid any breach in medical confidentiality, the PI after listing the potential TB patients, shared the list with the district TB officer, who in turn contacted field FNTCP staff to check with patients if they agreed to be interviewed. Only after patients agreed, RNTCP staff provided patients' contact details to the PL. Personal details of participants were removed to ensure confidentiality during the data transcription and analysis. Participants were informed that they could withdraw at any time from interviews and the research Written consent was obxained from all the participants by the Pl, except eight PPs who opted for verbal consent.

\section{Consent for publication}

Not applicable.

\section{Competing interests}

The authors declare that they have no competing interests.

\section{Publisher's Note}

Springer Nature remains neutral with regard to jurisdictional claims in published maps and institutional affiliations.

\section{Author details}

'Institute of Public Health, \$250, 2nd C Main, 2nd 'C Cross-, Girinagar I Phase, Bangalore, Karnataka 560 085, India. 'Institute of Tropical Medicine, Nationalestraat, 155, 2000 Antwerp, Belgium. ${ }^{3}$ Public Health Department, Faculty of Medicine, Ghent University, Ghent, Belgium.

Received: 20 April 2017 Accepted: 25 July 2017

Published online: 04 August 2017

\section{References}

1. World Health Organization. Global Tuberculosis Report. 2016. Avallable from www.who.int/tb/publications/global_report/en/. Accessed 1 Jan 2017.

2 Sachdeva KS, Satyanarayana S, Dewan PK, Nair SA, Reddy R, Kundu D, et al Source of Previous Treatment for Re-Treatment TB Cases Registered under the National TB Control Programme, India, 2010. PLOS ONE. 201 1,6(7):22061. 
3. Satyanarayana S, Nair SA. Chacha SS, Shivashankar R, Sharma G, Yadav S, et al. From where are tuberculosis patients accessing treatment in India? Results from a cross-sectional community based survey of 30 districts. PLoS One. $20116(9)$ e 24160

4. Satyanarayana S, Subbaraman R, Shete P, et al. Quality of tuberculosis care in India a systematic review, int J Tuberc Lung Dis. 2015;1977),751-63. do:10.5588/itid.15.0186.

5. Udwadia ZF, Pinto LM. Uplekar MW. Tuberculosis management by private practitioners in Mumbai, India: has anything changed in two Decades? PLoS One, 2010:5(8):1-5.

6. Central TB Division, Directorate General of Health Services, Ministry of Health with Family Welfare. National Guideline for Partnership. Government of India. 2015. Available from http//tbcindianicin/showfile.php?lid=3143. Accessed 12 Dec 2016.

7. Yelappa V, Battaglioli T, Gurum SK, Narayanan D, Van Der Stuyft P. Private practitioners contributions to the revised National Tuberculosis Control Programme in a south Indian district. Int J Tuberc Lung Dis. 2016:20(5),659-65.

8. Lal SS, Sahu S, Wares F, Lönnroth K, Chauhan LS, Uplekar M. Intensified scale-up of public-private mix a systems approach to tuberculosis care and control in India. Intern J Tuberc lung Dis. 2011;15:97-104.

9. Dewan PK, Lal SS, Lonnroth K Wares F, Uplekar M, Sahu S, et al. Improving tuberculosis control through public-private collaboration in India: literature review. BMU. 20063332574-8

10. Rajeswari R, Chandrasekaran V, Suhadev M, Sivasubramaniam S, Sudha G, Penu G. Factors associated with patient and health system delass in the diagnosis of tuberculosis in South India. Int J Tuberc Lung Dis. 2002,669)/789-95.

11. Philip S, Isaakidis P, Sagili KD, Meharunnisa A Mrithyuniayan S, Kumar AMV. They know, they agree, but they don't do"- the paradox of tuberculosis case notification by private practitioners in Alappuzha District, Kerala, India. PLoS One. 2015:10(4) re0123286.

12. Suganthi P, Chadha VK, Ahmed J, Umadevi G, Kumar P, Srivastava R et al, Health seeking and knowledge about tuberculosis among persons with pulmonary symptoms and tuberculosis cases in Bangalore slums. Int Tuberc Lung Dis. 2008:12(11):1268-73.

13. Charkes N, Thomas B, Watson B, Raja Sakthivel M, Chandrasekeran V, Wares F. Care seeking behavior of chest symptomatics: a community based study done in South India after the implementation of the RNTCP. PLOS One. $20105(9) \cdot 1-6$.

14. Sreeramareddy $C T$, Qin ZZ, Satyanarayana S, Subbaraman R, Pai M. Delays in diagnosis and treatment of pulmonary tuberculosis in India: a systematic review. Int J Tuberc Lung Dis. 2014;18:25S-66.

15. Prasad R, Nautilyal RG, Mukherii PK, Jain A, Singh K, Ahuja AC. Diagnostic evaluation of pulmonary tuberculosis: what do doctors of modem medicine do in India? Int J TB Lung dieases. 2003;7:52-7.

16. Yellappa V, Lefèvre P, Battaglioli T, Narayanan D. Van Der Stuyt P. Coping with tuberculosis and directly coserved treatment: a qualitative study among patients from South India. BMC Health Serv Res. 2016;16:283. do: $10.1186 / s 12913-016-1545-9$.

17. Burnard $P$, GIII $P$, Stewart $K$, Treasure E, Chadwick $B$. Analysing and presenting qualitative data. Br Dent J. 2008,204(8),429-32.

18. Boyatzis RE. Transforming qualitative information, thematic analysis and code development. Thousand Oaks CA: Sage: 1998

19. Patton MQ. Qualitative research and evaluation methods. 3rd ed. Sage: Thousand Oaks, CA: 2002

20. Nimbarte SB, Wagh V, Selokar D. Health seeking behaviour among pulmonary tuberculosis patients in rural part of central India. Int J Biol Med Res. 2011:2(1):394-7.

21. Sreeramaredoy CT, Harsha Kumar HN, Arokiasamy J. Prevalence of selfreported tuberculosis, knowledge about tuberculosis transmission and its determinants among adults in India: results from a nation-wide crosssectional household survey. BMC Infect Dis. 2013;13(1):16.

22. Mesfin MM, Newell JN, Walley JD, Gessessew A, Madeley R. Delayed consultation among pulmonary tuberculosis patients; a cross sectional study of 10 DOTS districts of Ethiopia. BMC Public Health. 2009,9(1):53.

23. Satyanarayana S, Kwan A, Daniels B, Subbaraman R, Mcdowell A. Bergkvist S, et al. Use of standardised patients to assess antibiotic dispensing for tuberculosis by pharmacies in urban India: a cross-sectional study. Lancet Infect Dis. 2016;3099(16):1-8.

24. Rajeswari R, Balasubramanian R, Bose MSC. Sekar L. Rahman F. Private pharmacies in tuberculosis control - a neglected link. Int J Tuberc Lung Dis. $2002,6(2): 171-3$.
25. Training module for community phamacists. Central TB Division Directorate General of Health Services, Ministry of Health with Family Welfare. 2013. Avallable from http//tbcindlanicin/showfle.php?lid=3154. Accessed 14 Feb 2017.

26. Satyanarayana S, Subbaraman R Shete P, Gore G, Das d, Cattamanchi A et al. Quality of tuberculosis care in India: a systematic review. Int J Tuberc Lung Dis. 2015;19.751-63.

27. Sreeramareddy $\subset$. Zhen Qin Z, Satyanarayana S, Subbaraman R. Pai $M$ Rahman A, et al. Delays in diagnosis and treatment of pulmonary tuberculosis in India: a ssstematic review, Int J Tuberc Lung Dis. 2014,18(3):255266

28. Uplekar M. Juvekar S, Morankar S. Rangan S. Nunn P. Tuberculosis patients and practitioners in private clinics in India. Intern J Tuberc lung Dis. 1998:2(4):324-9.

29. VYellappa, Narayanan Devadasan NR. Evaluation of Results based financing strategies. 2014. Available from http///phindla.org/wp-content/uploads/ 2015/10/Evaluation-of-Results-Rased-Financing-Strategies-for-Tuberculosiscare-and-Control-in-India pdf.

30. Direchiealth and Family Welfare, Government of India central TD. Guidance for TB Notification in India. 2012

31. Mishra G. Mulani J. Tuberculosis prescription practices in private and public sector in India. Natl J Integr Res Med. 2013;4(2):71-8.

32. Subbaraman R, Nathavitharana RR, Satyanarayana S, Pai M, Thomas BE, Chadha WK et al. The tuberculosis Cascade of Care in India's public sector a systematic review and meta-analysis. PLoS Med. 2016;13(10)e 1002149.

33. World Health organisation Standards for TB care in India. 2014. Available from http//www.tbcindia.nicin/showflephp?lid=3061. Accessed 4 Feb 2017.

34. Achanta S, Jaju d, Kumar AMN, Nagaraja SB, Shamrao SRM, Bandi SK et al, Tuberculosis management practices by private practitioners in Andhra Pradesh India. PLoS One. 20138(8) re71119.

35. Artawan Eka Putra IWG Utami NWA, Suarjana I, IMK D, CID A, Putra L, et al. Factors associated to referral of tuberculosis suspects by private practitioners to community health centres in Bali Province, Indonesia. BMC Health Serv Res. 2013:13(1):445

36. World Health Organization. Practical Approach to Lung Health-Manual on Initiating PAL. Implementation, 2008, Available from: https $/ /$ www.ncbinlm. nih.gov/books/NBK310624/. Accessed 17 Apr 2017

37. Somma D, Thomas BE, Karim F, et al Gender and socio-cultural determinants of TB-related stigma in Bangladesh, India, Malawi and Colombia. Int J Tuberc Lung Dis. 2008:12(7):856-66.

38. Jaggarajamma, K, Ramachandin R, N Charles, et al. Psycho-social dysfunction; perceived and enacted stigma among tuberculosis patients registered under revised national tuberculosis control programme Indian J Tuberc. 2008:31(44).

39. Miller R, Parkhurst JO, Peckham S, Singh R8. A qualitative study of HIV testing and referral practices of private hospital doctors treating patients with TB in Chennai, India. Int J Health Plann Manage. 2012:27(2):180-91. doi:10.1002/hpm.2105.

40. Khan A. Walley J, Newell J, Imdad N. Tuberculosis in Pakistan: socio-cultural constraints and opportunities in treatment. Soc Sci Med. 2000,50(2):247-54

41. Hane F, Thiam S, Fall AS, Vidal L, Diop AH, Ndir M, et al. Identifying barriers to effective tuberculosis control in Senegal: an anthropological approach Int J Tuberc Lung Dis. 2007:11(5)539-43.

42. http://www.who.int/tdr/news/2015/impact-grant/en/.

Submit your next manuscript to BioMed Central and we will help you at every step:

- We accept pre-submission inquiries

- Our selector tool helps you to find the most relevant journal

- We provide round the clock customer support

- Convenient online submission

- Thorough peer review

- Inclusion in PubMed and all major indexing services

- Maximum visibility for your research

Submit your manuscript at www.biomedcentral.com/submit 


\section{CHAPTER 4}

This chapter is based on the paper titled, 'Involving private practitioners in the Indian tuberculosis programme: A randomised trial'. Here, the implementation of intervention and the results from randomised control trial is described. This chapter also discusses the health system challenges in building the partnership between private and public counterparts. 


\title{
Involving private practitioners in the Indian tuberculosis programme: a randomised trial
}

\author{
Vijayashree Yellappa ${ }^{1,2, *}$, Tullia Battaglioli ${ }^{1 * *}$, Sanath Kumar Gurum ${ }^{3}$, Devadasan Narayanan ${ }^{2}$ and \\ Patrick Van der Stuyft ${ }^{1,4}$
}

1 Department of Public Health, Institute of Tropical Medicine, Antwerp, Belgitam

2 Institute of Public Health, Bangalore, India

3 Department of Health and Family Welfare, Karnataka Government, Bangalore, India

4 Department of Public Health, Faculty of Medicine, Ghent University, Gbent, Belgium

\begin{abstract}
OBJECTIVES To assess a multicomponent intervention to improve private practitioners (PPs) involvement in referral of presumptive pulmonary TB (PTB) cases to the Revised National TB Control Programme (RNTCP) for sputum examination.

METHODS Randomised controlled trial. We randomly allocated all 189 eligible PPs in Tumkur city, South India, to intervention or control arm. The intervention, implemented between December 2014 and January 2016, included two sets of activities, one targeted at health system strengthening (building RNTCP staff capacity to collaborate with PPs, provision of feedback on referrals through SMS) and one targeted at intervention PPs (training in RNTCP, provision of referral pads and education materials and monthly visits to PPs by RNTCP staff). Crude and adjusted referral and PTB case-finding rate ratios were calculated with negative binomial regression.

RESULTS PPs referred 836 individuals ( 548 from intervention and 169 from control arm PPs) of whom 176 were diagnosed with bacteriologically confirmed PTB. The proportion ( $95 \%$ confidence interval) of referring PPs $[0.59(0.49,0.68)$ vs. $0.42(0.32,0.52)$ in the intervention and control arm, respectively], mean referral rate per PP-year $[(5.7(3.8,8.7)$ vs. $1.8(1.2,2.8)]$ and smear-positive PTB case-finding rate per PP-year $[(1.5(0.9,2.2)$ vs. $0.6(0.3,0.9)]$ were significantly higher in the intervention than the control arm. Stratifying by qualification, a statistically significant difference in the above indicators remained only among GPs and internists. Overall, surgeons, paediatricians and gynaecologists referred few patients. PP referrals contributed to $20 \%$ of the sputum smear positive PTB cases detected by RNTCP in Tumkur city (14\% were from intervention arm PPs).

CONCLUSions We demonstrated the effectiveness of a health system-oriented intervention to improve PP's referrals of presumptive PTB cases to RNTCP.
\end{abstract}

keywords PPM, private practitioner, referral, tuberculosis, South India, randomised controlled trial, RNTCP

\section{Introduction}

In 2015, Asia accounted for $61 \%$ of the estimated 10.4 million new tuberculosis (TB) cases globally, with India leading the count [1]. A gap of 4.3 million was estimated between notified and estimated cases, due to underreporting and underdiagnosis. India was among the 10 countries accounting for $77 \%$ of the gap [1]. The Indian Revised National TB Control Programme (RNTCP), launched in 1997, is implemented mainly through public sector health facilities. However, the country has a large

"Co-first authors. unregulated private sector and the first point of contact for health care for many patients are private providers (PPs) $[2$, $3]$, where care is often substandard and inconsistent $[4,5]$. Indiscriminate use of non-recommended TB diagnosis workup leads to considerable diagnostic and treatment delays $[6,7]$. Patients often consult multiple providers before being correctly diagnosed, incurring high costs [8]. All this has catastrophic effects on TB control efforts.

Since 2006, India has been implementing the publicprivate mix strategy (PPM), recommended by World Health Organization (WHO), to engage PPs in TB care and control, but achievements remain far from complete $[9,10]$. Despite the promise shown by PPM models [11], 
their expansion in most settings is relatively limited. Furthermore, the evidence base of PPM effectiveness is considered rather weak [12] and several unknowns remain regarding initiatives to effectively and sustainably engage PPs [13]. In India, since 2012 it is mandatory for PPs to notify TB cases diagnosed outside RNTCP [14] but notifications remain low [15].

Against this background, we developed and tested a multicomponent intervention to increase the involvement of allopathic PPs in the referral of presumptive pulmonary TB (PTB) cases to RNTCP and TB case notification in a South Indian district.

\section{Materials and Methods}

\section{Setting}

The study took place in Tumkur city (population 384 016), the headquarters of Tumkur district (population 2.7 million), Karnataka state, India. In the district, free quality sputum microscopy (Ziehl-Neelsen staining) is provided by 28 Designated Microscopic Centres (DMCs), part of the RNTCP laboratory network, each covering a population of around 100000 . Since April 2015, the District TB Centre has a GeneXpert machine. Patients either directly access government health facilities or are referred by PPs.

\section{Design}

We conducted a two-arm, randomised controlled trial: PPs were randomly allocated, blocking by speciality, to the intervention and control arms. The study ran from September 2014 to January 2016.

\section{Participants}

PPs were considered for inclusion if they were for-profit, allopathic, 'formal' (with an official medical degree), qualified as general practitioner (GP) or internist, surgeon, paediatrician or gynaecologist (deemed more likely to be consulted by chest symptomatics than other qualifications) and practising in a private solo clinic (providing outpatient care) and/or nursing home (health structure providing inpatient care, with nursing staff and a medical doctor in charge around the clock) in Tumkur city. PPs practising less than one day per week or employed by the government were excluded.

Before starting, a census of for-profit allopathic formal PPs practising in the city was carried out (April-September 2014), using GPS technology, by three trained RNTCP TB-Health Visitors and one research staff. A total of 316 PPs were identified. Information on qualification, sex, age, years of experience, involvement with RNTCP as directly observed treatment (DOT) provider, participation to an RNTCP sensitisation workshop conducted in March 2014 in the district (prior and unrelated to the study), affiliation to a medical college and average number of outpatient consultations/month were recorded.

\section{Intervention}

The design of the intervention was preceded by a phase of formative research. A quantitative study [10] explored the extent and nature of PPs collaboration in RNTCP in the district, and qualitative research $[16,17]$ involving TB patients, RNTCP staff and all types of PPs explored barriers to effective PPM and potential and possible ways to engage PPs. Based on the findings of the formative phase and together with the district TB office, a package of intervention activities was designed: one set of activities, started in September 2014, targeted general strengthening of RNTCP services; another set, started in October 2014, targeted exclusively the PPs in the intervention arm (Box 1].

No financial incentives were given to participating PPs. Activities were implemented by RNTCP staff, under supervision of the DTO, with technical assistance from the other investigators.

\section{Outcomes}

The main considered outcomes were proportion of PPs referring at least one presumptive PTB case to RNTCP ('referring PPs') and rate of referral of presumptive PTB cases per PP-year. Secondary outcomes were proportion of PPs notifying at least one TB case and rate of detection of bacteriologically confirmed PTB cases per PP-year. Power calculation indicated that a sample of 100 PPs per study arm would achieve a power of $84 \%$ to detect a six times higher referral rate in the intervention compared to the control arm, expecting a referral rate of two referrals per PP-year in the control arm [10], at a significance level of 0.05 , using a one-sided test. The same sample size would achieve $99 \%$ power to detect a two times difference in the proportion of referring PPs between the arms, expecting a proportion of 0.3 in the control arm [10].

\section{Data collection and analysis}

Data on referrals and notifications from December 2014 to January 2016 (14 months) were extracted from the district's DMC and TB treatment registers and from referral and notification forms. Information on presumptive PTB cases examined at the DMCs, source of referral, 
V. Yellappa et al. Private practitioners in the Indian TB program

Box I Summary of the intervention package implemented in Tumkur city, September 2014-January 2016

Activities targeted at general strengthening of RNTCP services:

(i) RNTCP staff refresher training on RNTCP guidelines and PPM schemes, sensitisation on the importance of collaboration and communication with PPs, detailed instructions on intervention components, procedures and tools;

(ii) Strengthening of the whole information system and in particular documentation of the source of referral in the district's DMCs laboratory registers (training of laboratory technicians, supervision by senior TB laboratory supervisor);

(iii) Streamlining the reporting of PPM activities, with periodic monitoring of PPs' referrals and staff meetings to discuss PPM activities;

(iv) Improved DMCs accessibility for patients, with extended opening hours, placement of signboards with directions to the DMCs in the health facilities, sensitisation of their help desk and registration counter staff to help patients reach the DMC;

(v) Counselling of patients by DMCs laboratory technicians on going back to the treating physician with the sputum examination results and orientation about RNTCP treatment options and procedures; and

(vi) Provision of phone directory of study PPs to RNTCP staff.

Activities targeted exclusively at PPs included in the intervention arm

(i) Half-day (2 pm to $6 \mathrm{pm}$ ) training in standards of TB care, RNTCP guidelines, PPM schemes, how to become a DOT provider, notification of TB cases, intervention components, procedures and tools; PPs were invited by the DTO; the meeting was held at Indian Medical Association in Tumkur city; Karnataka Medical Association Continuing Medical Education credit points from Karnataka Medical Council were awarded to participating PPs; and the training was led by RNTCP staff;

(ii) Visits by DTO to clinics of intervention PPs who did not participate in the training to secure participation, communicate essential content of what was covered during the half-day training and provide study tools;

(iii) Strengthening communication between RNTCP and PPs through:

(a) Sending short message service (SMS) with results of sputum examination and information on whether referred patients started RNTCP treatment;

(b) Monthly visits to the PPs' clinics by TB-Health Visitors, to provide feedback on referred patients and retrieve and replenish referral slips and notification forms; and

(c) Provision of phone directory of key RNTCP staff;

(iv) Provision of TB information-education-communication material for patients and maps with directions of DMCs.

sputum examination results, HIV testing and TB treatment outcome (for those opting for RNTCP treatment) were entered in a Microsoft Excel (2010) sheet monthly by a trained data entry operator. A presumptive PTB case referred by a PP was defined as a patient undergoing a diagnostic sputum examination, whose source of referral was a PPs' private practice, as recorded in the DMC registers.

The difference in proportion of referring PPs and median number of referrals per PP per year between the study arms, with $95 \%$ confidence interval $(95 \% \mathrm{CI})$ (bootstrapped for medians), were calculated. Crude referral and PTB case-finding rates and crude and adjusted rate ratios (RR) with $95 \%$ CI were calculated with negative binomial regression. Analyses were performed using IBM SPSS Statistics Version 23.0.

\section{Ethics}

The institutional ethics committee of the Institute of Public Health, Bangalore, and the institutional review board of Institute of Tropical Medicine, Antwerp, Belgium, approved the study. The District TB Officer (DTO) invited the 94 intervention arm PPs to participate in the half-day training, prior to the start of the intervention, where their written consent to participate in the study was obtained. Consent from the 63 PPs who did not attend the initial training was obtained during individual visits to their clinics by the DTO to secure participation, communicate essential content of what was covered during the half-day training and provide study tools.

\section{Trial registration}

The study was registered in the Clinical Trial RegistryIndia (CTRI) under registration number CTRI/2017/09/ 009672 .

\section{Results}

A total of 189 PPs were enrolled (Figure 1); their median age (interquartile range) was 46 years (39-59); they had a median of $18(8-28)$ years of experience; and 
Figure I Flow chart showing participant selection and randomisation to intervention and control arm, October 2014, Tumkur city, Karnataka, India.
316 formal for profit allopathic private practitioners practicing in Tumkur city

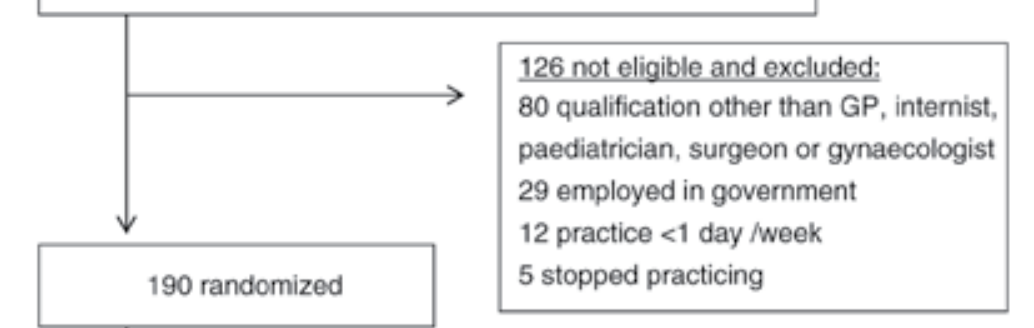

1 general practitioner randomized to intervention excluded after randomization (reason: turned out to work in government)

\begin{tabular}{|c|c|c|c|}
\hline \multicolumn{2}{|c|}{94 in intervention arm } & \multicolumn{2}{|c|}{95 in control arm } \\
\hline Qualification & $\mathrm{Nr}$ & Qualification & $\mathrm{Nr}$ \\
\hline General practitioner & 20 & General practitioner & 21 \\
\hline Internist & 21 & Internist & 22 \\
\hline Paediatrician & 19 & Paediatrician & 18 \\
\hline Surgeon & 16 & Surgeon & 16 \\
\hline Gynaecologist & 18 & Gynaecologist & 18 \\
\hline
\end{tabular}

Table I Number and proportion of private practitioners making at least one referral to RNTCP, by study group and by qualification, during the intervention period, December 2014-January 2016, Tumkur district, Karnataka, India

\begin{tabular}{|c|c|c|c|c|c|}
\hline \multirow[b]{2}{*}{ Qualification } & \multicolumn{2}{|c|}{ Intervention } & \multicolumn{2}{|l|}{ Control } & \multirow{2}{*}{$\begin{array}{l}\text { Difference in \% referring } \\
(95 \% \mathrm{CI})(P \text { value })\end{array}$} \\
\hline & Total No. & Referring, No. (\%) & Total No. & Referring, No. $(\%)$ & \\
\hline All & 94 & $55(59)$ & 95 & $40(42)$ & $17.0(3.0,31.0)(0.02)$ \\
\hline General practitioner & 20 & $19(95)$ & 21 & $13(62)$ & $33.0(10.1,55.9)(0.02)^{\text {क }}$ \\
\hline Internist & 21 & $15(71)$ & 22 & $12(55)$ & $16.0(-12.4,44.4)(0.25)$ \\
\hline Paediatrician & 19 & $10(53)$ & 18 & $6(33)$ & $20.0(-11.2,51.2)(0.23)$ \\
\hline Surgeon & 16 & $9(56)$ & 16 & $6(38)$ & $18.0(-16.0,52.0)(0.28)$ \\
\hline Gynaecologist & 18 & $2(11)$ & 18 & $3(17)$ & $-6.0(-28.6,16.6)(1.00)^{2}$ \\
\hline
\end{tabular}

CI, confidence interval.

"Fisher exact test.

$77 \%$ were males, $29 \%$ had participated in a previous RNTCP workshop, with no difference between the study arms. The proportion of DOT providers was higher in the intervention than in the control arm $(20 \%$ vs. $11 \%)$.

During the study period, 836 presumptive PTB cases were referred by study PPs ( 830 for sputum smear microscopy, six for GeneXpert); $62 \%$ of these were male and $95 \%$ over 15 years old. Eleven (3\%) of 323 individuals tested for HIV were positive.

The proportion of referring PPs was significantly higher in the intervention than in the control arm; stratifying by qualification, a statistically significant difference remained only among GPs (Table 1). Gynaecologists in both arms 
showed the lowest figures. The median number of referrals per PP per year was significantly higher in the intervention than in the control arm (Table 2). Stratified analysis showed a significant difference among GPs and internists only, who, in both arms, had a higher median number of referrals than other specialists. The referral rate per PP-year was nearly two times higher in the intervention than in the control arm; after stratification by qualification, the effect of the intervention remained only among GPs and internists (Table 3).

During the study period, 176 individuals referred by PPs were diagnosed with bacteriologically confirmed PTB. The PTB case-finding rate per PP-year was 1.7 times higher in the intervention than in the control arm; after stratification, the intervention showed an effect only among GPs and internists (Table 4). No cases were found among referrals by gynaecologists. The proportion $(95 \% \mathrm{CI})$ of bacteriologically confirmed PTB cases among referrals was higher in the control $(26 \%, 20-32)$ than in the intervention arm $(20 \%, 16-23)$, but this difference was not significant. Among the PTB cases, 129 $(73 \%)$ started RNTCP TB treatment: $102(79 \%)$ were cured, $6(5 \%)$ completed treatment, $8(6 \%)$ died, 7 (5\%) were lost to follow-up, $4(3 \%)$ failed, $1(1 \%)$ shifted to MDR-TB treatment and $1(1 \%)$ was not evaluated.

Study PPs' referrals contributed to $10 \%$ of the total yearly number of examinations at Tumkur city's DMCs and contributed to $20 \%$ of the smear-positive PTB cases detected by DMCs (Figure 2).

Apart from this, there were 50 notifications from study PPs, of which $34(68 \%)$ were PTB cases (which, on a

Table 2 Total number of referrals per group per year, median number of referrals per private practitioner per year and difference in medians by study group and by qualification, during the intervention period, December 2014-January 2016, Tumkur district, Karnataka, India

\begin{tabular}{|c|c|c|c|c|c|}
\hline \multirow[b]{2}{*}{ Qualification } & \multicolumn{2}{|l|}{ Intervention } & \multicolumn{2}{|l|}{ Control } & \multirow[b]{2}{*}{$\begin{array}{l}\text { Difference in } \\
\text { medians }(95 \% \mathrm{Cl}) \\
(P \text { value })\end{array}$} \\
\hline & $\begin{array}{l}\text { Total No. referrals } \\
\text { per group per year }\end{array}$ & $\begin{array}{l}\text { Median No. referrals } \\
\text { per PP per year } \\
\text { (Q1, Q3) }\end{array}$ & $\begin{array}{l}\text { Total No. } \\
\text { referrals } \\
\text { per group } \\
\text { per year }\end{array}$ & $\begin{array}{l}\text { Median No. } \\
\text { referrals } \\
\text { per PP per } \\
\text { year (Q1, Q3) }\end{array}$ & \\
\hline All & 548 & $0.9(0.0,5.1)$ & 169 & $0.0(0.0,1.7)$ & $0.9(0.0,1.7)(0.003)$ \\
\hline General practitioner & 130 & $5.1(2.6,8.1)$ & 18 & $0.9(0.0,1.3)$ & $4.2(1.7,5.6)(<0.001)$ \\
\hline Internist & 277 & $7.7(0.0,21.0)$ & 76 & $1.3(0.0,5.4)$ & $6.4(-0.9,17.2)(0.05)$ \\
\hline Paediatrician & 17 & $0.9(0.0,1.7)$ & 11 & $0.0(0.0,1.1)$ & $0.9(-0.5,1.7)(0.36)^{6}$ \\
\hline Surgeon & 121 & $0.9(0.0,3.4)$ & 53 & $0.0(0.0,1.5)$ & $0.9(-0.9,0.9)(0.40)^{*}$ \\
\hline Gynaecologist & 3 & $0.0(0.0,0.0)$ & 10 & $0.0(0.0,0.0)$ & $0.0(0.0,0.0)(0.72)^{5}$ \\
\hline
\end{tabular}

$\mathrm{PP}$, private practitioner; $\mathrm{Q}$, quartile; $\mathrm{Cl}$, confidence interval.

'Exact significance.

Table 3 Crude referral rate per private practitioner-year and crude and adjusted referral rate ratios by study group and by qualification, during the intervention period, December 2014-January 2016, Tumkur district, Karnataka, India

\begin{tabular}{|c|c|c|c|c|}
\hline \multirow[b]{2}{*}{ Qualification } & \multicolumn{2}{|c|}{ Crude referral rate per PP-year $(95 \% \mathrm{CI})$} & \multirow{2}{*}{$\begin{array}{l}\text { Crude referral rate } \\
\text { ratio }(95 \% \mathrm{Cl})\end{array}$} & \multirow{2}{*}{$\begin{array}{l}\text { Adjusted referral } \\
\text { rate ratio }(95 \% \mathrm{CI})^{*}\end{array}$} \\
\hline & Intervention & Control & & \\
\hline All & $5.7(3.8,8.7)$ & $1.8(1.2,2.8)$ & $3.2(1.8,5.8)$ & $1.9(1.2,3.0) \dagger$ \\
\hline General practitioner & $6.7(4.6,9.7)$ & $1.0(0.6,1.7)$ & $6.8(3.6,12.9)$ & $5.4(2.9,10.1)$ \\
\hline Internist & $13.3(6.6,25.5)$ & $3.5(1.4,7.4)$ & $3.8(1.4,10.3)$ & $2.2(1.0,4.8)$ \\
\hline Paediatrician & $0.9(0.4,2.0)$ & $0.6(0.2,1.5)$ & $1.4(0.5,3.8)$ & $1.6(0.7,3.7)$ \\
\hline Surgeon & $7.6(2.4,24.0)$ & $3.3(0.9,13.4)$ & $2.3(0.4,13.5)$ & $2.3(0.6,9.1)$ \\
\hline Gynaecologist & $0.2(0.0,0.7)$ & $0.6(0.2,1.9)$ & $0.2(0.0,1.7)$ & $0.1(0.0,1.6)$ \\
\hline
\end{tabular}

$\mathrm{PP}$, private practitioner; $\mathrm{CI}=$ confidence interval.

"Adjusted for having attended latest RNTCP sensitisation workshop, being DOT provider, average number of outpatient consultations' month.

$†$ Also adjusted for qualification. 
yearly basis, would constitute roughly $3 \%$ of all PTB cases notified in Tumkur city's DMCs); all but one notification was from 12 intervention arm PPs, where two internists were responsible for $25(50 \%)$.

\section{Discussion}

A multicomponent intervention integrated into the RNTCP was effective at improving the involvement of private GPs and internists in referral of presumptive PTB cases for sputum examination to RNTCP. Overall, surgeons, paediatricians and gynaecologists referred few patients. Only a minority of PPs, mostly in the intervention arm, notified TB cases.

A strength of this study is the randomised controlled trial design. Despite the abundance of literature describing PPM programmes, robust empirical evidence of their effectiveness on programme outcomes is limited and encompassing mostly cross-sectional or longitudinal studies, many without controls and apparently none with a randomised design [18]. The intervention was designed from evidence gathered in the field, refined in consultation with stakeholders and was well integrated into the NTP, assuring sustainability. All eligible PPs were randomised, and all PPs allocated to the intervention group accepted to participate in the study, making it well representative.

A first study limitation, some would argue, is that, since all the intervention activities were implemented simultaneously and general RNTCP strengthening activities went hand in hand with activities targeted at intervention PPs, it was not possible to determine which particular activity was essential to obtain the observed effect. However, this is characteristic of (and even essential for) system-oriented interventions. Another limitation is that we focused on sputum examinations and did not systematically record referrals to RNTCP for chest X-ray or Mantoux test, possibly slightly underestimating the overall volume of PPs' referrals. Further, only formal forprofit allopathic PPs were included but, as in the rest of the country, the district's private sector is more diverse, including formal non-allopathic and informal practitioners who potentially may also be consulted by chest symptomatics. However, our earlier research [10] showed that in this urban setting, allopathic PPs constituted the vast majority and were the ones showing most potential for referral of presumptive TB cases, which justified their prioritisation. Also, some underreporting of PP referrals by laboratory technicians cannot be excluded (but there is no reason to assume that it would be differential).

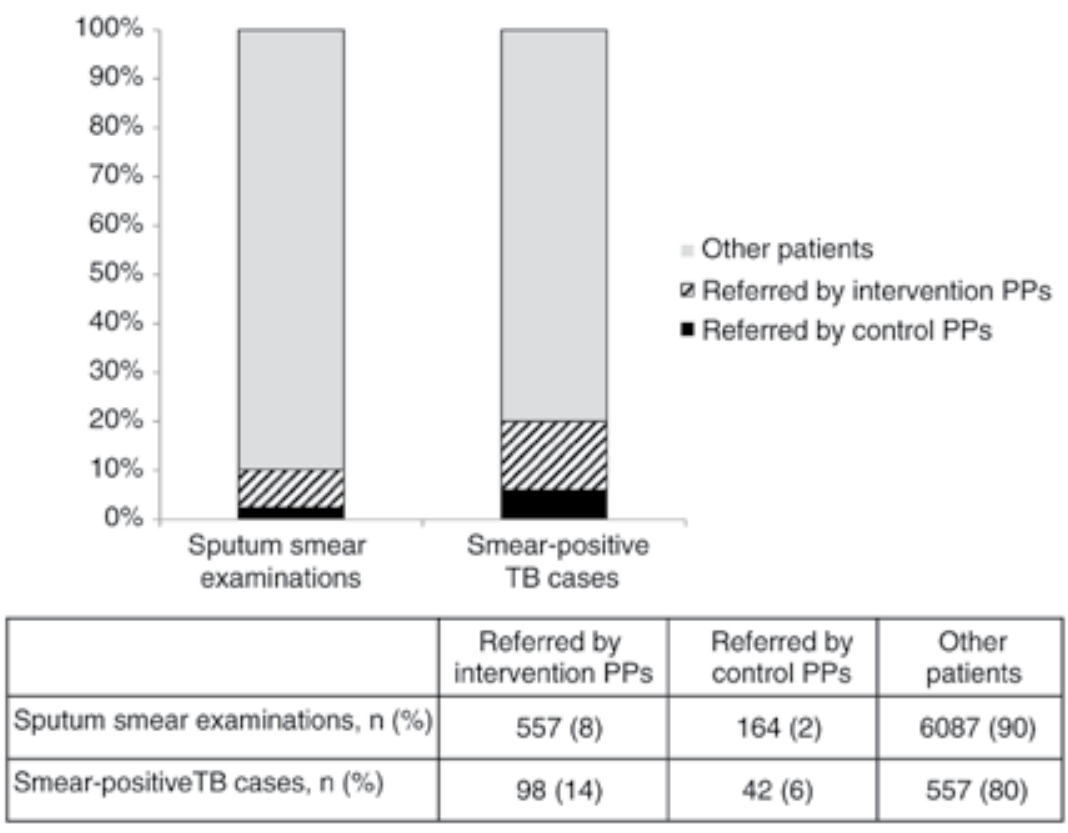

Figure 2 Contribution to study private practitioners' referrals to yearly sputum smear examinations and to smear-positive TB casefinding in Tumkur city's Designated Microscopy Centres, during the intervention period, December 2014-January 2016, Tumkur, Karnataka, India. (PP = private practitioner; $\mathrm{TB}$ - tuberculosis). 
V. Yellappa et al. Private practitioners in the Indian TB program

Table 4 Total number of bacteriologically confirmed pulmonary TB cases among referrals to RNTCP per group per year, crude TB case-finding rate per private practitioner-year and crude and adjusted TB case-finding rate ratios, by study arm and by qualification, during the intervention period, December 2014-January 2016, Tumkur district, Karnataka, India

\begin{tabular}{|c|c|c|c|c|c|c|}
\hline \multirow[b]{2}{*}{ Qualification* } & \multicolumn{2}{|l|}{ Intervention } & \multicolumn{2}{|l|}{ Control } & \multirow[b]{2}{*}{$\begin{array}{l}\text { Crude case-finding } \\
\text { rate ratio }(95 \% \mathrm{CI})\end{array}$} & \multirow[b]{2}{*}{$\begin{array}{l}\text { Adjusted } \\
\text { case-finding } \\
\text { rate ratio } \\
(95 \% \mathrm{CI}) \dagger\end{array}$} \\
\hline & $\begin{array}{l}\text { No. of TB cases } \\
\text { per group } \\
\text { per year }\end{array}$ & $\begin{array}{l}\text { Crude TB } \\
\text { case-finding } \\
\text { rate per PP-year } \\
(95 \% \mathrm{CI})\end{array}$ & $\begin{array}{l}\text { No. of TB cases } \\
\text { per group } \\
\text { per year }\end{array}$ & $\begin{array}{l}\text { Crude TB } \\
\text { case-finding rate } \\
\text { per PP-year } \\
(95 \% \mathrm{CI})\end{array}$ & & \\
\hline All & 107 & $1.5(0.9,2.2)$ & 44 & $0.6(0.3,0.9)$ & $2.4(1.3,4.6)$ & $1.7(1.0,2.8) \ddagger$ \\
\hline General practitioner & 25 & $1.3(0.9,2.0)$ & 7 & $0.3(0.2,0.8)$ & $3.4(1.4,8.1)$ & $2.8(1.1,7.1)$ \\
\hline Internist & 72 & $3.5(1.8,6.9)$ & 20 & $0.9(0.5,1.8)$ & $3.8(1.5,9.8)$ & $2.5(1.2,5.3)$ \\
\hline Paediatrician & 3 & $0.2(0.1,0.5)$ & 3 & $0.2(0.1,0.6)$ & $0.9(0.2,3.8)$ & $1.0(0.2,4.9)$ \\
\hline Surgeon & 7 & $0.4(0.2,1.5)$ & 14 & $0.9(0.2,3.6)$ & $0.5(0.1,3.4)$ & $1.0(0.1,13.0)$ \\
\hline
\end{tabular}

$\mathrm{TB}$, tuberculosis; $\mathrm{PP}$, private practitioner; $\mathrm{CI}$, confidence interval.

- Since no cases were found among referrals by gynaecologist in both study groups, they were excluded.

†Adjusted for having attended latest RNTCP sensitisation workshop, being DOT provider, average number of outpatient consultations' month.

‡Also adjusted for qualification.

Moreover, the number of patients reaching the DMCs could be lower than the number actually referred by PPs, but we could not assess this because of inconsistent use of referral slips by PPs.

The implementation of the intervention was not without challenges. Building and maintaining reciprocal trust between PPs and RNTCP was a time- and resource-intensive endeavour, demanding strong and continuous commitment from the public sector. Also, over half of the intervention PPs did not participate in the initial training, which required ad hoc visits to their clinics by the DTO, to secure their participation and training. Repeat visits by one of the first authors (V.Y.) further served this purpose. Furthermore, during implementation, it appeared that day-to-day care of patients admitted by PPs in private nursing homes was often provided by duty doctors and head nurses, who were also often responsible for the actual execution of PPs' referrals to RNTCP so and an additional training for them had to be organised. Improving DMC accessibility by patients was only partially achieved: DMCs did expand their opening hours but signposts to the DMCs, although available, were not placed in all health facilities and maps were not consistently distributed by PPs to patients.

For a successful PPM, the first and foremost step is knowing the private sector landscape. Complete and updated information about PPs practising in Tumkur city was unavailable, and the PP census was time- and resource-intensive. However, it allowed us to know the absolute number of PPs and the use of GPS technology informed on their geographical distribution. Considering how dynamic is the private sector, periodic mapping updates are necessary. Synergy with other health programmes benefitting from knowledge of the private sector landscape could facilitate this exercise.

Most of the past reported successes of PPM have come from engagement with big hospitals and medical colleges [19-21], which, in contrast to individual PPs, are deemed likely to yield a larger number of cases. We demonstrated that solo PPs can contribute to RNTCP. Some PPs appeared worth prioritising compared to others: GPs and internists had a higher volume of referrals, and the intervention was effective only among them. Overall, the intervention arm contributed to $14 \%$ of the bacteriologically confirmed PTB case detection and the impact of expansion of the intervention to the control arm, thus covering all eligible PPs in the city, could increase the yield to a quarter or so of all cases. This resonates an opinion piece from India arguing for the potential of solo PPs in PPM [22]. In Myanmar, the contribution of PPs to bacteriologically confirmed PTB case detection was $20 \%$ [23]. In Pakistan in 2015, solo GP clinics contributed $17 \%$ of the bacteriologically confirmed PTB cases detected in PPM facilities [24].

The proportion of bacteriologically confirmed PTB cases among individuals referred by study PPs was high in both arms and $50 \%$ had grade 3 positive sputum, suggesting selective referrals. Whether there was PP delay in referral or patient's delay in seeking care cannot be ascertained, but the programme should encourage PPs to refer individuals with presumptive TB and not only cases or individuals with a high prior probability of TB.

There is growing experience in the use of digital technologies to support TB elimination, especially regarding 
improving treatment adherence and notification [25]. In this study, regular feedback about referred cases through SMS was used as a means to strengthen communication between PPs and RNTCP. Monthly visits and the assurance that PPs could retain their patients for further treatment if they wished, possibly gave them confidence and increased their motivation to refer.

Only few PPs notified cases put on private treatment after diagnosis outside RNTCP. Raising awareness, collection of notification forms right at PP clinics and constant dialogue between RNTCP staff and PPs had no effect on notification in our study. Studies trying to understand the barriers to notification in India reported that not all PPs have fully understood the notification's rationale and that, although aware, many do not notify, the main reasons being lack of time, concerns regarding patients' confidentiality and lack of trust in the government health system $[15,26,27]$. Ensuring notification without legal backup seems difficult. A mix of collaboration and regulation might help. For example, the clinical establishment act [28], setting the minimum standards for accrediting private health facilities, could be used as an enforcement tool.

A novel aspect of this study is the central role played by TB-Health Visitors in linking PPs and RNTCP, a strategy largely underexploited but that proved successful in a similar setting $[29,30]$.These cadres are essentially outreach workers ensuring treatment adherence, who are expected to act as interface with the private sector but do not receive any formal training. In our study, TB-Health Visitors were trained on how to interact with PPs and, while establishing a trust relationship with them took time, it was eventually successful. Identification cards affiliating TB-Health Visitors to RNTCP also eased their access into PPs' health facilities and, not of less importance, gave them motivation. Notwithstanding, the role of the DTO was crucial for the partnership. He conducted ad hoc visits to PPs clinics and was always available on the phone to discuss cases. In the perspective of scale-up, this role could be shared with the Medical Officer-TB control and PPM coordinator, when available.

We successfully involved PPs without providing financial incentives. Provision of financial incentives for engaging PPs in PPM has been widely advocated and put in practice with varied results. Some argued this would jeopardise the partnership, prove unsustainable in the long run and be difficult to replicate $[21,31]$. In Vietnam, financial incentives increased PPs referrals, but $58 \%$ of the referred cases defaulted before initiating treatment [32]. Financial incentives come with inherent challenges of record verification and transparency. In the absence of a strong regulatory mechanism in the country [33], our approach was primarily based on building good relationship, strong communication and persuasion.

There have been suggestions to use local NGOs as public-private intermediary agencies (PPIAs) in PPM activities $[34,35]$. There is a fair amount of information on PPIAs in different countries such as Bangladesh, Cambodia and India. But a pragmatic question to be answered is whether such human and financial resource-intensive approach is scalable with domestic funding. Rather, we suggest enhancing national TB programme (NTP) and general health system staff capacity to take up the function of intermediary agency. In our study, research activities were embedded within RNTCP and staff capacity was built by default. However, the DTO hesitated to formally involve PPs in the PPM schemes, because of limited funds under PPM. Further, the field staff expected additional funds for travel expenses to meet PPs, which was diverted from contingency funds available with DTO. It is necessary to ensure sufficient financial and human resources for setting up PPM schemes. Currently, these are not available [36]. The interaction between the private and public sector is limited also by conflicting interests and mutual mistrust $[37,38]$. The fundamental question to be answered is whether the public sector is equipped to negotiate, build and sustain the relationship with the private sector. The public sector may not have full capacity and willingness to engage with the private sector, specifically when facing scarce resources and weak governance $[39,40]$. However, PPM needs a strong and motivated public sector [41].

Lastly, RNTCP lacks meaningful targets and indicators for PPM activities, especially regarding PPs, whose referrals are neither recorded nor reported [10]. Systematic monitoring and evaluation of PPM should be institutionalised.

In conclusion, a system-oriented intervention based on the establishment of a strong referral and communication system with PPs practising in solo clinics and nursing homes improved the involvement of GPs and internists in referral of presumptive PTB cases to RNTCP. This study, conducted in a routine programmatic setting, provides empirical evidence on how to use low-cost interventions built within NTPs and mobilise the potential of all its cadres, in order to increase the collaboration of PPs with the NTP. The model can be the first building block for the design of additional interventions to further improve the collaboration of formal allopathic PPs with RNTCP, as well as be extended with specifically tailored interventions to improve, if and where relevant and meaningful, the collaboration with non-allopathic and informal private providers. It could also inspire other 
health and disease control programmes and be adopted on a wider scale in general health systems. Based on the outcomes of this study, the intervention is now extended to the control arm PPs and rolled out and evaluated in a rural part of the same district [42].

\section{Acknowledgements}

The authors thank the RNTCP staff who implemented the project and the PPs who participated in the study. This study was funded through a framework agreement between the Institute of Public Health (Bangalore), the Institute of Tropical Medicine (Antwerp) and the Belgium Directorate-General for Development Cooperation.

\section{References}

1. World Health Organization. Global tuberculosis report 2016. World Health Organization: Geneva, Switzerland, 2016.

2. World Health Organization. Engaging all health care providers. Guidance on Implementing Public-Private Mix Approaches. World Health Organization: Geneva, Switzerland; 2006.

3. Uplekar M. Involving private health care providers in delivery of TB care: global strategy. Tuberculosis 2003: 83: 156164.

4. Auer C, Lagahid JY, Tanner M, Weiss MG. Diagnosis and management of tuberculosis by private practitioners in Manila, Philippines. Health Policy (New York) 2006: 77: 172-181.

5. Satyanarayana S, Subbaraman R, Shete P et al. Quality of tuberculosis care in India: a systematic review. Int J Tuberc Lung Dis 2015: 19: 751.

6. Jaroslawski S, Pai M. Why are inaccurate tuberculosis serological tests widely used in the Indian private healthcare sector? A root-cause analysis. J Epidemiol Glob Health 2012: 2: $39-50$,

7. Sreeramareddy CT, Qin ZZ, Satyanarayana S, Subbaraman R, Pai M. Delays in diagnosis and treatment of pulmonary tuberculosis in India: a systematic review. Int J Tuberc Lutng Dis 2014: 18: 255-266.

8. Tanimura T, Jaramillo E, Weil D, Raviglione M, Lonnroth $\mathrm{K}$, Lönnroth K. Financial burden for tuberculosis patients in low- and middle-income countries: a systematic review. Eur Respir J 2014: 43: 1763-1775.

9. Udwadia ZF, Pinto LM, Uplekar MW. Tuberculosis management by private practitioners in Mumbai, India: has anything changed in two decades? PLOS ONE 2010: 5: 1-5.

10. Yellappa V, Battaglioli T, Gurum SK, Narayanan D, Van Der Stuyft P. Private practitioners' contributions to the Revised National Tuberculosis Control Programme in a South Indian district. Int J Tuberc Lutg Dis 2016: 20: 659-665.

11. Dewan PK, Lal SS, Lonnroth $\mathrm{K}$ et al. Improving tuberculosis control through public-private collaboration in India: literature review. BMJ 2006: 332: 574-578.
12. Mahendradhata $Y$, Lambert M-L, Boelaert M, Van der Stuyft P. Engaging the private sector for tuberculosis control: much advocacy on a meagre evidence base. Trop Med Int Health 2007: 12: 315-316.

13. Khan MS, Salve S, Porter JDH. Engaging for-profit providers in TB control: lessons learnt from initiatives in South Asia. Health Policy Plan 2015: 30: 1289-1295.

14. Directorate of Health and Family Welfare Government of India Central TB Division. Guidance for TB Notification in India; 2012.

15. Philip S, Isaakidis P, Sagili KD, Meharunnisa A, Mrithyunjayan S, Kumar AMV. "They Know, They Agree, but They Don't Do"- the paradox of tuberculosis case notification by private practitioners in Alappuzha District, Kerala, India. PLOS ONE 2015: 10: e0123286.

16. Yellappa V, Lefèvre P, Battaglioli T, Narayanan D, Van der Stuyft P. Coping with tuberculosis and directly observed treatment: a qualitative study among patients from South India. BMC Health Serv Res 2016: 16: 283.

17. Yellappa V, Lefêvre P, Battaglioli T, Devadasan N, Van Der Stuyft P. Patients pathways to tuberculosis diagnosis and treatment in a fragmented health system: a qualitative study from a south Indian district. BMC Public Health 2017: 17: 635.

18. Lei X, Liu Q, Escobar E et al. Public-private mix for tuberculosis care and control: a systematic review. Int J Infect Dis 2015: 34: 20-32.

19. Sharma SK, Mohan A, Chauhan LS et al. Contribution of medical colleges to tuberculosis control in India under the Revised National Tuberculosis Control Programme (RNTCP): lessons learnt \& challenges ahead. Indian J Med Res 2013: 137: 283-294.

20. Murthy KJR, Frieden TR, Yazdani A, Hreshikesh P. Publicprivate partnership in tuberculosis control: experience in Hyderabad, India. Int J Tuberc Lung Dis 2001: 5: 354-359.

21. Khan AJ, Khowaja S, Khan FS et al. Engaging the private sector to increase tuberculosis case detection: an impact evaluation study. Lancet Infect Dis. 2012: 12: 608-616.

22. Lal SS, Sahu S, Wares F, Lönnroth K, Chauhan LS, Uplekar M. Intensified scale-up of public-private mix: a systems approach to tuberculosis care and control in India. Int J Tuberc Lung Dis 2011: 15: 97-104.

23. Nwe TT, Saw S, Le Win L et al. Engagement of public and private medical facilities in tuberculosis care in Myanmar: contributions and trends over an eight-year period. Infect Dis Poverty 2017: 6: 123.

24. Khan BJ, Kumar AMV, Stewart A et al. Alarming rates of attrition among tuberculosis patients in public-private facilities in Lahore, Pakistan. Public Health Action 2017: 7: 127 133.

25. Nglazi MD, Bekker L-G, Wood R et al. Mobile phone text messaging for promoting adherence to anti-tuberculosis treatment: a systematic review. BMC Infect Dis 2013 Dec 2: 13: 566 .

26. Thomas BE, Velayutham B, Thiruvengadam K et al. Perceptions of private medical practitioners on tuberculosis 
notification: a study from Chennai, South India. PLOS ONE 2016:11:e0147579.

27. Yeole RD, Khillare K, Chadha VK, Lo T, Kumar AMV. Public Health Action Tuberculosis case notification by private practitioners in Pune, India: how well are we doing? Public Heal Action 2015: 5: 173-179.

28. Government of India: Ministry of Law and Justice (Legislative Department). Clinical Establishments (Registration and Regulation) Act 2010. Nr23 of 2010. New Delhi; 2010.

29. Pradhan A, Datye V, Kielmann K et al. Sustaining PPM DOTS: the case of Pimpri Chinchwad, Maharashtra, India. Indian J Tuberc 2010: 58: 18-28.

30. Kielmann K, Datye V, Pradhan A, Rangan S. Balancing authority, deference and trust across the public-private divide in health care: tuberculosis health visitors in western Maharashtra, India. Glob Public Health 2014: 9: 975-992.

31. Zafar Ullah AN, Huque R, Husain A, Akter S, Islam A, Newell JN. Effectiveness of involving the private medical sector in the National TB Control Programme in Bangladesh: evidence from mixed methods. BMJ Open 2012: 2: e001534.

32. Quy HT, Lan NTN, Lönnroth K, Buu TN, Dieu TTN, Hai LT. Public-private mix for improved TB control in Ho Chi Minh City, Vietnam: an assessment of its impact on case detection. Intern J Tuberc Lung Dis 2003: 7: 464-471.

33. Sheikh K, Saligram PS, Hort K. What explains regulatory failure? Analysing the architecture of health care regulation in two Indian states. Health Policy Plan 2015 Dec: 30: 3955 .

34. Lönnroth K, Uplekar M, Arora VK et al. Public-private mix for DOTS implementation: what makes it work? Bull World Health Organ 2004: 82: 580-586.
35. Hurtig AK, Pande SB, Baral SC, Newell J, Porter JDH, Bam DS. Linking private and public sectors in tuberculosis treatment in Kathmandu Valley, Nepal. Health Policy Plan 2002: 17: 78-89.

36. Lal SS, Uplekar M, Katz I et al. Global Fund financing of public - private mix approaches for delivery of tuberculosis care. Trop Med Int Health 2011: 16: 685-692.

37. Vyas RM, Small PM, Deriemer K. The private-public divide: impact of conflicting perceptions between the private and public health care sectors in India. Int J TB Lung 2003: 7: 543-549.

38. Salve S, Sheikh K, Porter JDH. Private practitioners' perspectives on their involvement with the tuberculosis control programme in a Southern Indian state. Kerman Univ Med Sci 2016: 5: 631-642.

39. Probandari A, Utarini A, Lindholm L, Hurtig A-K. Life of a partnership: the process of collaboration between the National Tuberculosis Program and the hospitals in Yogyakarta, Indonesia. Soc Sci Med 2011: 73: 1386-1394.

40. Rangan SG, Juvekar SK, Rasalpurkar SB, Morankar SN, Joshi AN, Porter JDH. Tuberculosis control in rural India: lessons from public-private collaboration. Intern J Tuberc Lung Dis 2004: 8: 552-559.

41. Mahendradhata Y, Lambert M-L, Van Deun A, Matthys F, Boelaert M, van der Stuyft P. Strong general health care systems: a prerequisite to reach global tuberculosis control targets. Int J Health Plann Manage 2003: 18(Suppl 1): S53S65.

42. World Health Organization. TDR / Second round of IMPACT Grant awardees announced [Internet]. TDR. World Health Organization; 2015 (Available from: http:// www,who.int/tdr/news/2015/impact-grant/en/) [19 Jul 2017]

Corresponding Author Vijayashree Yellappa, Institute of Public Health, Bangalore, India. Tel.: +91 981830 7814;

E-mail: vijayashree@iphindia.org 


\section{PART IV - GENERAL DISCUSSION}

This section summarises and brings together results from the three sub-studies. It discusses the implications of the major study findings in relation to the existing literature.

Our study demonstrated the effectiveness of the health system oriented multi-component intervention package integrated into RNTCP. The intervention was effective at improving the involvement of PPs (general practitioners and internists) in referral of presumptive pulmonary TB cases for sputum examination to RNTCP. During the study period, PPs referred 836 patients having symptoms of pulmonary TB cases, out of which 176 were diagnosed with bacteriologically confirmed pulmonary TB. The proportion of referring PPs, mean referral and smear positive TB case finding rate per PP-year were significantly higher in the intervention than the control arm. Strength of our study is that we gathered the evidence from the field to build a multicomponent intervention by carefully studying the contextual factors, which was later refined in consultation with stakeholders.

\section{ASSESSMENT OF LOCAL PRE-CONDITIONS FOR PARTNERSHIPS}

Despite the abundance of literature describing PPM experiences, robust empirical evidence of their effectiveness on programme outcomes is limited. Studies have shown that there is much variation in the process of building partnerships and the nature of services for which partnerships were built (Mukund Uplekar, Pathania, and Raviglione 2001), (Dewan et al. 2006). There have been arguments indicating that the existing evidence for PPPs does not allow for easy generalisations (Planning Commsion 2012) and that the success of public health interventions is mostly context dependent (Minary et al. 2018). It is therefore essential to understand how context and intervention systems interact and identify what needs to be replicated as interventions in different contexts.

With this background in mind, we carried out a systematic situational assessment during the preconditions phase (2012-2013) and pre-implementation phase (2013-2014). to assess the local preconditions available for partnerships. Our initial assessment of the field situation revealed the lack of coordination in implementing the PPM activities in the study district. We also found that there was lack of clear purpose, leadership and commitment in the public sector to engage with PPs. Further, engagement of PPs in TB care and control did not seem to be a priority for the programme. In fact, PPM was reduced to a loose co-existence of the two sectors, with no accountability on either side. PPM approach was mostly based on a voluntary basis, depending on the interest a PP holds to 
collaborate with RNTCP. Significant differences in perception were found between the two sectors, which resulted in mistrust and conflicting mind-sets, very much in line with other findings (Vyas, Small, and Deriemer 2003), (De Costa, A., Johansson, E., \& Diwan 2008). These findings helped the research team to understand and assess, what would work best in a given situation, and how interventions are to be executed. In fact, Simpson (Simpson 2002) while proposing the conceptual framework for transferring research into practice debated that several organisational considerations such as institutional readiness for change, resources, staff attributes and as well as culture) needs to be considered when planning an implementation of evidence-based interventions. Hence, by acknowledging the need for a thorough understanding of the context, and carefully understand the existing pre-conditions to inform the conception and the execution of the intervention in the study area, we carried out series of activities assess the pre-conditions in place for partnerships, and assess the likelihood of success of our intervention.

In the next paragraphs, we elaborate on the activities that were carried out to as part of pre-condition phase:

\subsection{Systematic mapping of PPs}

For a successful execution of a PPM strategy, the first and foremost step is to know the private sector landscape with whom the public sector wants to engage with. This assessment has been recognised as a prerequisite for an effective PPM strategy (World Health Organization 2007); this is especially relevant in India with its pluralistic private health sector. Carrying out this exercise is not straightforward, given the heterogeneous health-care landscape in India. In fact, poor information on the private sector has been recognised as one of the major barriers of PPP (Harding 2009). In the study area, there was no single registry which contained the total number of PPs practicing in the district. In order to get an idea of the number of PPs practicing in the district, we searched several data sources in the study area. However, none of the data source was complete. For example, the list of PPs available in the district TB office contained only $40 \%$ of the PPs that we ultimately mapped.

Currently, there are no operational guidelines for mapping PPs in RNTCP. The available WHO guidelines to engage PPs provide only broad principles of engagement, without the practical guidance to carry out such a mapping exercise of the private sector. Thus, there is need to develop a training manual by RNTCP. In our study, it was essential to identify the characteristics of PPs and understand their capacity to undertake the tasks envisaged in the PPM strategy to engage with RNTCP. Therefore, we undertook a census of allopathic for-profit PPs in the study area, which indeed was time and resource-intensive. We faced several challenges in mapping the PPs: the majority of general practitioners in our study practiced in stand-alone clinics, which were more challenging to identify and locate; specialists worked in more than one health facility, and some others visited the city once in a week/fortnight. Similarly, mapping of DOT providers and of microscopic centres in 
the public sector along with contact details of medical officers and laboratory technicians in charge of microscopic centres was carried out. As an output, a directory containing the contact details of PPs and key RNTCP staff was developed to improve the communication and thus the collaboration.

Thus, considering the dynamic character of the private sector, a systematic mapping of PPs should be carried out periodically by local health administrations, since the utility of this exercise goes well beyond the sole TB programme (West Slevin, Forbes, and Wells 2014). We suggest that a register be maintained at the primary health centre which captures the database of PPs practicing in the area, and which should be accessible to the programme managers. This database should eventually lead to the setting up of a national database on PPs practicing in different parts of the district to facilitate collaboration beyond TB. This information could be used for engaging PPs for other national health programmes as well.

\subsection{Recording and Reporting of Patient's Referrals from PPs to RNTCP}

As a first step in developing a PPM initiative, it is essential to assess the current level of PP's involvement in the TB programme. Programme operational guidelines (Central TB Division; Government of India. 2016) recommends that the source of referral and the place of treatment should be routinely recorded and reported to assess PP's participation in national TB programme (World Health Organization 2006). However, in the study area PP's contribution to RNTCP was not routinely measured and reported in the programme. Laboratory technicians stationed at the microscopic centres doing sputum microscopy did not routinely record the names of the referring PPs in the laboratory register and feedback was seldom given to the referring PPs. Since PPM activities were not documented systematically, RNTCP staff did not report private sector TB related performance. This is why, despite the wealth of experience from numerous evaluations of small to medium sized PPM initiatives in India, measurement of PPM contribution on a national scale has not been possible in most settings. This concern was also reported in the WHO evaluation report (Aime De Muynck, David Berger, Tim Clary, Selva Kumar 2011).

Further, the PPM section of RNTCP quarterly reports lacks meaningful key performance indicators to measure PPM activities. The PPM section provides only the absolute number of PPs involved in any PPM scheme, without any disaggregated data according to different types of health care providers to estimate the contribution of PPs to RNTCP. Therefore, there is an absolute need to introduce a range of PPM performance indicators that are realistic, reliable and verifiable in RNTCP quarterly report (Mukund Uplekar, Pathania, and Raviglione 2001).

Considering these lacunae in PPM reporting system, the first and foremost step that we took in our study was to fix and strengthen the documentation of source of referrals in microscopic centres' laboratory registers. Separate trainings were conducted for laboratory technicians to emphasise the importance of documenting the source of referrals and name of the referring PPs. A list of PPs 
practicing in the area was provided to laboratory technicians to facilitate the recoding the names of referring PPs. We designed simple PPM tools for different cadres of RNTCP staff involved with PPM activities, to capture PPM activities. These tools were refined and adapted as the project evolved. A consolidated PPM report was prepared by the PPM coordinator on a monthly basis and was monitored by District TB Officer/ MO-TB Control in PPM meeting held at the end of every month.

\subsection{Patient's Therapeutic Itineraries to RNTCP \& PP's Cross Referral Practices}

In India, accessing care and adhering with the long course of TB treatment is a complex endeavour (Charles et al. 2010), (Nimbarte, Wagh, and Selokar 2011), (Isaakidis et al. 2013), (Rajesh D Deshmukh, Dhande, and Sachdeva 2015), (Kapoor et al. 2012). Our qualitative study with patients revealed that they resorted to different modes of care; self-medication by taking on counter drugs from retail private pharmacists, a second group sought care from government facilities and had simple itineraries. A third group, who sought care from PPs, switched concurrently and/or iteratively between public and private providers in search for relief of symptoms causing important diagnostic delays. PP's cross-referral practices to RNTCP were influenced by patient's paying capacity, their familiarity with RNTCP, kickbacks from private labs and chemists, and in some cases to simply get rid of TB patients for the fear of spreading the infection in their health facility. These trade-offs by PPs complicated patient's itineraries to RNTCP, and resulted in poor quality TB care, with disastrous consequences for patients. Considering these loopholes, we designed a number of interventions to ease the flow of patients to RNTCP, such as provision of sign boards at the reception of government hospitals, training of receptionists to guide patients to laboratory without making them stand in the ques, ensuring the availability of laboratory technicians at microscopy centres, provision of maps showing directions to the microscopic centres, and printing the contact details of laboratory technicians behind the referral slips. This was found useful by PPs in directing the patients to microscopic centres.

\section{TYPE OF PROVIDERS PRIORITISED FOR ENGAGEMENT}

Most of the past successes of PPM approaches have come from the engagement with large hospitals and medical colleges (Sharma et al. 2013; A. J. Khan et al. 2012; Murthy et al. 2001), which are more likely to yield a higher number of patients, in contrast to PPs practising in stand-alone clinics. Many PPs having stand-alone clinics do have neither the infrastructure nor the managerial capacity to sign for PPM schemes other than the referrals of TB patients to RNTCP. Unfortunately, PPM schemes under RNTCP offered limited options to individual PPs to get engaged with RNTCP in terms of referrals of presumptive TB patients to RNTCP (Central TB Division, Directorate general of health services 2014). PPM remained as a loose co-existence of the two sectors, with no accountability at either side. 
We noted that a large number of patients select General Practitioners (GPs) as first-line providers and GPs tend to refer substantial number of patients to RNTCP. Our study findings suggest that some PPs are worth prioritising compared to others; GPs and internists (both working on an ambulatory out-patient basis in the community and hospital based) had a higher volume of referrals and the intervention was effective in this category of PPs. Another study conducted in India also pointed to a substantial contribution by individual PPs from 14 cities which had intensified PPM activities (Lal, Sahu, et al. 2011b). Another study also resonated that GP's involvement is of particular importance in reducing diagnostic delay and save costs for patients (Pantoja, Lönnroth, et al. 2009). Similar findings were reported from other high TB burden countries, such as Nepal and Vietnam as well (Maung et al. 2006, Pethani et al. 2015, Caminero and Billo 2003), (Nwe et al. 2017). Hence, involvement of GPs providing primary care in the solo clinics needs to be prioritised for engagement to provide standardised care for TB patients. Partnerships should therefore be based on criteria that can help prioritise engagement with potential PPs who are most likely to contribute to RNTCP. Our study shows that an introduction of a PPM scheme specifically to facilitate referrals of presumptive pulmonary TB patients from individual PPs to RNTCP may increase PP's involvement in the programme.

In our study, we included only for-profit allopathic PPs. However, findings from patients' interviews revealed that patients with TB symptoms often start with seeking advice from chemists and unqualified practitioners, in line with the findings of another study documenting the trajectory of TB patients in the TB care landscape (Kapoor et al. 2012). Other studies have shown that AYUSH providers practicing allopathic medicine are the first contact points for seeking care, especially so in rural India (May, Roth, and Panda 2014) and they stressed the need to engage them in PPM efforts (Achanta et al. 2013). However, it should be borne in mind that the informal private sector in India remains on the margins of institutional frameworks (Pinto, 2004; Gautham, 2011), and thus poses a challenge in engaging them in national health programmes (Cross and Macgregor 2009). Possibly, RNTCP could involve AYUSH providers to undertake activities such as referrals of presumptive TB patients, provision of DOTS, promote treatment adherence among TB patients, and increase community awareness about TB. Nevertheless, this area requires further research to understand the mechanisms to operationalise the systematic involvement of informal providers in the programme.

\section{BUILDING PARTNERSHIP BETWEEN PPS \& RNTCP}

We hypothesised that PPM programme requires investment into both the public and private sectors, as suggested by other PPM studies (Mahendradhata et al. 2007a). Studies have cautioned that if strengthening of both sectors are not carried out, it would be a threat for the sustainability of the partnership (Pradhan et al. 2011a) (De Costa, A., Johansson, E., \& Diwan 2008). Therefore, we used a number of best practices identified in the literature, supplemented with our own empirical evidence to design the intervention package and build the partnership. 
We took directions from the REP framework (Kilbourne et al. 2007) to implement the intervention in the study setting. While we used the basic principles of REP model to implement the intervention, certain activities in the framework were modified based on our project needs. The REP framework allowed for such flexibility. However, we realised that the conceptual framework cannot be applied completely, and that it is important to customise the framework to implement intervention. This is because, the pre-conditions available for building partnerships and the local context of general health system are unique to each study setting. we continuously made efforts to adapt the framework to local health system dynamic contexts. We found processes such as continuous engagement with different stakeholders, timely identification and minimising the constraints for effective implementation of interventions, feedback mechanisms that facilitated building the trust were crucial for successful adaptation of the framework. Also, the learning was, it is better to bundle the interventions through packaging of multiple interventions, rather than single component strategy to have an impact of the intervention. Further, scaling up of successful interventions requires tailoring of strategies to the various dimensions of the study settings and that the model cannot be replicated as it is. Researchers who worked on the frameworks of scaling up of health interventions (Simmons, Ruth, Peter Fajans 2007) (Minary et al. 2018) have also shown that no single strategy or blueprint exists to strengthen health services in developing countries.

In the coming sections, we describe the activities that were carried out to initiate, implement and sustain the partnership.

\subsection{Designing the Intervention Package}

The intervention package was developed and revised in close coordination with RNTCP staff and leading PP's suggestions. Some proposals made by PPs were the following: provision of sputum cups at PP's clinics so that patients could collect the early morning sputum sample and reach microscopic centres for sputum examination, provision of referral slips and notification forms and provision of contact details of key RNTCP staff, etc. Regular consultative meetings were held with RNTCP staff to solve the operational challenges in building partnerships with PPs. Individual meetings were organised with PPs who had a good 'clientele' but did not refer patients to RNTCP. These meetings proved to be critical in building trust among PPs early in the study and to clear the misconceptions about RNTCP services and establish shared values and alignment of purpose.

Creating a partnership was not without challenges, but it was a continuous learning process, with unexpected lessons, which necessitated mid-course corrections: (i) over half of the intervention PPs did not participate in the initial training. Hence, the District TB Officer and his team, along with the PI, had to visit individual PPs personally to explain them the project and secure their participation; (ii) PPs tend to admit their patients in private nursing homes where patients were managed by duty doctors (fresh medical graduates) and nurses, and they were responsible for the actual execution of PPs' referrals and notification of patients to RNTCP. Realising their important role in TB care, a 
separate training was organised as a mid-course correction to train this cadre of personnel working in private nursing homes; (iii) TB-Health Visitors had to wait several hours to meet busy PPs or were not allowed by receptionists to meet PPs. Therefore, we developed visiting cards for TBHealth Visitors with a logo of the department of Health and Government of Karnataka. This small intervention eased TB-Health visitor's entry into the busy clinics and nursing homes.

\subsection{Training of PPs and the Key RNTCP Staff in PPM Strategy}

Our predisposition was that a strong public health system is a pre-requisite for success of the PPM strategy. The public sector has to lead by example and must be willing to implement, supervise and evaluate PPM projects. This opinion was also echoed by another study which analysed the processes and outcomes of four PPM projects in high burden countries (Lönnroth et al. 2004). Evidence showed that the PPM models that could not produce the desired results, mainly suffered from the lack of firm commitment from the government, poor monitoring and supervision, and selection of inappropriate partners (Arfina, n.d.). Rama Baru (Baru and Nundy 2008), while analysing the complexity of PPP in health care, argued that a strong public health system in terms its capacity to manage partnerships is a prerequisite for success of PPP. Further, Dewan et al (Dewan et al. 2006) while analysing PPP pilots in India reported that a strong public sector TB control programme is critical for the provision of necessary advocacy, training, and supervision.

Studies have shown that the governments in many low and middle income countries lack the essential skills, tools and guidelines to develop and maintain PPPs effectively (Harding 2009),(Mills et al. 2002). It is generally assumed that public managers can carry out new policy initiatives regardless of the behavioural, cognitive or technical demands that the introduction of such policies may make upon them. A study showed that in effect, public managers have to learn a range of often new techniques in order to implement what are often ambiguous policy directives (Jill Schofield 2004). Our assessment of the pre-conditions in place for building a partnership showed that RNTCP staff had little capacity to negotiate with PPs to convince them to refer patients to RNTCP. They made attempts to collaborate only with those PPs who agreed to follow the standard treatment guidelines prescribed by RNTCP. Therefore, PPs who did not refer or notify patients to RNTCP were automatically excluded and their potential thus not exploited by RNTCP. Field staff expressed the need for regular training on PPM to enhance field staffs' ability to interact with PPs. Hence, training was made a top priority in our project. We undertook a series of trainings for different cadres of RNTCP, starting from laboratory technicians to strengthen the documentation of PP's referrals, counselling the patients to go back to referring PPs to show the laboratory results, sending SMS to the referring PPs about the laboratory results, and consolidating and reporting PP's referrals every month etc. Hands on training was given to TB-Health visitors on how to negotiate with PPs to convince them to refer patients to RNTCP and make regular visits to PP's clinics to give feedback on the referred patients and collect the notification of new patients on private treatment. RNTCP staff were trained to document and report PPM activities. A supervisory committee consisting of the 
District TB Officer and MO-TB Control supported by the PI looked into the day-to-day operational issues. These strategies helped in establishing clear standards for implementing PPM activities and constantly refining the process to make the system more efficient.

Consequently, we trained PPs in the intervention arm of our intervention. Our assessment of the pre-conditions for partnership conducted through interviews with PPs revealed a number of factors that would hinder PP's participation in RNTCP. For instance: lack of feedback from RNTCP when patients are referred, fear of losing credibility when RNTCP did not respect PP's clinical acumen, high turnaround time for sputum test results coupled with poor quality of chest X-ray, unavailability of comprehensive TB care, and inherent problems with DOTS delivery which compromised the patient's privacy. PPs were also dubious about the quality of services available under RNTCP, which contributed to their reluctance to refer patients to RNTCP. PPs were reluctant to maintain any document related to TB patients, including signing a MoU with RNTCP, similarly to other study findings ( Muynck, Berger, Tim Clary, Kumar 2011). Training was adapted to PP's needs in terms of timing, date and the content. Training was used as an opportunity to clear the PP's concerns about RNTCP. We utilised 'leading' PPs, who were mostly the senior doctors having popularity in the medical fraternity in to influence PPs who were not willing to engage with RNTCP. Some of the concerns surrounding quality issues were discussed. The State TB officer, who was invited as resource person himself was a physician. He took lead in clarifying the doubts of PPs. Further, the objective and the benefits of referring and notifying patients to RNTCP were explained. Standards for TB care in India developed in a booklet (Organisation 2014) and other advocacy tools were provided to PPs. RNTCP field staff implementing the PPM were introduced to the PPs during this event. However, only 50\% of intervention PPs attended the government training. This area requires further research in order to understand the reasons for this low attendance.

\subsection{Building the Capacity of RNTCP Field Staff as An Interface of PPM Strategy}

TB-health Visitors (TB-HVs) played a crucial role in negotiating and sustaining partnerships with the involved PPs in RNTCP. TB-HVs are outreach workers, under the payroll of RNTCP, to ensure patient treatment adherence through advocacy and education by providing DOTS at public facilities. But, in the programme, they are expected to act as an interface with the private sector, sensitise PPs and involve them in RNTCP, without any formal training in PPM approach, yet expected to carryout PPP activities. The regular feedback provided by TB-HVs through monthly visits to PPs clinics to provide referral slips and collect notification forms proved useful in building trust among PPs.

Sadly, TB-HVs role remain undervalued in the system and they are nowhere recognised as genuine negotiators of PPM. A study examining the role of TB-Health Visitors, reported that their potential within PPM remains undervalued (Kielmann et al. 2014), because accountability is measured through technical rather than social outcomes of the 'partnership' (Kielmann, Datye, and Pradhan 
2014). We observed that TB-HVs found it difficult to fulfil both programme directives as per their Terms of Reference and implement PPM activities, similarly to other study finding (Pradhan et al. 2011b). PPM activities did not offer incentives and did not contribute to TB-HV's measurable performance. Hence, the programme will have to revisit the role of TB-HVs working relationships within the structures of RNTCP vis-à-vis PPM and amend their Terms of Reference and training needs accordingly.

Evaluation of RNTCP by USAID (Aime De Muynck, David Berger, Tim Clary, Selva Kumar 2011) and other study by Nora Engel (Nora Engel 2013) reported that PPs resented the supervision by field RNTCP staff (TB-Health Visitors /Senior Treatment Supervisor/Senior TB Laboratory Supervisor) as they were perceived as staff of a lower professional status, because they were non- medical personnel. We found that establishing the initial contact with PPs best happens when either the MOTB or the district TB officer is involved in establishing the initial contact with PPs and/or resolve the issues themselves instead of leaving it to the field staff.

\subsection{Critical Need of Communication to Build Partnerships}

A significant challenge was faced while initiating partnership and to build understanding and trust among PPs. This was particularly challenging with PPs who were either not familiar with RNTCP or not in the favour of collaborating with RNTCP. We found significant conflicting perceptions between the private and public health care sectors such as lack of trust and mutual understanding, long turnaround time for sputum examination, problems with effectiveness in the DOTS delivery, technical incompetence of medical staff in government facilities to deal with drug toxicity, and bureaucratic hurdles in disbursement of financial incentives, etc., which confirmed other study findings (Costa et al. 2008), (Vyas, Small, and Deriemer 2003), (Muraleedharan et al. 2005), (Uplekar et al 2001). Engel et al, while documenting PPM as organisational innovation in India reported that building collaboration is often difficult and argued that it is critical to mitigate the apprehensions between professions to make organisational PPM, sustainable and scalable (Engel and van Lente 2013). Other studies echoed that communication is key for collaboration and stressed that a partnership can be established if it begins with an intensive process of interaction and communication at the individual level to build trust between programme staff and PPs (Probandari A, Utarini A, Lindholm L 2011), (Watkins et al. 2006), (Ambe, Lönnroth, Dholakia, Copreaux, Zignol, Borremans, Uplekar, et al. 2005). A study which studied PP's perspective of engaging with RNTCP reported that, though PPs felt responsible for their TB patients, they found it difficult to perceive themselves as 'partners with the TB programme' (Salve, Sheikh, and Porter 2016).

Considering the above issues, we designed a bundle of interventions to improve the communication between PPs and RNTCP, which eventually yielded fruitful results. Our intervention consisted of providing referral slips with carbon copies and the protocol for sending SMS to PPs about patient's sputum results to PPs gave them confidence that they would not lose their patients, which greatly 
increased their motivation to remain in the partnership. Additionally, regular feedback about the patients referred by PPs through monthly visits of TB-Health Visitors to PP's clinics and the assurance that they could retain their patients for further treatment if they wished so, also gave PPs confidence and increased their motivation to refer. Mistrust was replaced by a constructive partnership. PPs started making phone calls to TB-HVs when they referred patients to microscopic centres. In this regard, provision of RNTCP staff's phone directory was found to be useful. The engagement strategy that emerged from our study reflects an underlying need for building greater trust and better relationships between the public and private health sectors through consistent communication. Therefore, communication should be planned and carried out as an integral part of the programme.

\subsection{Maintaining Partnership}

Building and maintaining partnership was indeed a time and resource-intensive endeavour, which demanded a strong and continuous commitment from the public sector, especially in a scenario where the private sector lacked trust and interest in public health aspects of TB management. Other studies have shown that the creation and development of strategic partnerships is a difficult and a dynamic process (Buse \& Harmer, 2007), (R.Reich 2006), (Widdus, 2001a) and investing time and effort is needed to ensure that sufficient dialogue takes place among all stakeholders, build trust and achieve agreement (Lönnroth et al. 2004). At the end of the project, the majority of the intervention PPs routinely started referring the patients in their daily practice, without any active involvement from the field RNTCP team. Also, the PPM reporting formats developed for research purpose got integrated into routine monitoring activities. Programme managers started using these formats to review the involvement of PPs in the project. As existing partnerships become more established, it required fewer inputs from the PI to maintain them.

Our project differs from other PPM projects in terms of sustainability, because RNTC staff carried out the research activities, and their capacity to collaborate with the private sector was gradually built during the study period. Further, clear demarcation of roles and responsibilities of RNTCP contributed to liaising PPM activities at different levels. The required leadership to foster communication between the partners was provided by the District TB Officer. This demonstrated the administrative commitment available in the study setting to build the partnership. In a nutshell, working in a consultative mode with influential PPs for obtaining endorsements of the intervention package, developing contextually appropriate strategies, constant communication with key stakeholders and supportive supervision of the field RNTCP staff, contributed in building the maintaining the partnership. However, it is imperative that DTO invest sufficient time in interacting with PPs to educate them about RNTCP. But in reality, we found that the programme management structure at both district and sub-district levels continue to burden the programme managers with administrative functions leaving them with little or no time for supervisory and monitoring activities. 


\section{BUDGET ALLOCATION FOR PPM ACTIVITIES UNDER RNTCP}

Results-based financial incentivising has been the operational model of the current PPM schemes. Making PPM work clearly requires significant resources, time and energy, which incurs large expenditure attributable to training, outreach efforts, grant-in-aid and covering operational expenditure. But, securing sufficient and timely financing for PPM expansion has remained a persistent challenge (Lal, Uplekar, et al. 2011), (Probandari et al. 2011a). The total budget spent under the NGO/PP schemes amounted to Rs 28239385(US\$ 588000) representing just $1.5 \%$ of the total state-level TB expenditure (World Health Organisation 2016). Even these limited funds earmarked for private sector were used for other activities at the state/district level. The fuel expenses for TBHealth Visitors to visit PPs were not covered in the programme and the funds were mostly diverted from the contingency funds available with the District TB Officer. This limited the mobility of field staff to meet PPs periodically. Therefore, it is necessary to ensure the availability of sufficient financial and human resources for setting up PPM schemes (Lal, Uplekar, et al. 2011). Despite being a highly cost effective programme, RNTCP has struggled to receive funding that is commensurate with the scale of India' s epidemic (Pai, Bhaumik, and Bhuyan 2017), (Babu et al. 2016), (Verma 2017).

\section{PATIENT CENTERED CARE}

Recent analyses of the cascade of care in India showed that only about $60 \%$ of the patients were even diagnosed, and only about 40\% accessed RNTCP services (Subbaraman et al. 2016). WHO's post 2015 TB strategy states 'Integrated, patient centered care', as one of the main component of the strategy (World Health organisation 2014). Studies which analysed the reasons for non-adherence to TB treatment in RNTCP have concluded that it stems from a poor match between patients and programmes needs and priorities (Jaiswal et al. 2003), (R. D. Deshmukh et al. 2018). Our study demonstrated that the patients who faced the most difficulty in accessing and completing the DOTS were the most vulnerable to the disease because of their relative level of poverty and their poor social support. This finding resonates the widely recognized phenomenon of the 'Inverse Care Law', which states that those who are in most need of healthcare are least able to access services (Hart 1971).

Our study findings add to the evidence that TB patients in India incur large costs associated with TB illness (Floyd et al. 2006; John et al. 2009; Pantoja, Floyd, et al. 2009b; Tanimura et al. 2014). TB has a devastating socio-economic impact on TB patients and their families and increased their poverty, a finding reported in other studies (Rajeswari et al. 1999), (Mauch et al. 2011). This phenomenon typically illustrates the 'medical poverty trap', a situation where patient's expenditure increases and income decreases (Dahlgren and Whitehead 2006). Government of India has introduced a 
host of patient care initiatives such as nutritional care, and social protection schemes to eliminate catastrophic out of pocket expenditure of patients (Division, Central TB 2017). But several gaps exists in implementing pro-poor strategies in the country (Kamineni et al. 2012).

Our study findings suggest the need to introduce more flexibility in the DOTS provision. Evidence shows that patients who took TB treatment outside RNTCP perceived DOTS in its present form to be rigid and intrusive and compromising privacy (Pinto and Udwadia 2010). Contrastingly, patients in our study who took DOTS from PPs were happy about the flexibility in terms of timing and the timely care provided by PPs when they faced side effects from drugs intake. Women respondents were particularly satisfied about DOTS received from PPs because it provided them privacy, which was often missed at public sector facilities. Yet, we found that field RNTCP staff linked only two patients with PPs for DOTS provision during the study period. The RNTCP has to consider patient centred care as a priority, if its ambitious goal envisaged in the National strategic plan of providing 'universal access to quality assured free diagnosis and treatment to patients', is to become a reality (Sachdeva et al. 2012). The RNTCP also aspires for bold policies such as introduction of a National TB Bill, which will promote TB care as a rights issue and hasten the control of TB in the country. To fulfil this aspiration, the programme has to give due attention to patients' needs, and their expectations from the programme (Chavan 2017), (Pai, Yadav, and Anupindi 2014).

Accumulating evidence has pointed to the effectiveness of a wide variety of approaches such as family-centered DOTS, which produce comparable outcomes to directly observed treatment by healthcare worker (Prado et al. 2011), (Parida et al. 2014). We observed that the adherence to treatment was heavily dependent on patient's and caretaker's competing needs of everyday life. Studies have shown that social support from spouse and close family members was crucial in adhering to the DOTS therapy (Lewis and Newell 2009): the more the social support, the better the coping with the disease condition (Marra et al. 2004). Thus, the TB programme by acknowledging the crucial role of the families should proactively involve them in patient counselling to increase their knowledge and awareness about the disease. It is essential that programme managers recognise that patients strive to adhere to their treatment, but structural barriers prevent them from doing so (Lambert and Stuyft 2005), (Hargreaves et al. 2011), (Farmer 1996). A suggested approach would be one that is responsive to local context, meets patients' expectations and empowers them to complete the treatment (Macq, Torfoss, and Getahun 2007). This needs a change in the mind-set of staff to consider a patient-centered approach, delivered with dignity and compassion.

\section{STRENGTHENING GENERAL HEALTH SYSTEM FOR EFFECTIVE IMPLEMENTATION OF PPM}

Our study provides important information about the systemic impediments that affect engagement of PPs in RNTCP. Most of the published studies have focused on PPM effectiveness (Dewan et al. 2006) and economic analysis (Floyd et al. 2006), without emphasis on the key health system components 
such as resources, service delivery and governance structures that can augment partnerships between public and private health sector. In the following two paragraphs, we discuss health system barriers for PPM implementation.

\subsection{Local Health System Barriers to Engage PPs in RNTCP}

End TB strategy reiterates that it is critical to invest in health system strengthening in order to improve efficiency, effectiveness and accountability of the health system for an improved response to the TB epidemic. India's National Strategic Plan for TB (Central TB Division, Directorate General of Health Services 2017) envisages to strengthen the health system by continuing to develop an integrated approach to TB and leveraging on the existing capacity of the health system to further TB control goals. But explicit operational guidelines on how these could be materialised to align RNTCP strategies and implementation plans with overall health system is critical to realise this vision.

Currently, RNTCP services are organized through a vertical system of health care facilities, both in terms of policy (management and administration) and practice (the operations). There is very little involvement of Medical officers posted at peripheral public health facilities (Primary Health Centres or PHCs), in terms of identifying and developing alliances with potential PPs, sensitising them about PPM-DOTS strategy and monitoring PPM activities. There are arguments indicating that the success of the PPM strategy depends on the capacity of the general health system within which it is delivered (Lei et al. 2015), (Mahendradhata et al. 2007b), (Lönnroth et al. 2004), (Unger, J.P., De Paepe, P., Ghilbert 2010) and how the public sector manages the processes of collaboration (Probandari A, Utarini A, Lindholm L 2011). Studies have reported that the quality of TB care is equally bad in public sector facilities (Jishnu Das, Alaka Holla, Veena Das, Manoj Mohanan, Diana Tabak 2012), (Charles et al. 2010), (Gede et al. 2013), (Rajeswari et al. 2002). Thus, if general health systems does not function well, there are risks that the PPM strategy will also fail to meet the objectives of engaging PPs in the TB care (Atun et al. 2010), (Harries et al. 2009).

Implementation of PPM is a complex phenomenon requiring structural arrangements within the health system. To harmonise RNTCP management and supervision structures within the general health system, efforts have been made by the government to re-organise RNTCP operational units from present TB units to health blocks (1.5 to 2.5 lakh population) of general health system (Central TB Division, Directorate General of Health Services 2017). However, the integration of PPM activities in the health system is yet to happen. Officers at sub-district hardly tend to take any interest in PPM activities and they perceive TB related work to be the sole responsibility of RNTCP staff and district TB officers. A framework which analysed the relationship between disease control programmes and basic health care attempting for integration concluded that the relationship between (vertical) disease control programmes and (horizontal) basic health care services is invariably difficult and advocated for avoiding the dichotomous classification of 'us' and 'them' (Bart, Kegel, and Van 
der Stuyft 2004). Therefore, the best way is to make joint efforts to build competencies that are common to all programmes, for example conducting periodic PP's mapping or implementing PAL, etc (Fairall et al. 2005),(Pio 2005). Also, there are suggestions to link TB programs with those that target diabetes, asthma, chronic obstructive pulmonary disease, anaemia, HIV and/or malaria, and allow PPM to benefit from infrastructure already established by other national health programmes (Bank and Partnership 2014), (World health organisation/CDC/TB/2000.282 2000), (M Uplekar 2016).

\subsection{Health System Regulations and Their Enforcement}

Our study interventions aiming to encourage PPs to notify patients put on private treatment, such as awareness creation about notification, facilitating the collection of notification forms from PP's clinic and constant dialogues with PPs to notify TB patients, were ineffective. Even though India has TB on the national list of notifiable diseases, it does not have the machinery to mandate notification from PPs. A study which mapped the regulatory architecture for health care delivery in India reported that the existence of basic regulations does not automatically imply their adequate enforcement and performance (Sheikh, Saligram, and Prasad 2013). Another study reported that the minimal staffing available for enforcement of mandatory notification in high TB incidence countries has left notification to the goodwill of the and healthcare providers (Mukund Uplekar et al. 2016). Studies trying to understand the barriers to notification in India reported that not all PPs have fully understood the notification's rationale and, although aware, many do not notify. The main reasons being lack of time, concerns regarding patients' confidentiality, lack of trust in the government health system (Philip et al. 2015; Thomas et al. 2016; Yeole et al. 2015). A review of the literature on mandatory TB case-notification policies and practices in high TB-incidence countries (Mukund Uplekar et al. 2016) reported that providers, also in India, preferred to notify through mobile phones, SMS or email (Thomas, Velayutham, and Thiruvengadam 2016). So, it is important for programme managers to look for strategies that will ease the process of reporting from PPs, such as introducing digital tools to simplify notification (Mukund Uplekar et al. 2016).

In India, the policy level has no definitive legislative authority to enforce the bureaucracy and programme managers into implementing the PPM strategy more vigorously. Ensuring notification without legal back up seems to be difficult. However, Uplekar et al in their study caution that regulation may not change people's health seeking behaviour, or help raise provider's index of suspicion for TB, nor will it help in early diagnosis or to ensure the quality of care provided. Thus, it is argued that scaling up PPM will require both collaboration and regulation (Mukund Uplekar 2013). The clinical establishment act of 2010 (Government of India: Ministry of Law and Justice (Legislative Department) 2010) has been enacted by the Central Government to provide for registration and regulation of all clinical establishments in the country with a view to prescribe the minimum standards of facilities and services that could be used as a tool to enforce notification. 
In our study, we took an approach which was primarily based on building a good relationship, strong communication, and persuasion to build the partnership. Considering the absence of a strong regulatory mechanism in the country (Sheikh, Saligram, and Hort 2015), (Bhat 1999), and the limited financial systems available in the programme, we opted for moral persuasion to motivate PPs to involve in the TB care. Some studies have argued that provision of financial incentives to PPs would jeopardise the partnership, and prove unsustainable in the long run and be difficult to replicate (Naser et al. 2012; A. J. Khan et al. 2012). Other studies have shown that apart from financial incentives, PPs identified other incentives, such as access to quality diagnostic services free of charge, free continued education and association with a reputed government programme. These were proven important in initiating and sustaining collaboration (Krishnan et al. 2009), (Lönnroth, Uplekar, and Blanc 2006), (Newell et al. 2004), (Arora, Sarin, and Lönnroth 2003), (Balasubramanian et al. 2006). We thought that once behaviour change was established among PPs and RNTCP staff, it would be self-sustaining, as it would reset the behaviour norms to a better standard of care. Interviews with PPs revealed that the provision of non-financial incentives such as receiving awards from RNTCP by recognising their services for TB care had a greater impact on the PPs. This also gave them a recognition that set them apart from other providers. However, the moral persuasion requires strong advocates at the local level and requires more investments upfront.

However, it must be kept in mind that TB patients are a source of income for PPs and that any partnership model that threatens their income may fail. Our study demonstrated the existence of kickbacks from private labs and pharmacies, and PPs having financial incentives to retain patients (Yellappa, Vijayashree 2014). If financial incentives are opted as a strategy, this will have to be more lucrative than those currently available to PPs by retaining TB patients. Studies have shown that meagre financial incentives had little impact on case treatment and management (Quy, Lönnroth, et al. 2003), (Quy, Lan, et al. 2003). Therefore, as a collaborative measure a combination of enhanced financial incentive and non-financial motivations could improve PP's participation in the provision of TB (Sinanovic and Kumaranayake 2010)

\section{WAY FORWARD}

\subsection{Developing a Supportive Policy and Programmatic Environment}

A successful and sustainable PPP requires a coherent policy-planning framework as well as systematic and proactive follow-up procedures for building a partnership with the private sector. But, PPPs are generally weak in their governance structure and there is desperate need for improvement in this regard (Kent Buse Sonja Tanaka 2011). The planning commission of India, while identifying the barriers for implementing PPPs in health, raised similar concerns of lack of effective governance mechanisms and institutionalised management structures to steer and execute PPM activities (Planning Commsion 2012). 
It is difficult to visualise successful implementation of the PPM strategy in the years to come without greater political and bureaucratic commitment to and clarity on the overall PPM strategy. Strong leadership and good governance exercised by the State play a critical role in creating an enabling environment for private sector actors to contribute to public health, and also to shape the type and quality of contributions that the private sector can make to public goals. Currently, there is no definitive legislative authority to force the bureaucracy and programme managers into implementing the PPM strategy more vigorously and there are no official structures in place in terms of accountability. Therefore, it is important to formulate governance systems and structures in a true spirit of partnership instead of loosely, uncoordinated set of rules that is now often in place.

Earlier PPM studies have reported about the success of engaging NGOs or professional organisations to foster the relation between public and private sector (Rangan et al. 2004), (Ambe, Lönnroth, Dholakia, Copreaux, Zignol, Borremans, Uplekar, et al. 2005), (Lönnroth et al. 2004; Hurtig et al. 2002), (Probandari et al. 2011b). There have been proposals to RNTCP to use Public Private Intermediary Agencies (PPIAs) to engage with PPs in RNTCP (Central TB Division, Directorate General of Health Services 2017), (World Health organisation 2016). There has also been a fair amount of information on PPIAs in countries such as Bangladesh and Cambodia (Wells 2014). Government could an adopt a more strategic approach by employing PPIAs, and step back from the day to day management of PPM activities, but instead prioritise on encouraging alliances and partnerships with the private sector. But a pragmatic question to be answered is whether PPIA's model of human and financial resource-intensive approach is scalable with domestic funding in India. If that is not the case, alternate finance mechanisms need to be explored to achieve this objective and sustaining PPM activities (Wells 2014).

Currently lessons from prior PPM experiments are not available as a repository to share country and partner's experiences, challenges and solutions. As a start, there is an urgent need to build a National knowledge database on PPM experiments, on what works, where and why. There is also a need to include lessons on both successes and failures as part of knowledge building to assemble existing information about working with the private sector. Such reviews should be published on web-based platforms to enable sharing of best practices, resources and ideas, and encourage synergy. For this purpose, a separate PPM secretariat has to be established at CTD, which will oversee the implementation of PPM activities at the National level. The secretariat could take the responsibility of promoting operational research in PPM, asses impact of PPM projects, and guide evidence-based implementation of PPM in the country through regular feedback mechanisms. At provincial level, PPP coordinators could be made responsible for overseeing the PPM in their provinces, and for identifying potential partners. This, however, should start with the development of a separate PPM module to train the public sector to engage with the private sector. This was also recommended by JMM 2015 (World Health Organisation 2016). Components such as training, supervision, monitoring and regular evaluation should be included as the primary elements in PPM modules. Additionally, operational guidelines to build the capacity of frontline health workers of RNTCP and general public 
health services staff to engage with PPs should be part of the such modular training. This would help them in attracting, orienting, support and supervise PPs and make them do their job optimally.

\subsection{Moving from Pilots to Large-Scale Initiatives}

Most PPM experiments reported in India so far are limited to district and/or State level and they remained as pilots, without being considered for replication and scale up. The scale, sustainability, and affordability has remained a major concern. Lack of attention to the issues of sustainability and scale-up during early efforts to test or implement innovation uptake have hampered scale up of PPM initiatives. However, this requires to better understand what works best in a given situation, the circumstances in which they are to be implemented, and whether the model partnership be replicated as whole or only specific components of the partnership. Authors deliberating on the scale up of the health interventions have debated that the focus in the global health field is mostly on achieving high coverage rates of health services, and reducing mortality to the neglect of understanding the processes for how to scale up (Subramanian et al. 2011). While some literature addresses scaling up in the public and private healthcare sectors, much of this work assesses specific disease areas and it is unclear how to generalize the findings (Simmons, Ruth, Peter Fajans 2007). There is greater plurality in the forms of public-private interactions at the country level with unique implementation challenges that required creative solutions to move forward. Therefore, highly varied and contextspecific responses to TB show that solutions need to be documented and compared to develop evidence-based policies and practice (Atun et al. 2010).

With this background, we need to go beyond a definition of what makes a successful pilot to the question of what is important for scaling which provides sustainability. Our model employed multiple intervention strategies at different levels focusing on using and building local RNTCP staff skills to create a replicable model and transfer knowledge to the government for further expansion and scale-up of successful approaches. We hypothesised that a well-designed randomised control trial may alleviate the local contexts, selection bias and confounding inconsequential in the final impact assessment. Thus, provide evidence for causal associations making the intervention generalisable and scaled up by standardisation of successful interventions. In our study we identified successful interventions that could be considered for scaling up were; (i) improved referral and information system using simple, practical tools; (ii) local mapping of all care providers in a systematic manner, and prioritize the providers who are most likely to contribute to TB case notification and treatment; (iii) building the capacity of RNTCP staff to collaborate with PPs; and (iv) a strong commitment on the part of RNTCP to support, supervise and evaluating PPM activities. Nonetheless, replication warrants examination, because of the ability of a program to transplant its model from one context to another context, which typically includes different socio-economic, cultural, and political aspects, that needs to be studied carefully and factored into, while designing the model. 


\subsection{Developing Monitoring and Evaluation Tools for PPM}

Though RNTCP has developed a strong monitoring and supervision system for the public sector performance, a same rigorous PPM-focused monitoring and surveillance system is conspicuously absent for measuring private sector performance. Hence, it is important to standardise and institutionalise the PPM processes and design practical tools that can be used across the region to engage PPs. In the absence of clear performance-related indicators for the PPM, RNTCP staff may circumvent PPM-related activities, as it neither offers any incentives nor contributes to their measurable performance (Engel and van Lente 2014). That is the reason why PPM is viewed as a laborious additional task that does not show any impact on RNTCP programme indicators, cannot be easily measured and thus has no immediate benefit for the health staff in-charge (Lal, Sahu, et al. 2011a). This concern was highlighted by other studies (Aime De Muynck, David Berger, Tim Clary, Selva Kumar 2011) and RNTCP evaluations as well (World Health Organisation 2016). Thus it makes a compelling case to develop a separate PPM reporting system and tools to measure output/outcome indicators to monitor and evaluate the performance of PPM activities (Mukund Uplekar 2003), (Lönnroth et al. 2004). This information should be able to generate a PPM database that can be integrated with public data to produce district-wide PPM reports. This will help the State to objectively assess and regularly review PPM impact and to quantify the extent to which they have contributed to the achievement of national goals. WHO's handbook on how to measure contributions of PPM to TB control can guide the country on setting PPM monitoring and evaluation systems (World Health Organization, Management Sciences for Health 2010), (Organisation, World Health:WHO/HTM/TB/2006.360. Geneva, Switzerland: WHO 2006). Additionally, a supervisory checklist should be developed for the public sector that could be adapted to the characteristics of local PPs in the region.

\section{CONCLUSION}

Our study, conducted in routine programmatic settings, is the first study in India which assessed the participation and contribution of individual for-profit, allopathic PPs in RNTCP. We demonstrated that a system-oriented intervention implemented by RNTCP staff, based on the establishment of a strong referral and communication system with PPs, improved PP's referrals of presumptive pulmonary TB patients to RNTCP. Overall, the intervention arm contributed to $14 \%$ of the bacteriologically confirmed pulmonary TB case detection in the study area. The impact of expansion of the intervention to the control arm, thus covering all eligible PPs, could increase the yield to a quarter or so of all patients referred.

Most studies available are either cross-sectional or longitudinal studies without controls and apparently none with a randomised design. A strength of our study is the randomised controlled trial design, which promises generalisable results. Nonetheless, situated assessment to be able to design interventions that work in specific context was critical in the success of the model. 
Our study, conducted in routine programmatic settings, provides important information about the challenges of planning and implementing PPM by RNTCP staff and the adjustments that should be made in the general health system to improve the efficiency, effectiveness and accountability of PPM strategy. These insights are timely, because of RNTCP's ambitious plan articulated in the National Strategic Plan for TB Elimination 2017-2025 (Central TB Division, Directorate General of Health Services 2017). In order to achieve this objective, the Government must operationalise PPM policy into implementable activities by developing specific guidelines to engage PPs. Furthermore, development of key performance indicators to measure the process and impact of PPM is urgently required. The implementation, and expansion of revised PPM schemes in India is unlikely to be accomplished under the prevailing system of service delivery.

We anticipate that this PPM model should be seen as part of the larger debate on the role of the private health sector and we expect many conclusions to be broadly applicable to the PPP in healthcare. The results and conclusions of this study could be useful for other disease control programmes and interventions for health systems design generally. Based on the outcomes of this study, the intervention is now modified for the rural part of the same district, rolled out and being evaluated for the effectiveness (World Health Organization 2015). 


\section{PART V - REFERENCES}

Achanta, Shanta, Jyoti Jaju, Ajay M V Kumar, Sharath Burugina Nagaraja, Srinivas Rao Motta Shamrao, Sasidhar Kumar Bandi, Ashok Kumar, et al. 2013. "Tuberculosis Management Practices by Private Practitioners in Andhra Pradesh, India.” PloS One 8 (8): e71119. https://doi.org/10.1371/journal. pone.0071119.

Agarwal, S P, L S Chauhan. 2005. Tuberculosis Control in India.

Aime De Muynck, David Berger, Tim Clary, Selva Kumar, Gani Perla. 2011. "Evaluation of Tuberculosis Program in India: WHO Report."

Aleksandrowicz, Lukasz, Varun Malhotra, Rajesh Dikshit, Prakash C. Gupta, Rajesh Kumar, Jay Sheth, Suresh K. Rathi, et al. 2014. "Performance Criteria for Verbal Autopsy-Based Systems to Estimate National Causes of Death: Development and Application to the Indian Million Death Study." BMC Medicine 12 (21): 1-14. https://doi.org/10.1186/1741-7015-12-21.

Ambe, G, K Lönnroth, Y Dholakia, J Copreaux, M Zignol, N Borremans, and M Uplekar. 2005. "Every Provider Counts: Effect of a Comprehensive Public-Private Mix Approach for TB Control in a Large Metropolitan Area in India." The International Journal of Tuberculosis and Lung Disease : The Official Journal of the International Union against Tuberculosis and Lung Disease 9 (5): 562-68.

Ambe, G, K Lönnroth, Y Dholakia, J Copreaux, M Zignol, N Borremans, M Uplekar, Mumbai Municipal Corporation, and World Health Organization. 2005. "Every Provider Counts : Effect of a Comprehensive Public-Private Mix Approach for TB Control in a Large Metropolitan Area in India." Int J Tuberc Lung Dis 9 (5): 562-68.

Anand, Tanu, Ranjith Babu, AnilG Jacob, Karuna Sagili, and SarabjitS Chadha. 2017. "Enhancing the Role of Private Practitioners in Tuberculosis Prevention and Care Activities in India." Lung India 34: 53844. https://doi.org/10.4103/0970-2113.217577.

Annigeri, Vinod B, Prosser Lizann, Jack, and Raghu Roy. 2004. “An Assesent of Public Private Partnership Opportunities in India."

Arfina, Ferdous Osman. n.d. "Public-Private Partnership in Health Service Delivery : Lessons From Bangladesh."

Arinaminpathy, Nimalan, Deepak Batra, Sunil Khaparde, Thongsuanmung Vualnam, Nilesh Maheshwari, Lokesh Sharma, Sreenivas A. Nair, and Puneet Dewan. 2016. "The Number of Privately Treated Tuberculosis Cases in India: An Estimation from Drug Sales Data." The Lancet Infectious Diseases 16 (11): 1255-60. https://doi.org/10.1016/S1473-3099(16)30259-6.

Arora, V K, R Sarin, and K Lönnroth. 2003. "Feasibility and Effectiveness of a Public-Private Mix Project for Improved TB Control in Delhi , India." Int J Tuberc Lung Dis 7 (12): 1131-38.

Atun, Rifat, Diana E C Weil, Mao Tan Eang, and David Mwakyusa. 2010. "Tuberculosis 7 Health-System Strengthening and Tuberculosis Control.” The Lancet 375 (9732): 2169-78. https://doi.org/10.1016/ S0140-6736(10)60493-X.

Ayesha De Costa, Eva Johansson, Vinod K Diwan. 2008. "Barriers of Mistrust: Public and Private Health Sectors' Perceptions of Each Other in Madhya Pradesh, India." Qlalitative Health Research, no. https://doi.org/10.1177/1049732308318504. 
Babu, Ranjith, Karuna D Sagili, Anil G Jacob, and Sarabjit S Chadha. 2016. "Resource Optimisation for Tuberculosis Elimination in India." Economic and Political Weekly II (19): 26-27.

Balasubramanian, R, R Rajeswari, R D Vijayabhaskara, K Jaggarajamma, P G Gopi, V Chandrasekaran, and P R Narayanan. 2006. "A Rural Public-Private Partnership Model in Tuberculosis Control in South India." Int J Tuberc Lung Dis 10 (12): 1380-85.

Baloch, Noor Ahmad, and Madhukar Pai. 2012. "Tuberculosis Control: Business Models for the Private Sector.” The Lancet Infectious Diseases 12 (8): 579-80. https://doi.org/10.1016/S14733099(12)70122-6.

Bank, World, and Stop T B Partnership. 2014. "Public Private Mix ( PPM ) Models for the Sustainability of Successful TB Control Initiatives Table of Contents."

Bart, Criel, Guy Kegel, and P Van der Stuyft. 2004. "A Framework for Analysing the Relationship between Disease Control Programmes and Basic Health Care." Tropical Medicine and International Health 9 (6): A1-4. https://doi.org/10.1111/j.1365-3156.2004.01257.x.

Baru, Rama V, and Madhurima Nundy. 2008. "Blurring of Boundaries: Public-Private Partnerships in Health Services in India." Economic and Political Weekly 43 (4): 62-71. http://www.jstor.org/ stable/40277081.

Basu, Sanjay, Jason Andrews, Sandeep Kishore, Rajesh Panjabi, and David Stuckler. 2012. "Comparative Performance of Private and Public Healthcare Systems in Low- and Middle-Income Countries: A Systematic Review.” PLoS Medicine 9 (6): e1001244. doi:10.1371/journal.pmed.1001244. https://doi. org/10.1371/journal.pmed.1001244.

Bell, C a, G Duncan, and B Saini. 2011. "Knowledge, Attitudes and Practices of Private Sector Providers of Tuberculosis Care: A Scoping Review." The International Journal of Tuberculosis and Lung Disease : The Official Journal of the International Union against Tuberculosis and Lung Disease 15 (8): 100517. https://doi.org/10.5588/ijtld.10.0294.

Bharaswadkar, Sandeep, Avinash Kanchar, Narendra Thakur, Shubhangi Shah, Brinda Patnaik, Eleanor S Click, Ajay M V Kumar, and Puneet Kumar Dewan. 2014. "Tuberculosis Management Practices of Private Practitioners in Pune Municipal Corporation, India." PLoS ONE 9 (6): e97993.

Bhargava, Anurag, Lancelot Pinto, and Madhukar Pai. 2011. "Mismanagement of Tuberculosis in India : Causes , Consequences , and the Way Forward." Hypothesis 9 (1): 1-13.

Bhat, Ramesh. 1999. "Characteristics of Private Medical Practice in India: A Provider Perspective." Health Policy and Planning 14 (1): 26-37. https://doi.org/10.1093/heapol/14.1.26.

Bishwajit, Ghose, Seydou Ide, and Sharmistha Ghosh. 2014. "Social Determinants of Infectious Diseases in South Asia." International Scholarly Research Notices 2014: 1-10. https://doi. org/10.1155/2014/135243.

Boyatzis, Richard E. 1998. "Transforming Qualitative Information: Thematic Analysis and Code Development." Thousand Oaks, CA: Sage.

Burnard, P, P Gill, K Stewart, E Treasure, and B Chadwick. 2008. "Analysing and Presenting Qualitative Data.” British Dental Journal 204 (8): 429-32. https://doi.org/10.1038/sj.bdj.2008.292.

Caminero, A, and Nils E Billo. 2003. "Involving Private Practitioners and Chest Physicians in the Control of Tuberculosis" 9792: 148-55. https://doi.org/10.1016/S1472-9792(02)00055-0.

Cazabon, Danielle, Hannah Alsdurf, Srinath Satyanarayana, Ruvandhi Nathavitharana, Ramnath Subbaraman, Amrita Daftary, and Madhukar Pai. 2017. "Quality of Tuberculosis Care in High Burden Countries: The Urgent Need to Address Gaps in the Care Cascade." International Journal of Infectious Diseases 56: 111-16. https://doi.org/10.1016/j.ijid.2016.10.016. 
Central TB Division, Directorate general of health services, Ministry of health and family welfare. 2014. "National Guidelines for Partnership 2014."

Central TB Division, Directorate General of Health Services, Ministry of Health with Family Welfare. 2017. "National Strategic Plan for Tuberculosis Elimination 2017-2025." https://tbcindia.gov.in/ WriteReadData/NSP Draft 20.02.2017 1.pdf.

central TB Division,Directorate general of health services, Ministry of health and family welfare. 2001. PPM Schemes for PPs.

. 2012. "National Strategic Plan for Tuberculosis Control 2012-2017." https://www.tbfacts.org/wpcontent/uploads/2016/01/NSP-2012-2017.pdf.

Central TB Division; Government of India; 2017. “TB India 2017.” RNTCP Annual Status Report https:// tb: 1-173. http://www.tbcindia.nic.in/WriteReadData/TB India 2017.pdf.

Central TB Division; Government of India. 2016. "Revised National TB Control Programme: Tecnical and Operational Guidelines for Tuberculosis Control in India-Chapter 8-Recording and Reporting." Ministry of Health and Family Welfare, Government of India.

Central TB Division. 2008. Revised Schemes for NGOs and Private Providers.

Central TB Division, Ministry of Health and Family Welfare. 2018. "India TB Report 2018: Revised Natonal TB Control Programme, Anual Status Report."

Charles, Niruparani, Beena Thomas, Basilea Watson, M Raja Sakthivel, V Chandrasekeran, and Fraser Wares. 2010. "Care Seeking Behavior of Chest Symptomatics: A Community Based Study Done in South India after the Implementation of the RNTCP." PloS One 5 (9): 1-6. https://doi.org/10.1371/ journal.pone. 0012379 .

Chavan, Deepti. 2017. "Fighting TB Requires Empowered Patients.” BMJ 356: 1-2. https://doi. org/10.1136/bmj.i6344.

Costa, A., Johansson, E., \& Diwan, V. K. (2008). De. 2008. "Barriers of Mistrust: Public and Private Health Sectors' Perceptions of Each Other in Madhya Pradesh, India. Qualitative.” Qualitative Health Research 18: 756-766.

Costa, A De, T Kazmi, K Lönnroth, M Uplekar, V K Diwan, Ruxmaniben Deepchand, Gardi Medical, and Karolinska Institutet. 2008. "SHORT COMMUNICATION PPM : ' Public-Private ' or ' PrivatePublic 'Mix ? The Case of Ujjain District, India.” Int J Tuberc Lung Dis 12 (11): 1333-35.

Cross, Jamie, and Hayley Macgregor. 2009. "Working Paper: Who Are 'Informal Health Providers ' and What Do They Do ? Perspectives from Medical Anthropology.” Vol. 334.

Dahlgren, Goran, and Margaret Whitehead. 2006. "European Strategies for Tackling Social Inequities in Health: Levelling up Part 2." World Health Organization, no. 3: 1-140.

Das, Jishnu, Ada Kwan, Benjamin Daniels, Srinath Satyanarayana, Ramnath Subbaraman, Sofi Bergkvist, Ranendra K Das, Veena Das, and Madhukar Pai. 2015. "Use of Standardised Patients to Assess Quality of Tuberculosis Care : A Pilot , Cross-Sectional Study." The Lancet Infectious Diseases 3099 (15). https://doi.org/10.1016/S1473-3099(15)00077-8.

Deshmukh, R. D., D. J. Dhande, K. S. Sachdeva, A. N. Sreenivas, A. M.V. Kumar, and M. Parmar. 2018. "Social Support a Key Factor for Adherence to Multidrug-Resistant Tuberculosis Treatment." Indian Journal of Tuberculosis 65 (1): 41-47. https://doi.org/10.1016/j.ijtb.2017.05.003.

Deshmukh, Rajesh D, D J Dhande, and Kuldeep Singh Sachdeva. 2015. "Patient and Provider Reported Reasons for Lost to Follow Up in MDRTB Treatment : A Qualitative Study from a Drug Resistant TB Centre in India." PLoS ONE 10 (8): 1-11. https://doi.org/10.1371/journal.pone.0135802.

Dewan, Puneet K, S S Lal, Knut Lonnroth, Fraser Wares, Mukund Uplekar, Suvanand Sahu, Reuben Granich, and Lakhbir Singh Chauhan. 2006. "Improving Tuberculosis Control through Public- 
Private Collaboration in India: Literature Review." BMJ 332 (7541): 574-78. https://doi.org/10.1136/ bmj.38738.473252.7C.

DirecHealth and Family Welfare, Government of India, central TB Division. 2012. Guidance for TB Notification in India.

Directorate General of Health Services Ministry of Health \& Family Welfare, and Government of India. 2012. "Indian Public Health Standards (IPHS) Guidelines for Primary Health Centres Revised 2012." Indian Public Health Standards (IPHS), 1-100. http://nhm.gov.in/images/pdf/guidelines/iphs/iphsrevised-guidlines-2012/primay-health-centres.pdf.

Division, Central T B,directorate general of health services, Ministry of health and family welfare. 2000. "Involvement of NGOs in RNTCP."

Division, Central TB, Ministry of health and family welfare. 2017. "Guidance Document: Nutritional Care and Support for Patients with Tuberculosis in India." http://www.tbcindia.nic.in/WriteReadData/ Guidance Document - Nutritional Care \%26 Support for TB patients in India.pdf.

Eisenhardt, Kathleen M., and Melissa E. Graebner. 2007. "Theory Building from Cases: Opportunities and Challenges." Academy of Management Journal 50 (1): 25-32. https://doi.org/10.5465/ AMJ.2007.24160888.

Engel, Nora, and Wiebe Bijker. 2012. "Innovating Tuberculosis Control in India." Economic and Political Weekly XLVII (4): 111-18.

Engel, Nora, and H van Lente. 2014. "Organisational Innovation and Control Practices: The Case of Public-Private Mix in Tuberculosis Control in India." Sociology of Health and Illness Organizational 36 (6): 917-31.

Engel, Nora, and Harro van Lente. 2013. "Organisational Innovation and Control Practices: The Case of Public-Private Mix in Tuberculosis Control in India." Sociology of Health and Illness 36 (6): 917-31. https://doi.org/10.1111/1467-9566.12125.

Fairall, Lara R, Merrick Zwarenstein, Eric D Bateman, Max Bachmann, Carl Lombard, Bosielo P Majara, Gina Joubert, et al. 2005. "Effect of Educational Outreach to Nurses on Tuberculosis Case Detection and Primary Care of Respiratory Illness: Pragmatic Cluster Randomised Controlled Trial." BMJ (Clinical Research Ed.) 331: 750-54. https://doi.org/10.1136/bmj.331.7519.750.

Farmer, Paul. 1996. "Social Inequalities and Emerging Infectious Diseases." Emerging Infectious Diseases 2 (4): 259-69.

Ferroussier, O, M K A Kumar, P K Dewan, P K J Nair, S Sahu, D F Wares, K Laserson, C Wells, R Granich, and L S Chauhan. 2007. "Cost and Cost-Effectiveness of a Public-Private Mix Project in Kannur District, Kerala , India , 2001 - 2002.” Int J Tuberc Lung Dis 11 (7): 755-61.

Floyd, Katherine, V K Arora, K J R Murthy, Knut Lonnroth, Neeta Singla, Y Akbar, and Matteo Zignol. 2006. "Cost and Cost-Effectiveness of PPM-DOTS for Tuberculosis Control : Evidence from India." Bulletin of the World Health Organization 84 (6): 437-45.

Gede, I Wayan, Artawan Eka, Ni Wayan, Arya Utami, I Ketut Suarjana, I Made Kerta Duana, Cok Istri, et al. 2013. "Factors Associated to Referral of Tuberculosis Suspects by Private Practitioners to Community Health Centres in Bali Province, Indonesia." BMC Health Services Research 13 (1): 1. https://doi.org/10.1186/1472-6963-13-445.

Geeta Pardeshi, Andrea Deluca, Sutapa Agarwal, Jugal Kishore. 2018. "Tuberculosis Patients Not Covered by Treatment in Public Health Services: Findings from India's National Family Health Survey 201516." Trop Med Int Health, no. https://doi.org/10.1111/tmi.13086.

Government of India: Ministry of Law and Justice (Legislative Department). 2010. Clinical Establishments (Registration and Regulation) Act 2010. Nr23 of 2010. New Delhi. 
Government of India. 2012. "Draft Report on Recommendation of Task Force on Public Private Partnership for the 11th Plan."

Government of India, Ministry of Health \& Family Welfare. 2015. Notification of TB Cases.

Government of India, Planning Commission. 2004. "Public Private Partnership: Report of the PPP SubGroup on Social Sector." http://planningcommission.nic.in/reports/genrep/rep_ppp.pdf.

Granich, R, and L S Chauhan. 2005. "Improved Tuberculosis Case Detection through Public-Private Partnership and Laboratory-Based Surveillance , Kannur District , Kerala , India , 2001 - 2002." Int J TB Lung 9 (December 2004): 870-76.

Harding, April. 2009. "Partnerships with the Private Sector in Health: What the International Community Can Do to Strengthen Health Systems in Developing Countries." Center for Global Development https://ww (November): 3. http://www.cgdev.org/files/1423350_file_CGD_PSAF_Report_web.pdf.

Hargreaves, James R, Delia Boccia, Carlton a Evans, Michelle Adato, Mark Petticrew, and John D H Porter. 2011. "The Social Determinants of Tuberculosis: From Evidence to Action." American Journal of Public Health 101 (4): 654-62. https://doi.org/10.2105/AJPH.2010.199505.

Harries, a D, P M Jensen, R Zachariah, I D Rusen, and D a Enarson. 2009. "How Health Systems in SubSaharan Africa Can Benefit from Tuberculosis and Other Infectious Disease Programmes." The International Journal of Tuberculosis and Lung Disease : The Official Journal of the International Union against Tuberculosis and Lung Disease 13 (10): 1194-99.

Hart, Julian Tudor. 1971. "The Inverse Care Law.” Lancet, no. February,27.

Hazarika, Indrajit. 2011. "Role of Private Sector in Providing Tuberculosis Care: Evidence from a Population-Based Survey in India." Journal of Global Infectious Diseases 3 (1): 19-24. https://doi. org/10.4103/0974-777X.77291.

Herbert J. Rubin \& Irene S. Rubin. 2005. Qualitative Interviewing: The Art of Hearing Data.

Hurtig, A K, S B Pande, S C Baral, J Newell, J D H Porter, and D S Bam. 2002. "Linking Private and Public Sectors in Tuberculosis Treatment in Kathmandu Valley, Nepal.” Health Policy and Planning 17 (1): 78-89.

Indian Council of Medical Research, Public Health Foundation of India, Institute for Health Metrics and Evalaution. 2017. "Health of the Nation's States- The India State-Level Disease Burden Initiative." Department of Health Research, MoHFW. file://Users/vijaya/Desktop/India_Health_of_the_ Nation's_States_Report_2017.pdf.

Isaakidis, Petros, Sheela Rangan, Anagha Pradhan, Joanna Ladomirska, Tony Reid, and Karina Kielmann. 2013. "“ I Cry Every Day ': Experiences of Patients Co-Infected with HIV and Multidrug-Resistant Tuberculosis.” Tropical Medicine \& International Health 00 (00): 1-6. https://doi.org/10.1111/ tmi.12146.

Jaggarajamma, K, R Balambal, M Muniyandi, M Vasantha, Beena Thomas, C Nirupa, G Sudha, V Chandrasekaran, and Fraser Wares. 2009. "PERCEPTIONS OF TUBERCULOSIS PATIENTS ABOUT PRIVATE PROVIDERS BEFORE AND AFTER IMPLEMENTATION OF REVISED NATIONAL," 185-90.

Jaiswal, A, V Singh, J A Ogden, J D H Porter, P P Sharma, R Sarin, V K Arora, and R C Jain. 2003. "Adherence to Tuberculosis Treatment : Lessons from the Urban Setting of Delhi , India." Tropical Medicine \& International Health 8 (7): 625-33.

Jarosawlski S, Pai M. 2012. "Why TB Is Inaccurately Diagnosed in India- A Root Cause Analysis." $J$ Epidemiol Glob Health 2: 39-50.

Jens K. Roehrich, Michael A. Lewis, Gerard George. 2014. "Are Public-Private Partnerships a Healthy Option? A Systematic Literature Review." Social Science and Medicine 113: 110-19. https://doi. 
org/10.1016/j.socscimed.2014.03.037.

Jill Schofield. 2004. "A Model of Learned Implementation.” Public Administration 82 (2).

Jishnu Das, Alaka Holla, Veena Das, Manoj Mohanan, Diana Tabak, Brian Chan. 2012. "In Urban And Rural India, A Standardized Patient Study Showed Low Levels Of Provider Training And Huge Quality Gaps." Global Health 31 (12): 2774-2784 10.1377/hlthaff.2011.1356.

John, K R, P Daley, N Kincler, O Oxlade, and D Menzies. 2009. "Costs Incurred by Patients with Pulmonary Tuberculosis in Rural India." Int J Tuberc Lung Dis 13 (10): 1281-87.

Kamineni, Vishnu Vardhan, Nevin Wilson, Anand Das, Srinath Satyanarayana, and Sarabjit Chadha. 2012. "Addressing Poverty through Disease Control Programmes : Examples from Tuberculosis Control in India." International Journal for Equity in Health 11 (1): 17. https://doi.org/10.1186/1475-9276-1117.

Kapoor, Sunil K, A Venkat Raman, Kuldeep Singh Sachdeva, and Srinath Satyanarayana. 2012. "How Did the TB Patients Reach DOTS Services in Delhi? A Study of Patient Treatment Seeking Behavior." PloS One 7 (8): e42458. https://doi.org/10.1371/journal.pone.0042458.

Kent Buse Sonja Tanaka. 2011. "Global Public $\square$ Private Health Partnerships: Lessons Learned from Ten Years of Experience and Evaluation.” International Dental Journal 61 (s2): doi.org/10.1111/j.1875595X.2011.00034.x.

Khan, Aamir J, Saira Khowaja Msw, Faisal S Khan, Fahad Qazi Mph, Ismat Lotia Mph, Ali Habib Meng, Shama Mohammed, et al. 2012. "Engaging the Private Sector to Increase Tuberculosis Case Detection : An Impact Evaluation Study.” The Lancet Infectious Diseases 12 (8): 608-16. https://doi. org/10.1016/S1473-3099(12)70116-0.

Khan, Mishal S, Solomon Salve, and John D H Porter. 2015. "Engaging For-Profit Providers in TB Control : Lessons Learnt from Initiatives in South Asia." Health Policy Abd Planning 30 (doi:10.1093/heapol/czu137): 1289-95. https://doi.org/10.1093/heapol/czu137.

Kielmann, Karina, Vinita Datye, and Anagha Pradhan. 2014. "Global Public Health : An International Journal for Research, Policy and Practice Balancing Authority, Deference and Trust across the Public - Private Divide in Health Care : Tuberculosis Health Visitors in Western Maharashtra , India." Global Public Health 9 (8): 975-92. https://doi.org/10.1080/17441692.2014.941898.

Kielmann, Karina, Vinita Datye, Anagha Pradhan, and Sheela Rangan. 2014. "Balancing Authority, Deference and Trust across the Public-Private Divide in Health Care: Tuberculosis Health Visitors in Western Maharashtra, India." Global Public Health 9 (8): 975-92. https://doi.org/10.1080/17441692.2 014.941898 .

Kilbourne, Amy M, Mary S Neumann, Harold a Pincus, Mark S Bauer, and Ronald Stall. 2007. "Implementing Evidence-Based Interventions in Health Care: Application of the Replicating Effective Programs Framework." Implementation Science : IS 2 (January): 42. https://doi.org/10.1186/17485908-2-42.

Krishnan, N, R Ananthakrishnan, S Augustine, N K Vijayalakshmi, P G Gopi, V Kumaraswami, and P R Narayanan. 2009. "Impact of Advocacy on the Tuberculosis Management Practices of Private Practitioners in Chennai City , India." Int J Tuberc Lung Dis 13 (1): 112-18.

Kulkarni, V, A Bajpai, C Palanivel, B Unnikrrishnan, T Kanchan, Mohan Kp, N Kumar, R Holla, and R Thapar. 2014. "Awareness and Practices of Private Medical Practitioners Regarding Pulmonary TB in Mangalore, India.” Medical Science 7 (23): 8-14.

Kumar, M. K A, Puneet K. Dewan, P. K J Nair, T. R. Frieden, S. Sahu, F. Wares, K. Laserson, C. Wells, R. Granich, and L. S. Chauhan. 2005. "Improved Tuberculosis Case Detection through PublicPrivate Partnership and Laboratory-Based Surveillance, Kannur District, Kerala, India, 2001-2002.” International Journal of Tuberculosis and Lung Disease 9 (8): 870-76. 
Lal, S. S., S. Sahu, F. Wares, K. Lönnroth, L. S. Chauhan, and M. Uplekar. 2011a. "Intensified Scale-up of Public-Private Mix: A Systems Approach to Tuberculosis Care and Control in India." International Journal of Tuberculosis and Lung Disease 15 (1): 97-104.

Lal, S S, S Sahu, F Wares, K Lönnroth, L S Chauhan, and M Uplekar. 2011b. "Intensifi Ed Scale-up of Public-Private Mix : A Systems Approach to Tuberculosis Care and Control in India." Int J Tuberc Lung Dis 15 (1): 97-104.

Lal, S S, Mukund Uplekar, Itamar Katz, Knut Lonnroth, Ryuichi Komatsu, Hannah Monica, and Yesudian Dias. 2011. "Global Fund Financing of Public - Private Mix Approaches for Delivery of Tuberculosis Care." Tropical Medicine \& International Health 16 (6): 685-92. https://doi.org/10.1111/j.13653156.2011.02749.x.

Lambert, M L, and Patrick Van Der Stuyft. 2005. "Editorial : Delays to Tuberculosis Treatment : Shall We Continue to Blame the Victim ?" Tropical Medicine \& International Health 10 (10): 945-46. https:// doi.org/10.1111/j.1365-3156.2005.01485.x.

Law, Stephanie, Amy S Piatek, Cheri Vincent, Olivia Oxlade, and Dick Menzies. 2017. "Emergence of Drug Resistance in Patients with Tuberculosis Cared for by the Indian Health-Care System : A Dynamic Modelling Study." The Lancet Public Health 2 (1): e47-55. https://doi.org/10.1016/S24682667(16)30035-4.

Lei, Xun, Qin Liu, Erin Escobar, Johane Philogene, Hang Zhu, Yang Wang, and Shenglan Tang. 2015. "Public-Private Mix for Tuberculosis Care and Control: A Systematic Review." International Journal of Infectious Diseases 34: 20-32. https://doi.org/10.1016/j.ijid.2015.02.015.

Lewis, Christopher P, and James N Newell. 2009. "Improving Tuberculosis Care in Low Income Countries - a Qualitative Study of Patients' Understanding of 'Patient Support' in Nepal." BMC Public Health 9: 190. https://doi.org/10.1186/1471-2458-9-190.

Lönnroth, Knut, Mukund Uplekar, Vijay K Arora, Sanjay Juvekar, Nguyen T N Lan, and David Mwaniki. 2004. "Public - Private Mix for DOTS Implementation : What Makes It Work?" Bulletin of the World Health Organization 82 (8).

Lönnroth, Knut, Mukund Uplekar, and Léopold Blanc. 2006. "Hard Gains through Soft Contracts : Productive Engagement of Private Providers in Tuberculosis Control." Bulletin of the World Health Organization 84 (11).

Macq, Jean, Ted Torfoss, and Haileyesus Getahun. 2007. "Patient Empowerment in Tuberculosis Control : Reflecting on Past Documented Experiences.” Tropical Medicine \& International Health 12 (7): 87385. https://doi.org/10.1111/j.1365-3156.2007.01858.x.

Mahendradhata, Yodi, Marie-laurence Lambert, Marleen Boelaert, and Patrick Van Der Stuyft. 2007a. "Editorial : Engaging the Private Sector for Tuberculosis Control : Much Advocacy on a Meagre Evidence Base." Tropical Medicine \& International Health 12 (3): 315-16.

. 2007b. "Editorial : Engaging the Private Sector for Tuberculosis Control : Much Advocacy on a Meagre Evidence Base.” Tropical Medicine \& International Health 12 (3): 315-16.

Mahendradhata, Yodi, Marie-laurence Lambert, Armand Van Deun, Francine Matthys, Marleen Boelaert, and Patrick Van Der Stuyft. 2003. "Strong General Health Care Systems : A Prerequisite to Reach Global Tuberculosis Control Targets Y." International Journal of Health Planning and Management 18: 53-65.

Marra, Carlo A, Fawziah Marra, Victoria C Cox, Anita Palepu, and Mark Fitzgerald. 2004. "Factors Influencing Quality of Life in Patients with Active Tuberculosis." Health and Quality of Life Outcomes 2 (58): doi:10.1186/1477-7525-2-58. https://doi.org/10.1186/1477-7525-2-58.

Mauch, Verena, Naomi Woods, Beatrice Kirubi, Hillary Kipruto, Joseph Sitienei, and Eveline Klinkenberg. 2011. "Assessing Access Barriers to Tuberculosis Care with the Tool to Estimate Patients ' 
Costs : Pilot Results from Two Districts in Kenya." BMC Public Health 11 (1): 43. https://doi. org/10.1186/1471-2458-11-43.

Maung, M, H Kluge, T Aye, W Maung, P Noe, M Zaw, S P Jost, M Uplekar, and K Lönnroth. 2006. "Private GPs Contribute to TB Control in Myanmar : Evaluation of a PPM Initiative in Mandalay Division." Int J Tuberc Lung Dis 10 (9): 982-87.

May, Christina, Katja Roth, and Pradeep Panda. 2014. "Non-Degree Allopathic Practitioners as First Contact Points for Acute Illness Episodes : Insights from a Qualitative Study in Rural Northern India" 14 (1): 1-11. https://doi.org/10.1186/1472-6963-14-182.

McDowell, A., and M. Pai. 2016. "Treatment as Diagnosis and Diagnosis as Treatment: Empirical Management of Presumptive Tuberculosis in India." International Journal of Tuberculosis and Lung Disease 20 (4): 536-43. https://doi.org/10.5588/ijtld.15.0562.

McPake, Barbara, and Anne Mills. 2000. "What Can We Learn from International Comparisons of Health Systems and Health System Reform?" Bulletin of the World Health Organization 78 (6): 811-20. http://eresearch.qmu.ac.uk/326/.

Mills, Anne, Ruairi Brugha, Kara Hanson, and Barbara Mcpake. 2002. "What Can Be Done about the Private Health Sector in Low-Income Countries?" Bulletin of the World Health Organization 80 (4): $325-30$.

Minary, Laetitia, François Alla, Linda Cambon, Joelle Kivits, and Louise Potvin. 2018. "Addressing Complexity in Population Health Intervention Research: The Context/Intervention Interface.” Journal of Epidemiology and Community Health 72: 319-323. doi:10.1136/jech-2017-209921. https://doi. org/10.1136/jech-2017-209921.

Mishra, Gyanshankar, and Jasmin Mulani. 2013. "Tuberculosis Prescription Practices In Private And Public Sector In India." Natl J Integr Res Med 4 (2): 71-78.

Mistry, Nerges, Eunice Lobo, Shimoni Shah, Sheela Rangan, and Yatin Dholakia. 2017. "Pulmonary Tuberculosis in Patna, India: Durations, Delays, and Health Care Seeking Behaviour among Patients Identified through Household Surveys." Journal of Epidemiology and Global Health 7 (4): 241248 ;http://dx.doi.org/10.1016/j.jegh.2017.08. https://doi.org/10.1016/j.jegh.2017.08.001.

MQ, Patton. 2002. "Qualitative Research and Evaluation Methods." 3rd Edition. Thousand Oaks, CA: Sage.

Muraleedharan, Vangal R, Sonia Andrews, Bhuvaneswari Rajaraman, and Stephen Jan. 2005. "Private Sector in the Revised National Tuberculosis Control Programme : A Study of the Implementation of Private-Public Partnership Strategy in Tamil Nadu and Kerala." Health Economics and Financing Programme.

Murthy, K J R, T R Frieden, A Yazdani, and P Hreshikesh. 2001. "Public-Private Partnership in Tuberculosis Control : Experience in Hyderabad, India" 5 (June 2000): 354-59.

Naser, Abu, Zafar Ullah, Rumana Huque, Ashaque Husain, Salma Akter, Akramul Islam, and James Nicholas Newell. 2012. "Effectiveness of Involving the Private Medical Sector in the National TB Control Programme in Bangladesh : Evidence from Mixed Methods." https://doi.org/10.1136/ bmjopen-2012-001534.

Neeraj Kulshrestha a, Sreenivas Achuthan Nair b, K. Rade c, A. Moitra c, P. Diwand, S.D. Khaparde. 2015. "Public-Private Mix for TB Care in India: Concept, Evolution, Progress." Indian Journal of Tuberculosis 62: 235-38.

Newell, James N, Shanta B Pande, Sushil C Baral, Dirgh S Bam, Pushpa Malla, and James N Newell. 2004. "Control of Tuberculosis in an Urban Setting in Nepal : Public - Private Partnership." Bulletin of the World Health Organization 82 (2). 
Nimbarte, Sanjay B, Vasant Wagh, and Deepak Selokar. 2011. "Health Seeking Behaviour among Pulmonary Tuberculosis Patients in Rural Part of Central India." International Journal of Biological and Medical Research 2 (1): 394-97.

Nishtar, Sania. 2007. "Politics of Health Systems: WHO’s New Frontier." Lancet 370 (9591): 935-36. https://doi.org/10.1016/S0140-6736(07)61442-1.

Nora Engel, Harro van Lente. 2013. "Organizational Innovation and Control Practices: The Case of PublicPrivate Mix in Tuberculosis Control in India." Sociology of Health and Illness 36 (6): 917-31.

Nwe, Thin Thin, Saw Saw, Le Win, Myo Myo Mon, Johan van Griensven, Shuisen Zhou, Palanivel Chinnakali, Safieh Shah, Saw Thein, and Si Thu Aung. 2017. "Engagement of Public and Private Medical Facilities in Tuberculosis Care in Myanmar: Contributions and Trends over an Eight-Year Period." Infectious Diseases of Poverty 6 (1): 1-7. https://doi.org/10.1186/s40249-017-0337-8.

World Health Organisation:WHO/HTM/TB/2006.360. Geneva, Switzerland: WHO, 2006. 2006. "Engaging All Health Care Providers in TB Control: Guidance on Implementing Public-Private Mix Approaches."

World Health Organisation. 2014. "Standards for TB Care in India." - 2017. "Executive Summary."

Pai, Madhukar, Soumyadeep Bhaumik, and Soumitra S Bhuyan. 2017. "India's Plan to Eliminate Tuberculosis by 2025 : Converting Rhetoric into Reality." BMJ Global Health 2 (e000326. doi:10.1136/bmjgh-2017-000326): 15-16. https://doi.org/10.1136/bmjgh-2017-000326.

Pai, Madhukar, Prashant Yadav, and Ravi Anupindi. 2014. "Tuberculosis Control Needs a Complete and Patient-Centric Solution.” The Lancet Global Health 2 (4): e189-90. https://doi.org/10.1016/S2214109X(14)70198-6.

Pantoja, A, K Floyd, K P Unnikrishnan, R Jitendra, M R Padma, S S Lal, M Uplekar, et al. 2009a. "Economic Evaluation of Public-Private Mix for Tuberculosis Care and Control, India . Part I . Socio-Economic Profi Le and Costs among Tuberculosis Patients." Int J Tuberc Lung Dis 13 (6): 698-704.

2009b. "Economic Evaluation of Public-Private Mix for Tuberculosis Care and Control, India . Part I . Socio-Economic Profi Le and Costs among Tuberculosis Patients." Int J TB Lung 13 (December 2008): 698-704.

Pantoja, A, K Lönnroth, S S Lal, L S Chauhan, M Uplekar, M R Padma, K P Unnikrishnan, et al. 2009. "Economic Evaluation of Public-Private Mix for Tuberculosis Care and Control, India . Part II . Cost and Cost-Effectiveness." Int J Tuberc Lung Dis 13 (6): 705-12.

Parida, Amrita, K L Bairy, Bharti Chogtu, Rahul Magazine, and Sudha Vidyasagar. 2014. "Comparison of Directly Observed Treatment Short Course ( DOTS ) with Self-Administered Therapy in Pulmonary Tuberculosis in Udupi District of Southern India." Journal of Clinical and Diagnostic Research 8 (8): 29-31. https://doi.org/10.7860/JCDR/2014/8865.4721.

Pethani, Amin, Mubashir Zafar, Adeel Ahmed Khan, Unaib Rabbani Sana, Sana Ahmed, and Zafar Fatmi. 2015. "Engaging General Practitioners in Public-Private Mix Tuberculosis DOTS Program in an Urban Area in Pakistan: Need for Context-Specific Approach." Asia-Pacific Journal of Public Health 27 (2): NP984-92.

Philip, Sairu, Petros Isaakidis, Karuna D. Sagili, Asanarupillai Meharunnisa, Sunilkumar Mrithyunjayan, and Ajay M. V. Kumar. 2015. ““'They Know, They Agree, but They Don't Do”- The Paradox of Tuberculosis Case Notification by Private Practitioners in Alappuzha District, Kerala, India." Plos One 10 (4): e0123286. https://doi.org/10.1371/journal.pone.0123286. 
Pinto, Lancelot M, and Zarir F Udwadia. 2010. "Private Patient Perceptions about a Public Programme ; What Do Private Indian Tuberculosis Patients Really Feel about Directly Observed Treatment ?" BMC Public Health 10 (357): 1471-2458.

Pio, Antonio. 2005. "Practical Approach to Lung Health ( PAL ) A Primary Health Care Strategy." World Health Organization. Vol. WHO/HTM/TB.

Planning Commsion, Government of India. 2012. "Draft Report on Recommendation of Task Force on Public Private Partnership."

Pradhan, Anagha, Vinita Datye, Karina Kielmann, Kishor Khilare, Amruta Datye, Vikas Inamdar, John Porter, and Sheela Rangan. 2011a. "SUSTAINING PPM-DOTS : THE CASE OF PIMPRI CHINCHWAD , MAHARASHTRA, INDIA.” Indian Journal of Tuberculosis 58: 18-28. . 2011b. "SUSTAINING PPM-DOTS : THE CASE OF PIMPRI CHINCHWAD, MAHARASHTRA, INDIA.” Indian Journal Tuberculosis 58: 18-28.

Prado, T N, N Wada, L M Guidoni, J E Golub, R Dietze, and E L Maciel. 2011. "Cost-Effectiveness of Community Health Worker versus Home-Based Guardians for Directly Observed Treatment of Tuberculosis in Vitoria, Espirito Santo State, Brazil." Cadernos De Saude Publica 27 (5): 944-52. https://doi.org/S0102-311X2011000500012 [pii].

Probandari A, Utarini A, Lindholm L, Hurtig A: 2011. "Life of a Partnership: The Process of Collaboration between the National Tuberculosis Program and the Hospitals in Yogyakarta, Indonesia." Soc Sci Med 73 (9): 1386-1394.

Probandari, Ari, Adi Utarini, Lars Lindholm, and Anna-karin Hurtig. 2011a. "Social Science \& Medicine Life of a Partnership : The Process of Collaboration between the National Tuberculosis Program and the Hospitals in Yogyakarta , Indonesia." Social Science \& Medicine 73 (9): 1386-94. https://doi. org/10.1016/j.socscimed.2011.08.017.

. 2011b. "Social Science \& Medicine Life of a Partnership : The Process of Collaboration between the National Tuberculosis Program and the Hospitals in Yogyakarta , Indonesia." Social Science \& Medicine 73 (doi:10.1016/j.socscimed.2011.08.017): 1386-94. https://doi.org/10.1016/j. socscimed.2011.08.017.

PwC Health Research Institution. 2010. "Build and Beyond:The (r)Evolution of Healthcare PPPs." PricewaterhouseCoopers.

Quelin, Ilze Kivleniece and Bertrand V. 2012. "Creating and Capturing Value in Public-Private Ties: A Private Actor's Perspective." Academy of Management 37 (2): https://doi.org/10.5465/amr.2011.0004.

Quy, H T, N T N Lan, K Lönnroth, T N Buu, T T N Dieu, and L T Hai. 2003. "Public-Private Mix for Improved TB Control in Ho Chi Minh City, Vietnam : An Assessment of Its Impact on Case Detection." Int J Tuberc Lung Dis 7 (5): 464-71.

Quy, H T, K Lönnroth, N T N Lan, and T N Buu. 2003. "Treatment Results among Tuberculosis Patients Treated by Private Lung Specialists Involved in a Public-Private Mix Project in Vietnam." Int $J$ Tuberc Lung Dis 7 (12): 1139-46.

R.Reich, IlavenilRamiahMichael. 2006. "Building Effective Public-Private Partnerships: Experiences and Lessons from the African Comprehensive HIV/AIDS Partnerships (ACHAP)." Siocial Science and Medicine 63 (2): 397-408; https://doi.org/10.1016/j.socscimed.2006.

Rajeswari, R, R Balasubramanian, M Muniyandi, S Geetharamani, X Thresa, and P Venkatesan. 1999. "Socio-Economic Impact of Tuberculosis on Patients and Family in India." Int J Tuberc Lung Dis 3 (10): 869-77.

Rajeswari, R, V Chandrasekaran, M Suhadev, S Sivasubramaniam, G Sudha, and G Renu. 2002. "Factors Associated with Patient and Health System Delays in the Diagnosis of Tuberculosis in South India." International Journal of TB and Lung Dieases 6 (9): 789-95. 
Rangan, S G, S K Juvekar, S B Rasalpurkar, S N Morankar, A N Joshi, and J D H Porter. 2004.

"Tuberculosis Control in Rural India : Lessons from Public-Private Collaboration SUMMARY." Int J Tuberc Lung Dis 8 (5): 552-59.

Reid, Michael J.A., and Eric Goosby. 2017. "Patient-Centered Tuberculosis Programs Are Necessary to End the Epidemic." Journal of Infectious Diseases 216 (Supp17): S673-74. https://doi.org/10.1093/ infdis/jix373.

Sachdeva, Kuldeep Singh, Ashok Kumar, Puneet Dewan, Ajay Kumar, and Srinath Satyanarayana. 2012. "New Vision for Revised National Tuberculosis Control Programme ( RNTCP ): Universal Access - ‘ Reaching the Un-Reached ." Indian Journal of Med Res 135 (May): 690-94.

Salve, Solomon, Kabir Sheikh, and John D H Porter. 2016. "Private Practitioners' Perspectives on Their Involvement With the Tuberculosis Control Programme in a Southern Indian State." Int J Health Policy Manag 5 (11): 631-642. https://doi.org/10.15171/ijhpm.2016.52.

Satyanarayana, S, R Subbaraman, P Shete, G Gore, J Das, A Cattamanchi, and K Mayer. 2015. "Quality of Tuberculosis Care in India : A Systematic Review.” Int J Tuberc Lung Dis 19 (7): 751-63.

Satyanarayana, Srinath, Sreenivas Achutan Nair, Sarabjit Singh Chadha, Roopa Shivashankar, Geetanjali Sharma, Subhash Yadav, Subrat Mohanty, Vishnuvardhan Kamineni, Nevin Charles, et al. 2011. "From Where Are Tuberculosis Patients Accessing Treatment in India ? Results from a CrossSectional Community Based Survey of 30 Districts." PLOS ONE 6 (9): e24160. doi:10.1371/journal. pone.0024160. https://doi.org/10.1371/journal.pone.0024160.

Satyanarayana, Srinath, Sreenivas Achutan Nair, Sarabjit Singh Chadha, Roopa Shivashankar, Geetanjali Sharma, Subhash Yadav, Subrat Mohanty, Vishnuvardhan Kamineni, Nevin Charles Wilson, et al. 2011. "From Where Are Tuberculosis Patients Accessing Treatment in India? Results from a CrossSectional Community Based Survey of 30 Districts." PLOS ONE 6 (9). https://doi.org/10.1371/journal. pone. 0024160 .

Satyanarayana, Srinath, Sreenivas Achutan Nair, Sarabjit Singh Chadha, Roopa Shivashankar, Geetanjali Sharma, Subhash Yadav, Subrat Mohanty, et al. 2015. "From Where Are Tuberculosis Patients Accessing Treatment in India? Results from a Cross-Sectional Community Based Survey of 30 Districts" 6 (9). https://doi.org/10.1371/journal.pone.0024160.

Sharma, Surendra K, Alladi Mohan, L S Chauhan, J P Narain, P Kumar, D Behera, K S Sachdeva, et al. 2013. "Contribution of Medical Colleges to Tuberculosis Control in India under the Revised National Tuberculosis Control Programme (RNTCP): Lessons Learnt \& Challenges Ahead.” The Indian Journal of Medical Research 137 (2): 283-94.

Sheikh, Kabir, Prasanna Saligram, and Lakshmi E Prasad. 2013. "Mapping the Regulatory Architecture for Health Care Delivery in Mixed Health Systems in Low- and Middle-Income Countries."

Sheikh, Kabir, Prasanna S Saligram, and Krishna Hort. 2015. "What Explains Regulatory Failure ? Analysing the Architecture of Health Care Regulation in Two Indian States," no. December 2013: 39-55. https://doi.org/10.1093/heapol/czt095.

Simmons, Ruth, Peter Fajans, and Laura Ghiron. 2007. "Scaling up Health Service Delivery: From Pilot Innovations to Policies and Programmes." Scaling up Health Service Delivery: From Pilot Innovations to Policies and Programmes. https://doi.org/10.1016/j.puhe.2009.05.012.

Simpson, D. Dwayne. 2002. "A Conceptual Framework for Transferring Research to Practice." Journal of Substance Abuse Treatment 22 (4): 171-82. https://doi.org/10.1016/S0740-5472(02)00231-3.

Sinanovic, Edina, and Lilani Kumaranayake. 2010. "The Motivations for Participation in Public - Private Partnerships for the Provision of Tuberculosis Treatment in South Africa." Global Public Health 5 (5): 479-92. 
Sreeramareddy, C T, Z Z Qin, S Satyanarayana, R Subbaraman, and M Pai. 2014a. "Delays in Diagnosis and Treatment of Pulmonary Tuberculosis in India : A Systematic Review SUMMARY." Int J Tuberc Lung Dis 18 (3): 255-66.

. 2014b. "Delays in Diagnosis and Treatment of Pulmonary Tuberculosis in India : A Systematic Review SUMMARY." The International Journal of Tuberculosis and Lung Disease 18 (3): 255-66.

Sreeramareddy, Chandrashekhar T, Zhi Zhen Qin, Srinath Satyanarayana, Ramnath Subbaraman, Madhukar Pai, Abdul Rahman, Bandar Sungai Long, Correspondence Madhukar Pai, and Associate Professor. 2014. "Delays in Diagnosis and Treatment of Pulmonary Tuberculosis in India: A Systematic Review.” Int J Tuberc Lung Dis 18 (3): 255266. https://doi.org/10.5588/ijtld.13.0585.

STOP TB Partnership. 2017. "90(90)90, The Tuberculosis Report for Heads of State and Governments; Global Plan to End TB 2016-2020.” http://www.stoptb.org/assets/documents/resources/publications/ acsm/909090_PDF_LR.pdf.

Strauss A, Corbin J. 1997. Grounded Theory in Practice.

Subbaraman, Ramnath, Ruvandhi R. Nathavitharana, Srinath Satyanarayana, Madhukar Pai, Beena E. Thomas, Vineet K. Chadha, Kiran Rade, Soumya Swaminathan, and Kenneth H. Mayer. 2016. "The Tuberculosis Cascade of Care in India's Public Sector: A Systematic Review and Meta-Analysis." PLoS Medicine 13 (10): e1002149.doi:10.1371/journal.pmed.1002149. https://doi.org/10.1371/ journal.pmed.1002149.

Subramanian, Savitha, Joseph Naimoli, Toru Matsubayashi, and David H. Peters. 2011. "Do We Have the Right Models for Scaling up Health Services to Achieve the Millennium Development Goals?" BMC Health Services Research 11 (336). https://doi.org/10.1186/1472-6963-11-336.

Tanimura, T., E. Jaramillo, D. Weil, M. Raviglione, and K. Lonnroth. 2014. "Financial Burden for Tuberculosis Patients in Low- and Middle-Income Countries: A Systematic Review." European Respiratory Journal 43 (6): 1763-75. https://doi.org/10.1183/09031936.00193413.

TB, Central, Division Directorate, Health Services Ministry, Family Welfare, Nirman Bhavan, and New Delhi. n.d. "Module for MPWs and Other DOT Providers Module for Multi-Purpose Workers and Other DOT Providers."

Thomas, Beena Elizabeth, Banurekha Velayutham, and Kannan Thiruvengadam. 2016. "Perceptions of Private Medical Practitioners on Tuberculosis Notification : A Study From.” PLoS ONE 11 (1): e0147579. doi:10.1371/journal.pone.0147579. https://doi.org/10.1371/journal.pone.0147579.

Thomas, Beena Elizabeth, Banurekha Velayutham, Kannan Thiruvengadam, Dina Nair, Sukendu Bikas Barman, Lavanya Jayabal, Senthanro Ovung, and Soumya Swaminathan. 2016. "Perceptions of Private Medical Practitioners on Tuberculosis Notification: A Study from Chennai, South India." PLoS ONE 11 (1): 1-9. https://doi.org/10.1371/journal.pone.0147579.

Udwadia, Zarir F. 2012. "MDR, XDR, TDR Tuberculosis : Ominous Progression.” Thorax 67 (doi:10.1136/thoraxjnl-2012-201663): 286-88. https://doi.org/10.1136/thoraxjnl-2012-201663.

Udwadia, Zarir F, Lancelot M Pinto, and Mukund W Uplekar. 2010a. "Tuberculosis Management by Private Practitioners in Mumbai, India : Has Anything Changed in Two Decades ?" PLoS ONE 5 (8): e12023. doi:10.1371/journal.pone.0012023 Editor: https://doi.org/10.1371/journal.pone.0012023.

. 2010b. "Tuberculosis Management by Private Practitioners in Mumbai , India : Has Anything Changed in Two Decades ?" PloS One 5 (8): e12023. https://doi.org/10.1371/journal.pone.0012023.

. 2010c. "Tuberculosis Management by Private Practitioners in Mumbai , India : Has Anything Changed in Two Decades ?" PLOS ONE 5 (8): e12023. doi:10.1371/journal.pone.0012023. https://doi. org/10.1371/journal.pone.0012023. 
Unger, J.P., De Paepe, P., Ghilbert, etal. 2010. "PPM-DOTS Strategy for Tuberculosis Control: How Evidence-Based Is It?" International Health and Aid Policies: The Need for Alternatives, Cambridge University Press. pp. 57-66.

Uplekar, Mukund, S. Juvekar, S. Morankar, S. Rangan, P.Nunn. 1998. "Tuberculosis Patients and Practitioners in Private Clinics in India." Int J Tuberc Lung Dis 2 (4): 324-29.

Uplekar, M. 2016. "Public-Private Mix for Tuberculosis Care and Prevention . What Progress? What Prospects ?" Int J Tuberc Lung Dis 20 (11): 1424-29.

Uplekar, Mukund. 2003. "Involving Private Health Care Providers in Delivery of TB Care : Global Strategy." Tuberculosis 83: 156-64. https://doi.org/10.1016/S1472-9792(02)00073-2.

. 2013. "Scaling up of Public-Private Mix: Collaborate or Regulate." Int J Tuberc Lung Dis 17 (9): 1122.

Uplekar, Mukund, Sachin Atre, William A. Wells, Diana Weil, Rafael Lopez, Giovanni Battista Migliori, and Mario Raviglione. 2016. "Mandatory Tuberculosis Case Notification in High Tuberculosis-Incidence Countries: Policy and Practice.” European Respiratory Journal 48 (DOI: 10.1183/13993003.00956-2016): 1571-81. https://doi.org/10.1183/13993003.00956-2016.

Uplekar, Mukund, Vikram Pathania, and Mario Raviglione. 2001. "Private Practitioners and Public Health : Weak Links in Tuberculosis Control." The La 358: 912-16.

Uplekar, Mukund, Diana Weil, Knut Lonnroth, Ernesto Jaramillo, Christian Lienhardt, Hannah Monica Dias, Dennis Falzon, and Katherine Floyd. 2015. "Viewpoint WHO 's New End TB Strategy." Lancet 385: 1799-1801. https://doi.org/10.1016/S0140-6736(15)60570-0.

Veesa, Karun Sandeep, Kamalabhai Russell John, Patrick K Moonan, Puthupalayam Kaliappan, Krishna Manjunath, Karuna D Sagili, Chinnappareddy Ravichandra, et al. 2018. "Diagnostic Pathways and Direct Medical Costs Incurred by New Adult Pulmonary Tuberculosis Patients Prior to AntiTuberculosis Treatment - Tamil Nadu , India.” PloS One 13 (2): 1-14. https://doi.org/10.5061/ dryad.31k1f.Funding.

Venkat Raman, James Warner. 2008. "Public / Private Partnership in Health Care Services in India."

Verma, Dr. Madhur. 2017. "Tuberculosis Elimination in India: Bigger Goals, Smaller Commitments." Epidemiology International 2 (1): 1-2. https://doi.org/10.24321/2455.7048.201701.

Vyas, R M, P M Small, and K Deriemer. 2003. "The Private-Public Divide : Impact of Conflicting Perceptions between the Private and Public Health Care Sectors in India SUMMARY." Int J Tuberc Lung Dis 7 (6): 543-49.

Watkins, R E, K T Feeney, O Abu Bakar, and A J Plant. 2006. "Joining the DOTS in Bali : Private Practitioners 'Perceptions of Tuberculosis Control.” Int J Tuberc Lung Dis 10 (9): 988-94.

Wells, Katherine West Slevin and Anna Forbes and William. 2014. "Public Private Mix Models for the Sustainability of Successful TB Control Initiatives."

West Slevin, Katherine, Anna Forbes, and William Wells. 2014. "Public Private Mix (PPM) Models for the Sustainability of Successful TB Control Initiatives. A Working Meeting Co-Convened by USAID and the World Bank, in Collaboration with the Stop TB Partnership's PPM Subgroup, and Organized with PATH."

WHO. 2010. "Public Private Mix for TB Care and Control_A Tool Kit." WHO.

World health organisation/CDC/TB/2000.282. 2000. "Informal Consultation on Partnership with TB."

World health organisation. 1994. "Tuberculosis, a Global Emergency." https://doi.org/10.7326/0003-4819149-3-200808050-00241. 
World Health organisation. 2014. "Global Strategy and Targets for Tuberculosis Prevention, Care and Control after 2015."

World Health Organisation.. 2016. "Universal Access to TB Care."

World Health Organisation. 2016. "Report of the Joint Monitoring Mission-Revised National Tuberculosis Programme."

World Health Organization, Management Sciences for Health, The Union. 2010. "Guidance on How to Measure Contributions of Public-Private Mix to TB Control." https://www.challengetb.org/ publications/tools/ua/Guidance_on_measuring_ppm_contributions_tb_control.pdf.

World Health Organization. 2006. "Engaging All Health Care Providers. Guidance on Implementing Public-Private Mix Approaches."

World Health Organisation. 2007. "Public-Private Mix for TB Care and Control. A Tool for National Situation Assessment." Ge: World Health Organization.

World Health Organisation. 2015. "TDR | Second Round of IMPACT Grant Awardees Announced.” WHOTDR. World Health Organization. 2015.

World Health Organisation. 2016. "Global TB Report.” https://doi.org/ISBN 9789241565394.

Yellappa, Vijayashree, Neethi Rao. 2014. "Incentives for Tuberculosis Care in the Private Sector in India: A Qualitative Study.” Int J Tuberc Lung Dis 18 (11): S251. http://barcelona.worldlunghealth.org/ programme/body/Abstract_Book_2014-Web.pdf.

Yeole, R D, K Khillare, V K Chadha, T Lo, and A M V Kumar. 2015. "Tuberculosis Case Notification by Private Practitioners in Pune, India: How Well Are We Doing?” Public Health Action 5 (3): 173-79. https://doi.org/10.5588/pha.15.0031. 
